Portland State University

PDXScholar

Spring 5-24-2017

\title{
Foundational Knowledge and Other Predictors of Commitment to Trauma-Informed Care
}

Stephanie Anne Sundborg

Portland State University

Follow this and additional works at: https://pdxscholar.library.pdx.edu/open_access_etds

Part of the Psychiatric and Mental Health Commons, Public Health Commons, and the Trauma Commons

Let us know how access to this document benefits you.

\section{Recommended Citation}

Sundborg, Stephanie Anne, "Foundational Knowledge and Other Predictors of Commitment to TraumaInformed Care" (2017). Dissertations and Theses. Paper 3625.

https://doi.org/10.15760/etd.5517

This Dissertation is brought to you for free and open access. It has been accepted for inclusion in Dissertations and Theses by an authorized administrator of PDXScholar. Please contact us if we can make this document more accessible: pdxscholar@pdx.edu. 
Foundational Knowledge and Other Predictors of

Commitment to Trauma-Informed Care

by

Stephanie Anne Sundborg

A dissertation submitted in partial fulfillment of the requirements for the degree of

\author{
Doctor of Philosophy \\ in \\ Social Work and Social Research
}

Dissertation Committee:

Julie Rosenzweig, Chair

Junghee Lee

Greg Townley

Diane Yatchmenoff

Portland State University

2017 
(C) 2017 Stephanie Anne Sundborg 


\begin{abstract}
Trauma-informed care (TIC) is an approach to service delivery based on the understanding of the prevalence of psychological trauma among service users, knowledge about the impact trauma has on engagement to services, and recognition that service settings can be re-traumatizing. For more than a decade, momentum has been building on this topic. Practitioners are pursuing the knowledge and skills needed to implement trauma-informed service delivery, while organizations are building infrastructure and processes aimed at supporting this approach. Disciplines across many human service sectors are eager to incorporate TIC into policy and practice. Despite this enthusiasm, implementation efforts are slow. Acquiring foundational knowledge about TIC has typically been recommended as a first step when implementing a trauma-informed approach. However, slow progress in implementation suggests knowledge may not be enough. This study investigated the individual characteristics that impact a commitment to TIC, with specific attention to the relationship between foundational knowledge about trauma-informed care and commitment to TIC. Other variables of interest included perceived principal support, TIC self-efficacy, beliefs about trauma and its impact, and organizational strain. Survey data were collected from 118 participants working in mental health, public health, and early childhood. Results from structural equation modeling suggest that foundational knowledge predicts affective commitment to TIC both directly
\end{abstract}


and with the partially mediated paths through principal support, TIC self-efficacy, and beliefs about trauma. Organizational strain does not moderate these effects. However, group differences based on high and low levels of perceived organizational strain were observed and discussed. These findings add to the growing literature on TIC and should be considered as organizations strive to implement TIC. 


\section{Dedication}

I dedicate this study to the many service providers who recognize the need for traumainformed care and are working tirelessly to use this lens in practice and policy making. 


\section{Acknowledgements}

It does take a village, and there is no better reminder than going through a doctoral

program. With support, guidance, expertise, and love from many individuals in my life, I was able to accomplish a lofty goal. For this, I will be forever grateful.

To my committee, I appreciate your knowledge, advice, and high-quality standards. I learned a great deal from each of you and value the unique contribution you made to my success. Whether it was helping me brainstorm ideas, reviewing statistical analysis and methods (pasta and $p$ values at Olive Garden), or making sure my writing was clear, I was supported. I am especially thankful for my chair, Dr. Julie Rosenzweig, who has been by my side throughout the program. You've helped me stay focused, inspired, and moving forward. As a champion and advocate, you pushed my thinking and always helped me reach higher. Thank you.

To my colleagues and friends at Trauma Informed Oregon, a big thank you for the thinking and expertise you have shared with me over these last few years. I have learned so much about the impact of trauma and trauma-informed care during my time at TIO and look forward to watching this field flourish.

To the service providers with whom I have spent countless hours thinking about trauma-informed care implementation, I appreciate your wisdom. As a result, my own thinking is more realistic and practical. A special thank-you to La Pine Community 
Health Center, Deschutes County Health Services, and all of the home visiting professionals throughout Oregon.

To my family, I appreciate the endless support and encouragement you have given me during this process. To my Mom and Dad, you are amazing role models. With your own impressive accomplishments, you have taught me to give back and make the world a better place. I cherish the special time we had together because of my overnights in Portland. To my husband, Tom, and my children, Mackenzie, Zach, and Alex, your patience, tolerance, and belief in me made this all possible. I couldn't feel more supported. Thank you! 


\section{Table of Contents}

$\begin{array}{lr}\text { Abstract } & \text { i } \\ \text { Dedication } & \text { iii }\end{array}$

Acknowledgements $\quad$ iv

List of Tables vii

List of Figures viii

Chapter 1 - Introduction 1

$\begin{array}{lr}\text { Chapter } 2 \text { - Literature Review } & 8\end{array}$

Chapter 3 - Research Hypotheses 51

$\begin{array}{ll}\text { Chapter } 4-\text { Methods } & 57\end{array}$

$\begin{array}{ll}\text { Chapter } 5 \text { - Results } & 73\end{array}$

$\begin{array}{ll}\text { Chapter 6 - Discussion } & 102\end{array}$

$\begin{array}{ll}\text { References } & 127\end{array}$

Appendices

Appendix A. Sample Demographics $\quad 166$

Appendix B. Measures Included in the Study 168

Appendix C. Descriptive Statistics 177

Appendix D. Exploratory Factor Analysis 182 


\section{List of Tables}

Page

Table $1 \quad$ Mean Values for Specific Knowledge Topics 75

Table 2 Pearson Correlations for Variables in the Model 78

Table $3 \quad$ Component Matrix for Beliefs Scale - Fixed Factor 81

Table $4 \quad$ Component Matrix Organizational Strain - Fixed Factor $\quad 84$ Principal Components Analysis

$\begin{array}{llr}\text { Table } 5 & \text { Model Fit Indices } & 88\end{array}$

Table $6 \quad$ Comparison of Estimates using Full Information Maximum $\quad 89$ Likelihood and Mean Imputation

Table $7 \quad$ Standardized Direct and Indirect Effects between 93

Knowledge and Affective Commitment

Table $8 \quad$ Estimates of Direct and Indirect Effects of the Final Model $\quad 94$

Table 9 Interaction Effects 96

Table $10 \quad$ Standardized Estimates Compared across High 97

Table 11 Individual Path Comparisons between High Strain 99 and Low Strain Groups 


\section{List of Figures}

Page

Figure 1 Conceptual Model Illustrating the Effects of Knowledge on Affective Commitment to TIC

Figure 2 Full Hypothesized Model

Figure $3 \quad$ Direct Effects of Foundational Knowledge 53

Figure 4 Direct Effects of Perceived Principal Support, TIC

Self-Efficacy, Beliefs, and Organizational Strain on Affective Commitment

Figure $5 \quad$ Mediated Effects between Knowledge and Affective

Commitment through Self-Efficacy and Beliefs about Trauma

Figure $6 \quad$ Moderated Effects of Organizational Strain 56

Figure 7 Standard Estimates of the Hypothesized Model 86

Figure $8 \quad$ Standard Estimates of the Re-Specified Model 87

Figure 9 Indirect Paths from Foundational Knowledge to Affective 


\section{Chapter 1}

\section{Introduction}

The need for trauma-informed care (TIC) in human services is well accepted. Psychological trauma is pervasive within the population at large (see Black et al., 2011; Dube, Felitti, Dong, Giles, \& Anda, 2003; Felitti et al., 1998), but it is especially commonplace among service recipients and the workforce involved in human service systems (see Hopper, Bassuk, \& Olivet, 2010; Ko et al., 2008; Substance Abuse and Mental Health Administration [SAMHSA], 2014a). In fact, psychological trauma and related disorders are being called a global public health issue (Baker, Brown, Wilcox, Overstreet, \& Arora, 2015; Lowe, Blachman-Forshay \& Koenen, 2015). Over the past 15 years, providers have come to understand the reality that service recipients and staff can be re-traumatized by the human service system, affecting their engagement with the very services intended to provide help and healing (Bloom \& Farragher, 2013; SAMHSA, 2012, 2014a, 2014b). A trauma-informed approach takes this possibility into account, requiring that providers review services and settings in order to create welcoming, safe, and empowering environments for service recipients and staff (see Harris \& Fallot, 2001 for a seminal manuscript on this topic).

TIC is a response to three significant social problems. 
1. Trauma prevalence is high among individuals engaged with human services (Black et al., 2011; Burke, Hellman, Scott, Weems, \& Carrion, 2011; Corbin et al., 2013; Hopper et al., 2010; Ko et al., 2008; SAMHSA, 2014a).

2. Adversity, in childhood in particular, is associated with negative adult outcomes in physical health, mental health, and social functioning (Black et al., 2011; Dube et al., 2003; Felitti et al., 1998; Mersky, Topitzes, \& Reynolds, 2013; Van Niel, Pachter, Wade, Felitti, \& Stein, 2014).

3. The service system can be re-traumatizing for survivors and the workforce, impacting use and engagement (Bloom, 2010; Bloom \& Farragher, 2011, 2013; Harris \& Fallot, 2001; Hopper et al., 2010).

While early efforts focused on conceptualizing TIC and its underlying principles, attention is now directed at implementation (e.g., Miller \& Najavits, 2012; Morrissey et al., 2005). TIC is being prioritized within many at-risk populations including homeless individuals and families (Hopper et al., 2010), homeless veterans (Dinnen, Kane, \& Cook, 2014), individuals with intellectual and developmental disabilities (I/DD; Keesler, 2014), and women with mental health and other co-occurring disorders (Savage, Quiros, Dodd, \& Bonavota, 2007). Across many disciplines, service providers are acquiring the training and education needed to create trauma-informed service settings yet they struggle to transfer this knowledge, despite enthusiasm and interest (Hodgdon, Kinniburgh, Gabowitz, Blaustein, \& Spinazzola, 2013; Hopper et al., 2010). Although a few studies suggest trauma-informed policies and practices are already in use in some 
agencies (Kusmaul, Wilson, \& Nochajski, 2015; Wolf, Green, Nochajski, Mendel, \& Kusmaul, 2014), facilitating factors and barriers to implementation are not well understood (Baker et al., 2015). Instruments are available that capture progress in TIC; however, these tools are primarily aimed at organizations that have already begun implementation.

Understanding the factors impacting commitment to TIC can provide valuable insight as organizations struggle to implement a trauma-informed approach. For many organizations, adopting TIC involves making a number of changes, big and small (Trauma Informed Oregon, 2016; Yatchmenoff, Sundborg, \& Davis, in press). As organizations consider policies and practice through a trauma lens, change is inevitable. Conceptualized, according to Weiner, Amick, and Lee (2008) as any "modification in organizational composition, structure, or behavior" (p. 3), change relies on the belief that the new approach will be more effective and/or efficient than the status quo (Armenakis, Bernerth, Pitts, \& Walker, 2007; Holt, Helfrich, Hall, \& Weiner, 2010; Polites \& Karahanna, 2012).

It is well documented that change can be met with resistance (Oreg, Vakola, \& Armenakis, 2011; Self \& Schraeder, 2009), especially when the change efforts threaten our psychological sense of control, identity, and safety (Weiner et al., 2008). In fact, according to a study by McKinsey \& Company (2008), nearly two-thirds of change efforts fail and the rate is higher when change relates to organizational culture (Burnes, 2011). Decker and colleagues offered a much more discouraging success rate (only 7\%), 
suggesting that "failure in implementing change initiatives persists in organizations and is still recognized as widespread, commonplace, and costly" (2012, p. 29).

During the period preceding behavior change, organizations must attend to participation and buy-in from the individuals who will be involved in the change efforts. This commitment to change must be reflected in their willingness, attitudes, values, and beliefs. Individuals must be "primed, motivated and technically capable of executing the change" (Holt \& Vardaman, 2013, p. 9), reflecting a sense of readiness. Meyer, Srinivas, Lal, and Topolnystky (2007) described this readiness as the mindset needed to change a particular course of action, preceding both acceptance and resistance to change (Armenakis, Brown, \& Mehta, 2011). Change happens when individuals determine that a better way of conducting services is needed and that the status quo no longer meets the needs or addresses the problem. The new practice is advantageous and represents a perspective that is crucial for adoption (Dingfelder \& Mandell, 2011; Rogers, 2003).

Accounting for these individual characteristics is essential as they can be barriers to successful implementation. In fact, beliefs ${ }^{1}$ (referred to as cognitive assessments) are thought to be precursors for a change in behavior (Armenakis et al., 2007). Individual perceptions about the change can be more important than the actual change components

\footnotetext{
${ }^{1}$ In this context, beliefs is used broadly and pertains to all variables in the model (e.g., the belief that there is principal support or the belief that there is organizational strain). This is not to be confused with the specific belief about trauma construct included in the model.
} 
(Solomons \& Spross, 2011). A lack of attention to these cognitive assessments can doom an organizational change effort (Armenakis \& Harris, 2009).

The purpose of this study was to determine which factors create commitment to trauma-informed care. Foundational knowledge about the impact of trauma through training and education is central to trauma-informed service delivery, but is this enough to create commitment to TIC? Evidence is accumulating that knowledge gained as a result of training and education is correlated with changes in practice and a lack of training can be a barrier for implementation (Kramer, Sigel, Conners-Burrow, Savary \& Tempel, 2013; Layne et al., 2011). In their seminal manuscript, Harris and Fallot (2001) suggested that "with just a brief introduction to trauma dynamics, all of the personnel at a service agency can become more sensitive and less likely to frighten or re-traumatize a consumer seeking services" (p. 7). If this is the case, why is implementation so challenging for some organizations? Training and skill building may simply not be enough to prepare individuals and organizations for TIC.

The model explored in this study contained several factors hypothesized to be involved in creating commitment to TIC including self-efficacy, beliefs about trauma and its impact, and beliefs about principal support for TIC. System change efforts, often mistakenly, assume direct connections between interventions and outcomes, occurring in a linear and predictable manner. However, the reality is that systems are complicated and system change efforts must reflect the complexity of the context (Foster-Fishman, Nowell, \& Yang, 2007). Individuals vary in their perception of a situation and in their 
emotional or cognitive willingness to change (Weiner et al., 2008). In an effort to understand what works, for whom, and in what settings, the field would do well to draw from the organizational change literature.

While commitment to change is not a new consideration, identifying an explanatory model for TIC commitment is novel. Better understanding of the conditions impacting commitment to change (e.g., organizational context, organizational change process, and individual attitudes and beliefs) helps inform implementation success and failure.

A number of determinants of commitment to change have been identified in the literature. The current study focused on foundational knowledge about trauma-informed care and whether this variable explains commitment to TIC directly or is mediated by perceived principal support from one's organization, TIC self-efficacy, and beliefs about trauma (see Figure 1). Perceived organizational stress and turbulence (combined as organizational strain) was also considered as a moderator of commitment to TIC. 


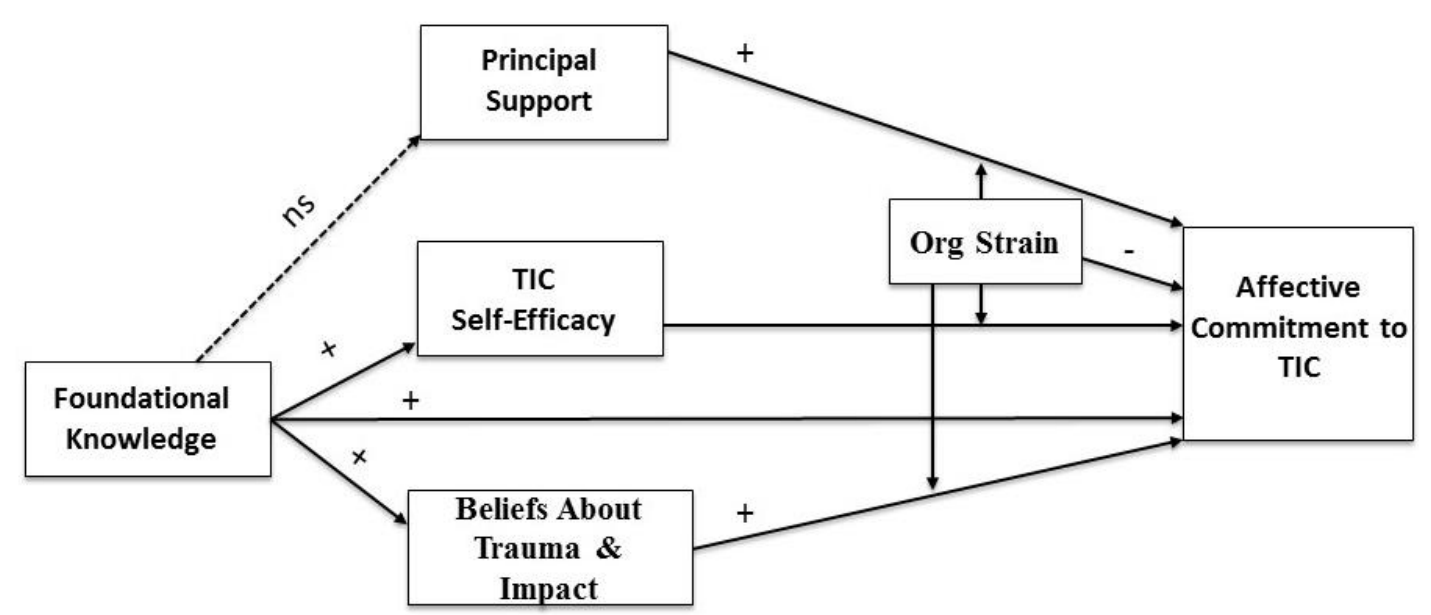

Figure 1. Conceptual model illustrating the effects of knowledge on affective commitment to TIC. Note: OrgStrain is a composite variable composed of stress and turbulence. Beliefs about trauma is a composite variable composed of 11 items. Both were treated as observed variables, see Developing Composite Variables section in Chapter 5.

Following are the questions that guided this research:

- Question 1: Is Foundational knowledge about trauma-informed care related to commitment to TIC?

- Question 2: Are beliefs about trauma, TIC self-efficacy, and perceived principal support from the organization related to commitment to TIC?

- Question 3: Do beliefs about trauma, TIC self-efficacy, and perceived principal support from the organization mediate the relationship between foundational knowledge and commitment to TIC?

- Question 4: Does organizational strain (comprised of stress and turbulence) moderate the relationship between these variables and commitment to TIC? 


\section{Chapter 2}

\section{Literature Review}

\section{Background on Trauma-informed Care}

TIC is a framework built on the understanding that service settings are highly populated with trauma survivors and that these individuals can often be re-traumatized by the actual process of service delivery (Bloom, \& Farragher, 2011; Ko et al., 2008;

SAMHSA, 2014a). To the dismay of many, TIC strategies remain unfettered and there are no fidelity measures to guide implementation. In fact, there is no universally accepted definition of TIC. Hopper, Bassuk, and Olivet (2010) noted, “Although there is agreement that 'trauma-informed' refers generally to a philosophical/cultural stance that integrates awareness and understanding of trauma, there is no consensus on a definition that clearly explains the nature of TIC" (p. 81).

That said, experts in the field agree that central to a definition is acknowledgment of the pervasiveness of trauma and recognition that trauma impacts engagement with and use of services (Bloom \& Farragher, 2011; Fallot \& Harris, 2009; Harris \& Fallot, 2001; Hopper et al., 2010). To be trauma informed, individuals or organizations, SAMHSA (2014b) has said:

Realize the widespread impact of trauma and understand potential paths for recovery; Recognize the signs and symptoms of trauma in clients, families, staff, and others involved with the system; Respond by fully integrating knowledge about trauma into 
policies, procedures, and practices; and seek to actively Resist re-traumatization. (p.

9)

History of TIC. The TIC movement began nearly 15 years ago due, in part, to the groundbreaking manuscript, Envisioning a Trauma Informed Service System: A Vital Paradigm Shift by Harris and Fallot (2001). Guided by trauma theory (Bloom, 1999, 2006; Harris \& Fallot, 2001) and the neurobiological theory of stress (Gunnar \& Quevedo, 2007; McEwen, 2008), professionals in the field had known for years that trauma impacts health and wellness. The findings from the Adverse Childhood Experiences (ACE) study (Felitti et al., 1998) supported what was intuitively known, providing compelling data to suggest a significant correlation between early adversity and poor adult outcomes including mental health, physical health, and social circumstances. Further, the Women, Co-Occurring Disorders and Violence Study (WCDVS) provided research findings about the efficacy of trauma-specific services in situations of co-morbidity with trauma, mental health, and substance use (Savage et al., 2007). The National Association of State Mental Health Program Directors (NASMHPD) and the National Technical Assistance Center (NTAC) published a Position Statement on Services and Supports to Trauma Survivors (National Association of State Mental Health Program Directors, 1998). The results of these earlier efforts became the foundation for what was to become a trauma-informed care movement (Butler \& Wolf, 2009).

Interest in and discussion about TIC implementation and guiding principles has continued to gain momentum since the early 2000s. SAMHSA held two conferences, 
Dare to Act (2004) and Dare to Transform (2008), both focusing on TIC best practices (Butler \& Wolf, 2009; Jennings, 2004). Several national organizations were created in order to further research and technical assistance for TIC including the National Child Traumatic Stress Network (NCTSN) and the National Center for Trauma Informed Care (NCTIC). Additionally, experts in the field began to offer technical assistance in behavioral health (Blanch, 2003), mental health (Jennings, 2004), child serving systems (Hodas, 2006), and organizations (Bloom, 2006).

Decades of research and scholarship have evolved into the current interest and momentum to have trauma-informed service systems (see Bloom \& Farragher, 2013; Elliott, Bjelajac, Fallot, Markoff, \& Reed, 2005; Hopper et al., 2010; Jennings, 2004; Ko et al., 2008; Wolf et al., 2014).

Principles of TIC. Harris and Fallot (2001) proposed a trauma-informed service system that includes a commitment from leadership, an approach for universal screening, ongoing training and education, trauma-informed hiring practices, and a review of policy and practice, all in order to ensure a trauma-informed focus.

Since that time, many in the field have contributed to the understanding of TIC offering principles of practice and creating toolkits and models (see Fallot \& Harris, 2006, 2009; Hopper et al., 2010; SAMHSA, 2014a, 2014b, for examples). Included in all models is attention to both physical and emotional safety (Bloom, 2006; Elliot et al., 2005; Fallot \& Harris, 2006; SAMHSA, 2014a, 2014b). This requirement is intuitive given that trauma undoubtedly challenges our sense of safety. 
The other principles included in a trauma-informed approach attend to issues of power and self-worth. Fallot and Harris (2006) suggested that a trauma-informed approach would include trust, choice, empowerment, and collaboration, in addition to safety. Elliott, Bjelajac, Fallot, Markoff, and Reed (2005) noted, “An empowerment model is essential to recovery from the overwhelming fear and helplessness that is the legacy of victimization" (p.465). Based on a synthesis of experts in the field (Community Connections, NASMHPD, NCTSN, National Center on Family Homelessness, and the Women, Co-Occurring Disorders and Violence Study), Hopper et al. (2010) expanded the Fallot and Harris list to include a need for trauma awareness and a strengths-based approach. SAMHSA (2012) added attention to culture, issues of systemic or historical oppression, and inclusion of service recipient voice. It also suggested involvement of peer support.

Overall, the values and principles may only differ in terms of semantics, as the sentiment underlying these concepts appears quite similar. For ease in application, it would be beneficial if the field could come together and pronounce a set of guiding principles.

Implementation efforts and empirical evidence. Empirical evidence regarding TIC is sparse in the literature, but the following examples of TIC implementation exemplify the focus of research thus far.

In a study (2014) investigating the use of Fallot and Harris' (2006) TIC principles (e.g., safety, trust, collaboration, empowerment, and choice), Kusmaul et al. (2015) found 
that emotional and physical safety are addressed most often, followed by empowerment. Collaboration was the least prioritized component. Among the findings, these authors identified sub-dimensions of the principles leading them to question the factorial validation of these variables. Further, they found less involvement with TIC among support staff as compared to direct service and leadership. They cautioned that this is worth disentangling as support staff have significant contact with service recipients. Finally, they found a strong correlation between implementation of these categories, suggesting that when an organization is attending to one category, it is probably attending to others. Noting sparse empirical evidence, the authors called for additional research testing these constructs and specifically testing construct validity. This study is one of the few testing the principles of trauma-informed care and they suggested that future research should use mixed methods (adding the qualitative component) to clarify findings.

Another study investigated the implementation of TIC principles (Wolf et al., 2014). Based on Fallot \& Harris (2006), this qualitative study explored whether organizations engage in trauma-informed practice, to what extent they recognize it to be trauma informed, and whether this effort is directed at staff as well as clients. Thirty-two agencies participated in focus groups and six participated in key informant interviews. Upon summarizing the qualitative findings, these authors found that most of the organizations were using these principles in practice although they might not recognize them as trauma informed. Further, many of the principles were not practiced with staff. 
A study (Hendricks, Conradi \& Wilson, 2011) assessing provider readiness for TIC using the Trauma System Readiness Tool developed by Chadwick Trauma Informed Systems Project (see www.chadwickcenter.org) found strong leadership commitment to be a facilitating factor. Among the barriers was lack of funding and lack of trauma training. These authors recommended training for all staff, structured screening, and increased cross-system collaboration.

\section{Organizational Change}

Organizational change is an "intentional effort to make differences in the organization's work setting for the purpose of enhancing individual development and improving organizational performance" (Choi, 2011, p. 2). This adaptation results from the dynamic tension between change and stability (Klarner \& Raisch, 2013). According to Appelbaum, Habashy, Malo, \& Shafiq (2012), "Successfully implementing changes can be a major determinant of an organization's short and long-term success" (p. 765). Although change is important for growth and survival, it is often met with resistance (Oreg, 2003, 2006; Oreg et al., 2011). Some individuals fear change will require the abandonment of familiar habits, while others believe change brings a loss of control. Too often change is viewed as a "fait accompli" with little recognition for change as an ongoing process (Sandberg \& Tsoukas, 2011).

Despite momentum and enthusiasm about trauma-informed care in social services, TIC requires organizational change. For many individuals and agencies, it is a paradigm shift in culture and practice and a change in mindset (Dinnen et al., 2014). Consistent 
with the findings of MacKay and Chia (2013), it reflects the changing interaction between individuals and their environment. Tsoukas and Chia (2002) offered, "Change is the reweaving of actors' webs of beliefs and habits of actions as a result of new experiences obtained through interactions" (p. 570).

As it relates to TIC, change must be understood as a process rather than a distinct state (“changed" versus "unchanged"). In fact, TIC represents a transformation, or as Langley, Smallman, Tsoukas, and Van de ven (2013) proposed, a "succession of movements of a recognizable entity over time" (p. 5). Maes and Van Hootegem (2011) described this as emergent change "when people reaccomplish routines and when they deal with contingencies, breakdowns, and other opportunities in everyday work" (p. 195). As organizations transform their character and culture, context and process are important evolutionary factors (Caldwell, Liu, Fedor, \& Herold, 2009). The deep elements of a system including attitudes, beliefs and values must be the target for transformational, second-order change both at an individual and collective level (Holt \& Vardaman, 2013; see Kuipers et al., 2014 for a review of the nature of change). Combined with experiences, expectations, and assumptions, these normative "below the surface" elements form what is considered the status quo in the organization and should be targeted in promoting readiness for system change (Foster-Fishman et al., 2007, p. 204).

\section{Process of Change}

Stages-of-change models are frequently used for guidance in research. One of the most popular frameworks is Prochaska and DiClemente's (1982) trans-theoretical model 
of change. According to this theoretical framework, individuals move through five stages of cognitive change: precontemplation, contemplation, preparation, action, and maintenance. Precontemplation is the stage when individuals gain awareness about a problem. People may remain in this stage if they are not convinced a change is needed, or if they have reasons to feel resistant to change (Prochaska, 2013). Contemplation marks an increase in willingness to change yet there are still barriers to implementation, often manifested as procrastination. Those in the preparation stage have more immediate intentions to act and it is this group that should be targeted as change leaders. Many studies of readiness for change use this model as a framework (see Barrett, Haslam, Lee, \& Ellis, 2005; Kazemi, Wagenfeld, Van Horn, Levine, \& Dmochowski, 2011 for recent examples), suggesting readiness is akin to preparation or taking action in the near future.

Another common stages-of-change framework, and one that provides a theoretical anchor for this study, is Lewin's (1951) model introducing the transformative periods of unfreezing, moving, and finally re-freezing. The period of unfreezing aligns best with readiness as staff begin to perceive the status quo as being less than optimal and perceive a new vision for the future as more desirable. During the period of unfreezing, individuals develop the confidence and readiness needed to pursue the change. This stage is important because readiness ensures that individual change recipients will be more invested in and make greater effort toward the change even in the presence of barriers (Armenakis et al., 2011; Weiner et al., 2008). Individuals vary with regard to their perception of a situation and their emotional or cognitive willingness to change (Weiner 
et al., 2008). Understanding this variability is important when identifying potential leverage points. Foster-Fishman, Nowell, and Yang (2007) note, "Depending on the readiness of a system, sometimes identifying and shifting a key lever sets the whole system in motion towards a more desirable end state" (p. 204).

In addition to the stages-of-change models described above, implementation science has provided numerous frameworks through which to view the implementation process (see reviews in Meyers, Durlak, \& Wandersman, 2012; Wisdom, Chor, Hoagwood, \& Horwitz, 2014). Rogers (2003), in describing the "diffusion of innovation" (p. 11) was among the first to describe the stages of implementation. These stages include dissemination, adoption, implementation, evaluation, and institutionalization. Similarly, Simpson (2002) developed the Organizational Readiness for Change scales (ORC) in order to outline the transfer of research to practice. In this model, he outlines four key phases in organizational change including exposure, adoption, implementation, and practice. The exposure phase aligns well with the knowledge component of the current study as organizations provide information through training and consultation in order to increase motivation for change. The next phase is also pertinent to the current discussion as adoption must take place at both the leadership and staff levels, and it must reflect both willingness and ability (Weiner et al., 2008). These recommendations are all in keeping with the investigation of commitment to TIC, and they are part of the beliefs needed in order to commit to and implement a trauma-informed approach. 
A thorough discussion of the implementation process is outside the scope of the current study; however, understanding the factors introduced in implementation models can be informative when considering commitment to change. According to Meyers, Durlak, and Wandersman (2012), "Implementation frameworks have been described as windows into the key attributes, facilitators, and challenges related to promoting implementation" (p. 465).

In a review of 25 frameworks, Meyers et al. (2012) concluded that four implementation phases are necessary for quality implementation. The first two phases occur before implementation begins and focus on infrastructure and capacity. In phase one, assessment of the situation (including need, ability, and readiness) and identification of strategies for building capacity are prioritized. During this phase, organizations will be answering questions such are "What problems or conditions will the innovation address?" "To what degree does the organization / community have the will and the means (resources, skills, and motivation)?” “Are we ready for this?' (Meyers et al., 2012, p. 469). These important considerations align well with the stage of readiness described below.

\section{Readiness for Change}

Over the past decade, an extensive literature has accumulated outlining organizational readiness for change (see Choi, 2011 for a review). Many see readiness for change as a discrete stage in the process of implementation. For example, Rogers (2003) described readiness as the persuasion stage of his diffusion of innovation model. The concern-based 
adoption model (Hall and Hord, 2006) includes the conceptualization of readiness as a way to promote a sense of concern for those affected by change. Armenakis and Harris (2009) suggested that readiness falls between diagnosis and adoption in the journey of change.

Readiness incorporates both psychological factors, captured by attitudes and beliefs, and structural factors that reflect current organizational circumstances (Bouckenooghe, 2010; Bouckenooghe, Devos, \& Van den Broeck, 2009). Of the factors involved in change readiness, change-related beliefs are considered among the most important (see Armenakis \& Harris, 2002, 2009; Armenakis, Harris, \& Mossholder, 1993 for seminal manuscripts on this topic).

Damschroder et al. (2009) suggested that agency conditions such as leadership involvement, availability of resources (e.g., training, education, time, and money), information, and other sources of knowledge building all contribute to organizational readiness and commitment to change. It is now widely recognized that individual response to change can determine its success or failure (Bartunek, Rousseau, Rudolph, \& DePalma, 2006; Kim, Hornung, \& Rousseau, 2011; Oreg et al., 2011). Holt, Armenakis, Field, and Harris (2007) noted that beliefs are comprehensive and reflect the process, content (actual change under consideration), organizational context, and individual characteristics. Assessing individual beliefs can provide a "barometer of the degree of buy-in among change recipients" (Armenakis et al., 2007, p. 499), reflecting both an individual's perceived ability and his or her willingness. 
According to the readiness for change model proposed by Armenakis, Bernerth, Pitts, and Walker (2007), change agents must believe that a new way of operating is needed (discrepancy), that the change being considered is the most suited for the situation (appropriateness - saliency), that the individual or organization has the capability to manage this change (efficacy), that the change will be supported by leadership as well as other staff within the organization (principal support), and that the change will benefit them personally (valence). An awareness of a gap between what is and what could be helps motivate change (Weiner et al., 2008). However, identifying the gap is only the first step in a change process. The proposed alternative must be viewed as acceptable.

Furthermore, individuals must believe that they are capable of such changes and that they have the support of others, including organizational leaders and peers who will support the change. These beliefs underlie a readiness for change and must be addressed and even influenced before progress can be made (Armenakis \& Harris, 2009). Incongruent beliefs and values from change agents can result in resistance to change efforts. This is especially true in terms of second-order changes aimed at transformational change (Choi, 2011; Foster-Fishman et al., 2007).

An individual who is ready to change "exhibits a proactive and positive attitude toward change, which can be translated into willingness to support change and confidence in succeeding in change" (Vakola, 2014, p. 196). That said, readiness for change is conceptualized in several ways across the literature: an individual state of 
readiness, perceived organizational readiness, and actual organizational readiness (Vakola, 2014).

Whether a change is being adopted and/or institutionalized or whether the organization is still creating readiness, change agents must "anticipate, consider, and plan to influence and shape these beliefs in pursuit of readiness for change, implementation support, and change commitment" (Armenakis et al., 2007, p.129). Beliefs represent areas of opportunity when commitment (or intent) to change seems to stall. Readiness factors are "theorized to act as levers that could overcome habitual or trained 'inertia' in the form of commitment to the status quo" (Jaros, 2010, p. 323).

Readiness for change has been included in many studies investigating the change process as a predictor variable (e.g., Neves, 2009), as a mediating variable (Drzensky, Egold, \& van Dick, 2012; Jack-Walker, Armenakis, \& Bernerth, 2007; Jones, Jimmieson, \& Griffiths, 2005), and as an outcome variable (Choi \& Ruona, 2011; Vakola, 2014). For example, Nordin (2012) found that both a transactional leadership style and organizational commitment explain readiness for change.

Despite the consideration of readiness for change across numerous disciplines, there is limited research about readiness as it relates to TIC. In one example, the trans-theoretical model (Prochaska \& DiClemente, 1982) was used in a case study concerning a residential treatment center that examined readiness across several change dimensions, including knowledge and effort toward the change, support of the change including leadership and resources, and organizational climate. Findings suggested that the residential treatment 
program's readiness was at the preparation, initiation, or stabilization stage for all dimensions involved in change (Farro, Clark, \& Hopkins-Eyles, 2011).

Another example, and one that closely aligns with the current study, explored readiness for a specific trauma-informed model using the readiness-for-change beliefs (ROC; Armenakis et al., 2007). Esaki, Hopson, and Middleton (2014) investigated the relationship between the readiness beliefs (appropriateness, efficacy, principal support, and valence) and perceived successful implementation of the Sanctuary model (Bloom, 2005). Albeit with a small sample ( $n=37)$, the authors found that perceived implementation was significantly related to belief of appropriateness and belief in principal support. Self-efficacy and valence were not related to perception of implementation. They also found among agency groups that leadership was the least likely to demonstrate the Sanctuary principles. This study differs from the current research proposal as it investigated readiness two years after the Sanctuary model had been introduced.

Readiness beliefs have been shown to predict commitment to change (Jack-Walker et al., 2007). However, less is understood about the predictors for readiness. The proposition explored in this study is that foundational knowledge will predict readiness beliefs, which in turn will predict commitment to change. The relationship between beliefs and foundational knowledge will create a state of readiness. However, the readiness state will predict commitment to TIC under low but not high organizational strain conditions. Variables included in this study are described in more detail below. 


\section{Commitment to Change}

The model proposed in this study explores the relationship between foundational knowledge, change-related beliefs about trauma and TIC, and commitment to change. Commitment to change extends readiness by incorporating the intention to change behavior (Herscovitch \& Meyer, 2002). Reflecting a "psychological alignment" to the change (Herold, Fedor, Caldwell, \& Liu, 2008, p. 347), commitment is more than simply a positive attitude toward change or the absence of resistance. Instead, commitment represents the intention to make change successful (Fedor, Caldwell, \& Herold, 2006), which has been shown to be a strong indicator of actual behavior change: "When appropriately measured, behavioral intentions [commitment] account for an appreciable proportion of variance in actual behavior" (Ajzen \& Fishbein, 2005, p. 188).

Moreover, commitment to change is distinct from commitment to an organization (Fedor et al., 2006; Herscovitch \& Meyer, 2002) and is more strongly related to support of a change effort (Choi, 2011; Meyer, Srinivas, Lal \& Topolnystsky, 2007; Shin, Taylor, $\&$ Seo, 2012). Because commitment includes intention, it is an even stronger predictor of behavior than attitude alone.

Measuring commitment to change has challenged researchers for the past several decades with some arguing that the construct is one-dimensional (see Fedor et al., 2006), and others viewing it as multi-dimensional (see Choi, 2011, for a review). In a review of the commitment to organizational change literature, Jaros (2010) stated that the field will benefit from more granularity regarding the definition of commitment to change, 
specifically, identifying the development of commitment and clarifying whether commitment represents one or more dimensions.

Herscovitch and Meyer (2002) conceptualized a three-component model (TCM) of commitment that has been the basis of many studies (see Bouckenooghe, Schwarz, \& Minbashian, 2015, for a review). This conceptualization of commitment includes affective commitment (reflecting a desire to change), normative commitment (reflecting obligation to change), and continuance commitment (reflecting a perceived cost of not changing). The authors found these factors to be distinct but related. Affective commitment for change represents a positive attitude and "buy-in" and is said to measure the emotional aspect of attitudes as they relate to commitment. Indeed, affective commitment to change taps into cooperative and championing motivation, both inclusive of emotion (see also Meyer et al., 2007). Further, both affective and normative commitments to change are associated with discretionary behaviors or those that exemplify going above and beyond (Meyer, Stanley, \& Vandenberg, 2013; Shin et al., 2012), whereas continuance commitment is only related to compliance with focal behavior specific to the change (Bouckenooghe et al., 2015).

Many studies support the notion that commitment consists of distinct factors (Bouckenooghe et al., 2015). While many studies have included all three components of commitment in their studies (see Baraldi, Kalyal, Berntson, Näswall, \& Sverke, 2010; Bouckenooghe et al., 2015; Chen \& Wang, 2007; Foster, 2010), several have focused on only one or two. With interest in cooperative behavior, Michel, Stegmaier, and Sonntag 
(2010) found that a sense of procedural justice positively relates to affective commitment to change and that this relationship is mediated by identification with the organization. Neves (2009) examined the mediating role of affective commitment with change appropriateness, change-related self-efficacy, and level of individual change. As expected, a positive and significant relationship was found between perceived change appropriateness and affective commitment. He also found a significant relationship between affective commitment and level of individual change. Surprisingly, however, self-efficacy did not significantly explain affective commitment. In a study examining affective and continuance commitment only, Neves and Caetano (2009) found that affective commitment is related to work outcomes including turnover intentions, perceived performance, and organizational citizenship behaviors. This relationship is mediated by trust in the supervisor. Continuance commitment, however, is not significantly related to any of the work-related outcomes. Finally, in a study of affective and normative commitment to a significant organizational restructuring, Shin, Taylor, and Seo (2012) noted that both types of commitment are significantly predicted by psychological resilience and organizational inducements, and the relationship is mediated by measures of social exchange and affect. Further, both of these forms of commitment are significantly related to creative and behavioral support for change.

While the Herscovitch and Meyer (2002) TCM has been well examined in the literature, there is limited literature supporting the relationship between the antecedents included in the proposed model and commitment to change. In a study with affective 
commitment (Herscovitch and Meyer, 2002) as the dependent variable, Santhidran, Chandran, and Borromeo (2013) found that leadership characteristics significantly predict commitment to change. Further, this relationship is fully mediated by change-related selfefficacy, as described in the study as change readiness. Herold, Fedor, and Caldwell (2007) also found self-efficacy to be significantly related to commitment to change. Using their own one-dimensional measure of commitment, these authors examined the effect of turbulent environments (many changes happening at once) and found that turbulence interacts with self-efficacy to impact commitment to change. Not surprisingly, the individuals showing the lowest level of commitment to change were those in highly turbulent environments and having low levels of self-efficacy. Using the same commitment-to-change measure, Herold, Fedor, Caldwell, and Liu (2008) learned that transformational leadership style is more significantly predictive of commitment to change than change-specific practices. In a study investigating a student's affective commitment to his or her major and career expectations, the authors found that careerrelated self-efficacy significantly predicted expectations and mediated the relationship between this outcome and affective commitment.

Although the literature is sparse connecting organizational support and self-efficacy directly to commitment for change, change self-efficacy has been studied in terms of readiness for change (Kwahk \& Kim, 2008; Rafferty \& Simons, 2006) and openness to change (Wanberg \& Banas, 2000), and it has been shown to be significantly predictive of both constructs. 
Understanding the determinants of commitment to TIC will fill an important gap in the literature. Although research has been done in this area, the determinants of affective commitment, in general, are not well understood (Shin et al., 2012). More specifically, as it pertains to TIC, few experts in the field had identified individual commitment as a necessary element for success, despite calling for change agents and champions (Fraser et. al., 2014; Hopper et al., 2010; SAMHSA, 2014b). For instance, SAMHSA (2014b) suggested that the steps in becoming trauma-informed include gaining commitment from leadership, identifying internal change agents, creating a work group, developing a work plan, and incorporating TIC in the strategic plan, vision, and mission. Although the SAMHSA group may expect that readiness for TIC will trickle down from leadership, nowhere in their 15-step guide to TIC implementation do they note the need for creating commitment from staff.

An exception to this comes from the National Center on Family Homelessness, which stated that preliminary work must be done in order to "set the stage" for trauma-informed care (see Guarino, Soares, Konnath, Clervil, \& Bassuk, 2009, p. 54). Creating commitment to TIC is an ongoing process. A change to trauma-informed care requires all staff, in all roles, committing to not only a change in practices but also a change in culture. According to Guarino et al. (2009), “Addressing the need for change with all staff is not a one-time conversation. Commitment to change is a process that requires ongoing discussions around what it means to be trauma-informed and what it will take to begin the transformation" (p. 55). 


\section{Determinants of Commitment to Change}

Foundational knowledge. Knowledge can be acquired in a variety of ways including formal trainings, webinars and videos, books, and workplace learning. Learning environments become embedded in the culture when organizations value competency and knowledge building from management down to line staff (Jamieson \& Gellermann, 2014). When individuals perceive their organization as having a learning environment, readiness for change is stronger (Choi, 2011).

For individuals and organizations wishing to adopt a trauma-informed approach, formal training is often recommended as the first step (Fallot \& Harris, 2009; Guarino et al., 2009; Hopper et al., 2010; Lebel \& Kelly, 2014; SAMHSA, 2014b). Creating a highly skilled workforce is a priority in many fields including mental health (Fricchione et al., 2012) and public health (Hilliard \& Boulton, 2012).

Not only is knowledge about trauma and its impact necessary in order to adopt a trauma-informed approach, expert knowledge is a common characteristic among champions for change (Taylor, Cocklin, \& Brown, 2012). In a study investigating the introduction of a clinical innovation in the Department of Veteran Affairs, VanDeusenLukas et al. (2008) found that team knowledge and skills were among the most important predictors of a successful change effort. Knowledge impacted implementation, but it also influenced the perception that a change was needed and appropriate.

Many researchers discuss knowledge and training as if they are interchangeable. For instance, according to Wolf, Green, Nochajski, Mendel, and Kusmaul (2014), "For 
trauma-informed services to have the desired positive impact on clients, all staff need to receive training to increase their understanding of how direct or indirect exposure to traumatic events may impact their lives and the lives of clients" (p. 112). Focusing on training as a predictor for trauma-informed change would be in keeping with recommendations in the literature (Fallot \& Harris, 2009; Hopper et al., 2010; Lebel \& Kelly, 2014; SAMHSA, 2014). However, a training variable poses several problems. First, as noted, there are multiple methods for disseminating information about trauma, the impact of trauma, and TIC. Controlling for this variability would be extremely difficult limiting the generalizability of the findings. Second, understanding training and the resultant gain in knowledge is complicated, given the many factors that impact training effectiveness, including individual characteristics, the environmental context within which the training is offered, and the training characteristics. Training varies in type, quality, and impact, all of which makes measuring this construct difficult (Herschell, Kolko, Baumann, \& Davis, 2010). Finally, the empirical evidence exploring the effectiveness of training is mixed, creating confusion in the literature (Fairburn \& Cooper, 2011). The following discussion highlights the difficulty in using this construct in the current study despite an acknowledgment that training is an important strategy in many change efforts.

Training as a method for acquiring knowledge. Training is one of the first steps in the change process, as it builds awareness about an issue and the need for change (Damschroder \& Hagedorn, 2011; VanDeusen-Lukas et al., 2008). According to Taylor, 
Cocklin, and Brown (2012), training is an effective strategy in building influential change agents. Greenwald and colleagues (2008) found that a trauma-related training module increased the positive attitudes and understanding toward challenging clients. Appelbaum et al. (2012) found that training and skill building created a sense of empowerment for staff, which in turn created a positive impact on attitudes about change.

Training supports the application of knowledge and skills, known as training transfer. Baer et al. (2009) found among community mental health counselors that both a two-day workshop and a context-tailored training resulted in skill improvement, with little difference between the two at a four-month follow-up. Dimeff and colleagues (2009) compared online training with a trainer-led workshop. They found the online training led to greater gains in knowledge while the workshop led to increased self-efficacy and demonstrated skills. Oordt, Jobes, Fonseca, and Schmidt (2009) noted that confidence and intent to change were found at a six-month follow-up to training. In one of the few examples examining TIC training results, Kramer, Sigel, Conners-Burrow, Savary, and Tempel (2013) found that manager and supervisor knowledge increased substantially after a two-day training on trauma. In a pre/post format, participants were asked about their knowledge of the impact of trauma and utilization of trauma-informed practices. The change in trauma-informed knowledge was significant, $\mathrm{t}(81)=-16.82, \mathrm{p}<.001$, persisting at least three months post-training. Kramer and colleagues planned to follow up on the lasting impact of knowledge as well as changes in behavior as a result.

Despite these examples, empirical support of this transfer is sparse in the literature and 
skill acquisition is often "assumed rather than confirmed" (Herschell et al., 2010, p.450). In a review of 50 studies examining the effectiveness of various training methods, Herschel et al. (2010) found that behavior is unlikely to change as a result of workshop trainings, suggesting, "Most often, workshop attendance resulted in increased knowledge, but not significant changes in attitude, application of knowledge, or clinical skills" (p. 457). When skills do improve, they often decrease overtime (Moyers et al., 2008).

Evaluating training is challenging given the variation in training delivery methods. Certain methods are associated with more effective training outcomes. For instance, when training clinicians about evidence-based practices, studies show greater adherence to fidelity measures when providers have coaching in addition to training (Sholomskas, et al., 2005; Stirman et al., 2013). Train-the-trainer models are also frequently used; however, this approach can have limitations. In a study of motivational interviewing using a train-the-trainer model, Martino et al. (2011) showed that training effects diminished quickly. They believed this may have been due to workload and the fact that trainers were expected to offer training in addition to their existing tasks. Furthermore, an initial finding that the train-the-trainer model was superior to the self-study approach did not persist long-term, and the difference between the two approaches was found to be insignificant.

Although training is a commonly recommended strategy for TIC, knowledge is often the desired end result. In the current study, knowledge is more straightforward and is 
included as the independent variable. Following is a discussion of knowledge and considerations when including this variable in research.

Understanding knowledge. A thorough discussion of knowledge is beyond the scope of this current study; however, a brief overview can offer useful information in understanding how this construct will be conceptualized in this study.

Knowledge can be understood in terms of type - what is known - and quality — how well the information is understood (Glasser, Langer, \& Weber, 2013). Across disciplines (e.g., psychology or education), knowledge is characterized in many ways. It may be described as conceptual consisting of principles and definitions or procedural consisting of action sequences (Glasser et al., 2013). It may be described as explicit and declarative when it can be stated aloud (Baartman \& Bruijn, 2011) or implicit or tacit when the knowledge is processed internally and automatically. As we develop and gain experience, our brains prioritize automatic processing and tacit knowledge (Brainerd \& Reyna, 2015). In fact, we increasingly rely on the use of heuristics (cognitive shortcuts) and biases, forming "meaning based memory representations," known as gist, in order to make decisions and form judgments (Reyna, 2012, p.3). A better understanding of this type of processing and memory is helpful as it may be more closely representative of what can be retained following TIC-specific trainings.

The understanding of gist memory and representation results from an extensive literature spanning more than two decades (see Reyna \& Brainerd, 1990, for seminal literature on this topic). Understood by the fuzzy trace theory (Brainerd \& Reyna, 1990), 
gist memory (or representation) reflects the essence or essential meaning of the information (Reyna, 2012). Drawing from the field of psycholinguistics, verbatim refers to the exact wording whereas gist refers to the essence. These types of memory are different as people "encode, store, retrieve, and forget verbatim and gist memories separately and roughly in parallel" (Reyna, 2012, p. 3). While gist differs in terms of detail, it incorporates both intuition and attitude (Reyna, 2008). Adults increasingly rely on gist memory especially in cases of information overload (Fukukura, Ferguson, \& Fujita, 2013). Additionally, as information becomes more distant in time and space, verbatim memory deteriorates more quickly while gist memory is more likely to remain. Fukukura, Ferguson, and Fujita (2013) suggested, "Accessibility of gist has been shown to increase with the passing of time" (p. 664). Further, it is more likely that gist memory influences the content of verbatim memory rather than the reverse (Lloyd \& Reyna, 2009).

The challenge in considering knowledge as a predictor of a particular outcome lies in determining which type of knowledge to capture and how it can be measured. TIC is a very broad topic composed of precise and technical information as well as imprecise conceptual information. While some of the information is conducive to tests of knowledge, much of it lacks the absoluteness to be considered. Further, the full menu of TIC-related content is fairly well identified (see SAMHSA, 2012, 2014b); however, it remains an empirical question as to which knowledge is essential for a trauma-informed 
approach. Addressing this question is not a focus of this study. To that end, amount of knowledge is assessed here across a number of foundational TIC-related topics.

In addition to SAMHSA (2012, 2014b), two documents provided guidance in identifying topics and information worthy of inclusion in an assessment of knowledge. First, Advanced Social Work Practice in Trauma from the Council on Social Work Education (CSWE; 2012), outlines the knowledge and practice behaviors needed when working with traumatized clients and families. Built on and aligned with the core competencies specified by the Educational Policy and Accreditation Standards (EPAS; CSWE, 2008, 2015), this document provides a comprehensive and relevant guidepost for considering essential TIC knowledge. Another source was the Standards of Practice developed by Trauma Informed Oregon ${ }^{2}$. In this document, eight core content areas were identified as being important for workforce development. The six included in the knowledge assessment are as follows:

- Principles and implementation of trauma-informed care

- The neurobiology and impact of trauma

- The Adverse Childhood Experiences (ACE) study

- Trauma in the workforce including vicarious trauma, secondary traumatic stress, and compassion fatigue

${ }^{2}$ Standards of Practice for Trauma Informed Care can be found on the Trauma Informed Oregon website or by using this link http://traumainformedoregon.org/wp-content/uploads/2014/10/Standards-ofPractice-for-Trauma-Informed-Care.pdf) 
- Issues of power and oppression related to the experience of trauma

- Historical oppression; intergenerational trauma

Understanding an individual's knowledge about the principles and implementation of TIC is essential to the current study. In addition, understanding the neurobiology of trauma is important as this can provide an explanation for brain processing and behavior (see Gunnar \& Quevedo, 2007). An overview of the ACE Study (Felitti et al., 1998) is also necessary to increase awareness about the widespread prevalence and public health implications of trauma. SAMHSA (2014a, 2014b) suggested that awareness of the impact of historical oppression and intergenerational transmission is important as well as understanding the role and benefit of individuals with lived experience of trauma. Two important topics, that incorporate a level of opinion, are not assessed by the knowledge variable but will be measured in the beliefs about trauma variable. These include an understanding of how behaviors can reflect coping patterns established by trauma survivors and how the service setting can be re-traumatizing for survivors.

Competence, the integration of knowledge, skills, and attitudes (Baartman \& Bruijn, 2011), is also important as TIC is adopted into practice. In the current study, knowledge reflects concepts related to the nature of trauma, the impact of trauma, and principles of trauma-informed care. Self-efficacy is included in the model as a way to measure perceived competence.

It is hypothesized by this researcher, that knowledge will relate directly to a commitment to TIC, beliefs about trauma, and TIC related self-efficacy. That said, it 
remains unclear whether individuals need to have factual knowledge or whether they can simply have the gist. For example, must one remember that the amygdala is the brain structure involved in processing fear or is it enough to know that the brain detects this type of threatening stimuli and subsequently initiates a stress response?

Measuring knowledge. Understanding learning and knowledge translation is the topic of an extensive literature and robust debate (see Greenhalgh \& Wieringa, 2011 for a review). Bloom's Taxonomy (Bloom, EnglehartFurst, Hill, \& Krathwohl, 1956) is often used as a hierarchy for understanding the stages of learning, beginning with knowledge and comprehension (or the simple recall of information) and progressing to application, analysis, synthesis, and evaluation (see Krathwohl, 2002, for an overview). Knowledge and comprehension are the least developed stages as they reflect rote awareness of the facts. It isn't until we progress to application and analysis that we use, process, and apply the information (Momsen, Long, Wyse, \& Ebert-May, 2010). In many situations, this can be too difficult and time-consuming to measure (McAllister \& Guidice, 2012). According to Tarrant and Ware (2012), "Without appropriate training, most novice item writers will develop low-quality test items that test only factual recall or trivial content (p. 4). In fact, in a review of nursing textbooks, $72 \%$ assessed knowledge and comprehension only (Tarrant \& Ware, 2012).

Among the most widely used methods for assessing knowledge are multiple choice (MC) and true or false (T/F) tests. They are efficient to prepare and efficient to complete (McAllister \& Guidice, 2012), an important consideration for busy professionals. 
Two important considerations when designing tests of knowledge are discrimination and difficulty (Boland, Lester, \& Williams, 2010). Discrimination refers to the ability to differentiate between those with the knowledge and those without. Difficulty reflects the likelihood that those with the knowledge will get the answer correct, and it impacts discrimination. In other words, how difficult do the questions need to be in order to discriminate between levels of knowledge? Tasdemir (2010) found no difference between T/F tests and MC in terms of discrimination and difficulty. Further, it has been argued that with good distractors (the incorrect choices on a multiple-choice test) and wellworded T/F questions, higher levels of cognitive processing can be assessed (Campbell, 2011; Gierl \& Haladyna, 2012; Haladyna \& Rodriguez, 2013). MC tests specifically are useful in introducing topics or assessing general knowledge following training (Luiselli, Bass, \& Whitcomb, 2010).

These tests are not without controversy, however, and many believe they are unreliable and flawed (Burton, 2005, Case \& Swanson, 2002). In fact, Momsen et al., 2010 argued that both MC and T/F measure only the recognition of information. In particular, it has been suggested that T/F questions can stump those who know the information due to the tricky wording embedded in the question (McAllister \& Guidice, 2012). Others noted that guessing correctly can result in unprepared students doing well on MC and T/F tests (DiBattista, \& Kurzawa, 2011). Moreover, T/F questions can lead to false knowledge as individuals remember incorrect information provided in the test question (McAllister \& Guidice, 2012; Roediger \& Butler, 2011). 
Another limitation pertains to content. Although there is some guidance, as stated above, to the topic areas that should be included, the abundance of information from which to choose testable content is overwhelming. The field has not specified which information is essential to a trauma-informed approach; therefore, selecting questions that are both meaningful for assessment yet manageable in number (length of test) is extremely difficult. A suitable alternative is to ask about knowledge more generally. As noted, gist memory is reliable and long-lasting, and may be sufficient in order to show TIC commitment.

Self-reports of knowledge are also a common method of assessment given the difficulty administering factual tests (Brossard \& Shanahan, 2006). These, too, are not without challenges, including an under- or overestimation of knowledge (Sitzmann, Ely, Brown, \& Bauer, 2010). Kerby, Brand, Elledge, Johnson, and Magas (2005) found a weak correlation between public health practitioners' self-report of knowledge and the results of a formal knowledge assessment $(\mathrm{r}=.18)$. In order to address these limitations, the knowledge assessment was piloted as a first step in this study (see Methods for further information).

Beliefs about trauma and its impact. Scholars involved in social science have investigated the relationship among beliefs, attitudes, and behavior for many years (see Glasman \& Albarracín, 2006, for a meta-analysis). According to Merriam Webster, beliefs represent an opinion or conviction about someone or something, and attitudes represent a "predisposition to respond positively or negatively toward things, people, 
places, events, and ideas" (Bayraktar, 2011, p. 84). Beliefs influence attitudes and both influence behavior (Ajzen, 2011).

The beliefs contributing to attitudes can be either explicit or implicit, resting underneath subconscious awareness (Hahn, Judd, Hirsh, \& Blair, 2014). It is often implicit beliefs that researchers are attempting to access as these are thought to predict behavior automatically unless conscious control is exerted, for instance, in response to social desirability (Ajzen \& Fishbein, 2005). According to Rafferty and Simons (2006), "Creating readiness for change involves changing [an] individual's beliefs or the way that people think" (p. 327). Negative beliefs and attitudes have been shown within some professions in relationship to service recipient behavior (e.g., using psychiatric medication as in Phokeo, Sproule, \& Raman-Wilms, 2004) or service recipient health conditions (Brown, 2006).

Beliefs about trauma and its impact are not well represented in the literature with two areas of exception. Within the workforce serving trauma survivors, a change in beliefs, worldview, and attitudes among providers is not unusual and is well documented (Kulkarni, Bell, Hartman, \& Herman-Smith, 2013; Michalopoulos \& Aparicio, 2012; Newell \& MacNeil, 2010). These common conditions are described as burnout, vicarious traumatization, secondary traumatic stress, or compassion fatigue (Newell \& MacNeil, 2010). Vicarious trauma, by its very definition, results in a change in beliefs (Bride, Radey, \& Figley, 2007). Scholars have long argued that vicarious trauma (as a result of working with trauma survivors) can result in a change of worldview and meaning making 
(see McCann and Pearlman, 1990, for seminal literature on this topic). Specifically, an individual's "affect tolerance" or "beliefs about self and others" can change over time (Bride et al., 2007, p. 155). These authors added that social workers can develop secondary traumatic stress (STS), which results in intrusive thoughts similar to posttraumatic stress disorder (PTSD). Often this change in belief leads to increased helplessness and hopelessness (Bride et al., 2007).

The other area introducing negative beliefs about trauma is found in the literature calling for trauma education for professionals. Courtois and Gold (2009) noted insensitivity in some professionals working with trauma survivors that they attributed to a lack of education on the topic. "Professionals without knowledge of trauma or sensitization to it or its consequences may experience strong negative and horrified reactions that can impact their ability to respond positively to their patients" (Courtois \& Gold, 2009, p. 12). Courtois (2002) recognized that some causes for insensitivity among professionals included "ignorance, lack of knowledge and training about how to approach work with traumatized individuals, and personal dislike of victims and other forms of bias" reflecting individual beliefs or widespread societal attitudes (p. 40). She called for increased curricula related to trauma in high school through higher education.

Belief there is principal support for the change. The need for change can be externally motivated, coming from sources outside the organization (e.g., the national momentum for TIC) or internally motivated (Gondo, Patterson, \& Palacios, 2013). Leadership often creates the sense of need as it outlines a vision for the future and "sells" 
the change to others within the organization (Armenakis \& Harris, 2009; Oreg et al., 2011).

The process by which organizations implement change is a powerful predictor of individual commitment to the change (Choi \& Ruona, 2011; Fedor et al., 2006; Oreg et al., 2011). Specifically, resources and support from management are significantly associated with successful change implementation (VanDeusen-Lukas et al., 2008). Liu, Caldwell, Fedor, and Herold (2012) noted that perceived management support is positively associated with an individual's judgments of fairness regarding the process. They found that "support provided by management during change could enhance employees' positive view toward change-related policy and structural factors" (Liu, Caldwell, Fedor, \& Herold, 2012, p. 454).

In a study examining change characteristics (e.g., frequency of change, planning for change, and nature of change), Rafferty and Griffin (2006) found that supportive leadership is related to all three conditions. They added, "Employees with supportive leaders reported less transformational change, less frequent changes, and more planned changes" (p. 1159). They also reported that supportive leadership (in regard to change) helps reduce change-related uncertainty.

In a study investigating commitment to change, characteristics that create a desire to follow a transformational leader include a sense of trust, identification with the leader, and feeling cared for (Herold et al., 2008). Devos, Buelens, and Bouckenooghe (2007) also found that trust in leadership was related to openness to change. Transformational 
leaders get buy-in despite the absence of change-related behavior, most likely reflecting trust. Those authors found that leaders with greater transformational qualities were more likely to cultivate a commitment to change, especially in cases where impact on an individual's job was minimal. Surprisingly, actual behavior from change leaders was not related to commitment to change. With regard to TIC implementation, leadership commitment and support are essential ingredients as these individuals can most easily make changes to policy, practice, and priorities (Fallot \& Harris, 2009; Harris \& Fallot, 2001, SAMHSA, 2014b).

Belief in one's ability to change (change self-efficacy). Self-efficacy is a wellstudied construct, first appearing nearly 30 years ago in Self Efficacy: Toward a Unifying Theory of Behavioral Change (Bandura, 1977). Since that time, this influential article has been cited more than 55,281 times, according to Google Scholar, and this variable has been included in numerous studies. Related to the "conviction that one can successfully execute the behavior required to produce the outcome[s]" (Bandura, 1977, p. 193), selfefficacy is situated within social cognitive theory (see Bandura 1986, 1997, 2012). This theoretical framework is important when considering readiness for TIC because it acknowledges the interaction of both the individual and environmental conditions (Bandura, 2012). According to Bandura (2012), "Human functioning is a product of the interplay of intrapersonal influences, the behavior individuals engage in, and the environmental forces that impinge upon them" (p. 11).

Self-efficacy is central to many models and theories about organizational change and 
has become a reliable predictor in the health and education literature (see, for example, Byrne, Barry, \& Petry, 2012 [weight loss]; Beauchamp, Rhodes, Kreutzer, \& Rupert, 2011 [exercise practices]; Alivernini \& Lucidi, 2011 [school-related behaviors]).

Change-related self-efficacy is the individual belief that we can successfully accomplish the requirements of a change effort. Creating readiness first begins by creating a belief in the need for change and then building a sense of self-efficacy in terms of carrying out the actions necessary for change (Armenakis et al., 2011).

Among the examples in the organizational literature, self-efficacy has been shown to predict change-related coping (Gibbons, Dempster, \& Moutray, 2011), occupational commitment (Canrinus, Helms-Lorenz, Beijaard, Buitink, \& Hofman, 2012), readiness to change (Holt \& Vardaman, 2013; Vakola, 2014), and commitment to change (Herold, Fedor, \& Caldwell, 2007). Vakola (2014) noted, "Employees who are confident about their abilities and are able to cope with stressful events, such as change, tend to perceive change as positive and as a result they experience high levels of readiness for change" ( $\mathrm{p}$. 203). In a study investigating whether organizational context and beliefs about selfefficacy impact commitment to change, it was found that self-efficacy buffers the effects of organizational turbulence (defined as the amount of internal change happening at once), thus maintaining a commitment to change (Herold et al., 2007).

\section{Organizational Strain}

A turbulent and stressed environment is not conducive to organizational change (Lehman, Simpson, Knight, \& Flynn, 2011). Workforce stress and trauma negatively 
affect job satisfaction, the commitment to innovation, and the adoption of new ideas (Kulkarni et al., 2013). Organizational climate influences the social context including attitudes, beliefs, norms, and values (Glisson, 2007).

The perception of being overloaded and emotionally exhausted at work is a tremendous source of stress (Kulkarni et al., 2013; Mollart, Skinner, Newing, \& Foureur, 2013). In a study on the adoption of cognitive therapy among clinicians, workload (excessive paperwork) and productivity requirements were found to be the most predictive barriers to implementation (Stirman et al, 2013). Farro, Clark, and HopkinsEyles (2011) determined that perception of available resources created a lower sense of readiness despite an understanding of and ability to deliver trauma-informed services. This affected change recipients' overall commitment to TIC.

As noted earlier, Herold et al. (2007) examined the effect of turbulent environments and found that turbulence interacts with self-efficacy to impact our commitment to change. Not surprisingly, the individuals showing the lowest level of commitment to change were those with highly turbulent environments and low levels of self-efficacy.

The impact of organizational stress as it relates to trauma-informed care is also fairly well conceptualized. Organizations become stressed when the workforce is stressed, both becoming dysfunctional. Specifically, when a workforce experiences work-related trauma as a result of the populations they serve and the work they do, they start to mirror the same behaviors as the service recipients, referred to as parallel process (Bloom, 2006, 2010a, 2010b, 2011). When an agency is experiencing this internal toxicity, the 
organization is said to be trauma-organized, with characteristics including lack of emotional management, increased authoritarianism, inability to make decisions, dissociation, impaired relationships, disempowerment and helplessness, increased aggression, and unresolved grief. According to Bloom (2010), “Chronically stressed organizations tend to have significantly lowered abilities for creative change" (p. 11).

The concept of organizational stress and parallel process has made a significant contribution to the conceptualization of trauma-informed care and the barriers to traumainformed service delivery. The current study investigated whether organizational stress is also a barrier to commitment to TIC.

\section{Current TIC Assessment Instruments}

As interest in trauma-informed care has taken hold across many disciplines, practitioners and organizations are asking for implementation examples and strategies (Yatchmenoff et al., in press). As a result, many assessment instruments have been created to help them consider TIC implementation within specific domains. Many of these instruments assess progress for organizations that are already engaged, to some degree, in TIC implementation.

Through Community Connections and the work of Harris and Fallot (2001), the Trauma Informed Program Self-Assessment Scale was created, one of the first instruments to assess trauma-informed care in service settings. Following their groundbreaking manuscript, which introduced the idea that service settings could be a source of trauma for survivors engaged in services, they created this instrument as a way to assess current 
practices and intentions toward implementation, evaluation, and monitoring (Fallot \& Harris, 2006). The five values (safety, trustworthiness, empowerment, choice, and collaboration) outlined in their manuscript, Using Trauma Theory to Design Service Systems (Harris \& Fallot, 2001), provide a framework for evaluation. These values are considered across six organizational domains: the program setting, program activities, policy, leadership support, staff training and education, and hiring practices. This comprehensive assessment involves case file review, review of agency records, interviews, and focus groups. According to Farro et al. (2011), "This instrument provides a gold standard assessment that has defined trauma-informed care and provided one of the first in-depth assessments of trauma-informed practices" (p. 230).

The assessment was revised in 2009 and a planning protocol was added, Cultures of Trauma-Informed Care Program Self-Assessment Scale and a Self-Assessment and Planning Protocol (Fallot \& Harris, 2009). This document provides a brief overview of the impact of trauma as an explanation for the need for trauma-informed services. Then it discusses how the principles of TIC (safety, trustworthiness, choice, collaboration, and empowerment) can be integrated into the culture, creating the conditions necessary to take hold. The steps outlined include a planning phase, which involves cultivating leadership support and internal champions, a kickoff event that incorporates training all staff, and implementation, which is based on the self-assessment and planning protocol (scale). Evaluating change at both the service level and administration-system level, the scale provides questions addressing each of the principles, for both service recipients and staff. 
Screening, assessment, and knowledge of trauma-specific services are included in the section for staff. Across the administrative level, questions pertain to staff training and support including hiring practices.

The work (described above) from Community Connections provides a springboard for other local and state entities considering TIC implementation. For instance, in Oregon, Trauma Informed Oregon (a state initiative created as a resource for information, training, and statewide connections) created the aforementioned Standards of Practice for Trauma Informed Care that provide a thorough framework for agencies as they strive to become more trauma informed.

In 2014, the Institute for Health and Recovery developed a toolkit based on the work of the WELL (Women Embracing Life and Living) project, one of the Women, CoOccurring Disorders and Violence (WCDV) projects funded through SAMHSA. The tool kit, Developing Trauma-Informed Organizations, is based on a relationship-oriented model of change (see Markoff, Finkelstein, Kammere, Kreiner, \& Prost, 2005) and provides a very thorough roadmap for organizations wishing to incorporate a traumainformed approach. Included in the toolkit is an overview of TIC principles, a strategic planning template, examples of trauma policies, self-assessment instruments for both providers and the organization, a provider practice survey, and a supervision guide (Institute for Health and Recovery, 2014). These tools assume at least a foundational understanding of trauma and its impact as the questions are intended to measure current knowledge and practice. These instruments are well suited for agencies that already have 
staff buy-in, as there are no questions tailored to the barriers of implementation. For instance, how are staff beliefs and attitudes reflected during TIC implementation?

The National Center on Family Homelessness created a thorough assessment process geared toward homeless services. This 87 -item instrument focuses on issues of staff development, environment and setting, services, client assessment and other activities, service recipient involvement, and agency policies (Guarino et al., 2009). It is well suited for individuals who have a foundational understanding of trauma and its impact, and results can indicate whether an agency is minimally, somewhat, or highly trauma informed (with regard to policies and practice). It, too, focuses primarily on the principles of TIC and assessing whether those are adequately addressed in trauma-related practices. One of the most helpful sections of the 100-page document is a guide on creating change within the organization, which outlines the process from setting the stage to implementing the self-assessment instrument and using the results to inform an implementation plan for trauma-informed care changes. Laying the groundwork is an important step in TIC implementation, and these authors suggested gauging readiness. When staff are not ready for TIC implementation, it is necessary to provide additional education and awareness building. Guarino et al. (2009) noted, "Becoming trauma informed is as much about changing a program's culture as it is about changing program practices" (p. 69). Attitudes and beliefs can be deeply embedded within individuals, making them difficult to change. While this document notes the importance of individual attitudes and beliefs as well as the collective impact on culture, this self-assessment instrument does not attempt to measure 
existing attitudes, values, or beliefs.

Another instrument, the Trauma Informed System Change Instrument, created by the Southwest Michigan Children's Trauma Assessment Center (see Richardson, Coryn, Henry, Black-Pond, \& Unrau, 2012) takes a system-level approach to measuring change resulting from community efforts. Specifically, this work focuses on the child welfare system and better understanding TIC change resulting from community training and education building. This short (19-item) survey considers agency policy, practice, and connections from both an individual and a community perspective. The developers of this instrument understand the importance of individual characteristics and draw from the Evidence-Based Practice Attitude Scale (EBPAS; Aarons, 2004), especially with regard to openness to change. For example, "I feel favorable in trying a new trauma informed intervention with children and families", "I am willing to try a new form of intervention even if I have to follow a manual or protocol, and "I am willing to use trauma informed interventions that researchers say are effective" are among the questions that load onto a factor these authors titled openness. There is also a question that likely taps into selfefficacy as it states "I feel equipped to help children make meaning of their trauma history and current experiences from a trauma perspective." Although this instrument provides a few questions relevant to understanding the change process, it doesn't address individual beliefs about whether TIC is needed, appropriate, personally beneficial, or supported by the organization and leadership.

Also, coming from the field of child welfare, Hendricks, Conradi, and Wilson (2011) 
outlined the collaborative work of the Child Welfare Committee of the National Child Traumatic Stress Network (NCTSN) in partnership with the California Social Work Education Center, which resulted in the development of the Trauma System Readiness Tool. The intent of this instrument is to assess trauma-related knowledge and practices based on the essential elements of being trauma informed in child welfare. This 150-item assessment considers change at the individual, agency, and cross-system levels with regard to foundational knowledge, agency activities including screening and referral, and support for families and staff engaged in the child welfare system.

The field of mental and behavioral health has generated tremendous thinking and guidance about the need for and implementation of trauma-informed care. The work of SAMHSA (2012, 2014a, 2014b) continues to provide valuable resources (materials and funding) to further this field. As one example of measuring trauma-informed care within a behavioral health setting, Farro et al. (2011) conducted a readiness assessment in a behavioral health organization. The Trauma Informed Services Self-Assessment Scale and Planning Protocol (Fallot \& Harris, 2009) was used as a framework for that study as it provides "concretely defined and high standards" for trauma-informed care implementation (Farro et al., 2011, p. 230). In keeping with the concepts put forth by Fallot and Harris (2009), that assessment looked across program policies, settings, and activities to consider issues of safety, power, and value. With the aim of creating an assessment approach that can both evaluate and advance trauma-informed efforts in a behavioral health setting, the protocol combined the ACE questionnaire (see Felitti et al., 
1998); the Consumer Perception of Care instrument (Clark et al., 2008) and the Community Readiness Model (Edwards, Jumper-Thurman, Plested, Oetting, \& Swanson, 2000). Readiness for change is an important precursor to TIC implementation and this perspective is included in the protocol. Specifically, the community readiness model considers various points of readiness including awareness, resistance, planning, preparation, stabilization, expansion, and level of ownership, an extension of Prochaska and DiClemente's (1982) trans theoretical framework for change (e.g., precontemplation, contemplation, preparation, action, and maintenance). While this pilot furthered the field's awareness about the importance of understanding readiness for change, it addressed only a few of the variables. It included knowledge and leadership support; however, it failed to measure the individual beliefs that underlie buy-in. 


\section{Chapter 3}

\section{Research Hypotheses}

The aim of this study was to investigate the direct and indirect effects associated with foundational knowledge about trauma-informed care and affective commitment to TIC. Specifically, does this variable explain commitment to TIC directly, or instead, is this relationship mediated by an individual's beliefs about trauma and its impact and his or her sense of TIC-related self-efficacy? Further, how do perceived principal support (the influence of leaders and change agents) and organizational strain affect the relationships among the other variables in the model? Below are the hypotheses that guided this study.

\section{Full Model}

Hypothesis 1. A model representing all direct effects, indirect effects, and moderated effects will be a good fit for the data and will explain a significant amount of variance in affective commitment to TIC (Figure 2). 


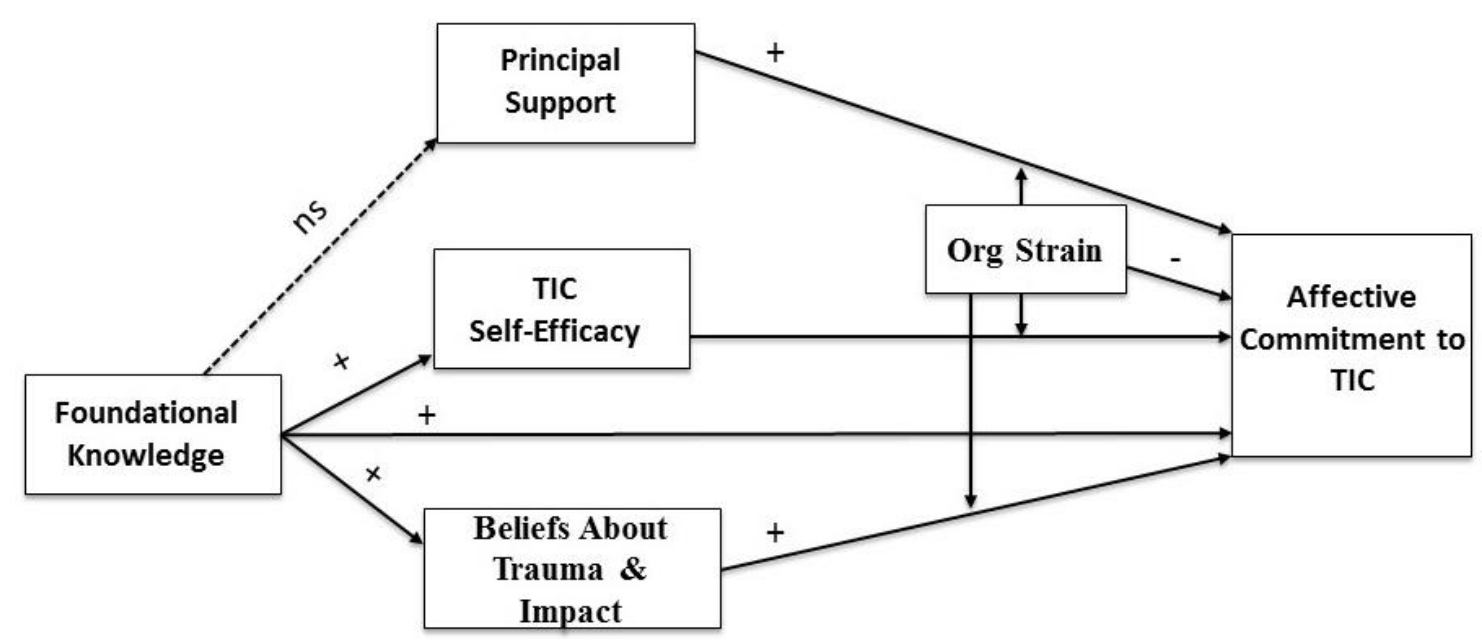

Figure 2. Full hypothesized model. Full model illustrates the proposed relationship between foundational knowledge about TIC, beliefs about trauma and its impact, beliefs about TIC self-efficacy, principal support, organizational strain, and affective commitment to TIC. Note: TIC $=$ trauma-informed care. OrgStrain $=$ organizational strain.

\section{Direct Effects}

Hypothesis 2. Foundational knowledge will have significant direct effects on affective commitment to TIC, TIC self-efficacy, and the belief about trauma and its impact.

However, there will not be a relationship between foundational knowledge and perceived principal support for TIC (see Figure 3). Specifically:

- 2a. Employees with greater foundational knowledge about trauma-informed care will have greater affective commitment to TIC.

- $2 b$. Employees with greater foundational knowledge about trauma-informed care will have greater TIC self-efficacy.

- 2c. Employees with greater foundational knowledge about trauma-informed care will have more TIC congruent beliefs about trauma.

- 2d. Foundational knowledge will not relate to an employee's perception of principal support for TIC. 


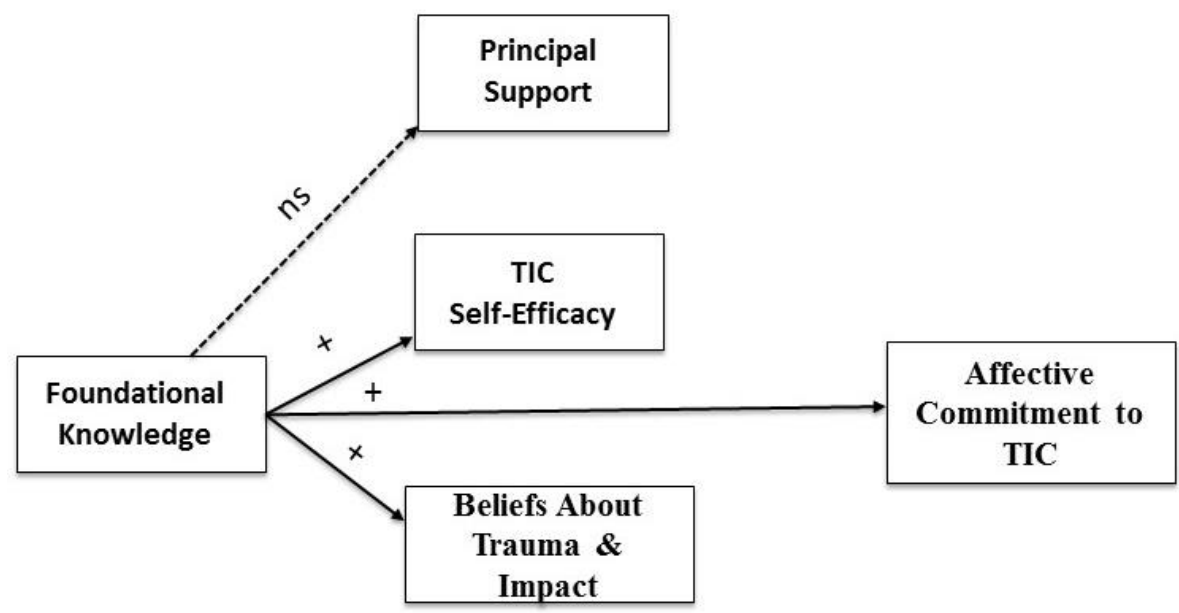

Figure 3. Direct Effects of Foundational Knowledge. Note: TIC = trauma-informed care . ns $=$ not significant.

Hypothesis 3. Beliefs about trauma, self-efficacy, perceived principal support, and organizational strain will significantly relate to the commitment to TIC (see Figure 4). Specifically:

- 3a. Employees with a greater belief that there is principal support for TIC will show greater affective commitment to TIC.

- $\quad 3 b$. Employees with greater TIC self-efficacy will show greater affective commitment to TIC.

- 3c. Employees with TIC congruent beliefs about trauma will show greater affective commitment to TIC.

- $3 d$. Employees who perceive greater organizational strain will be less committed to TIC. 


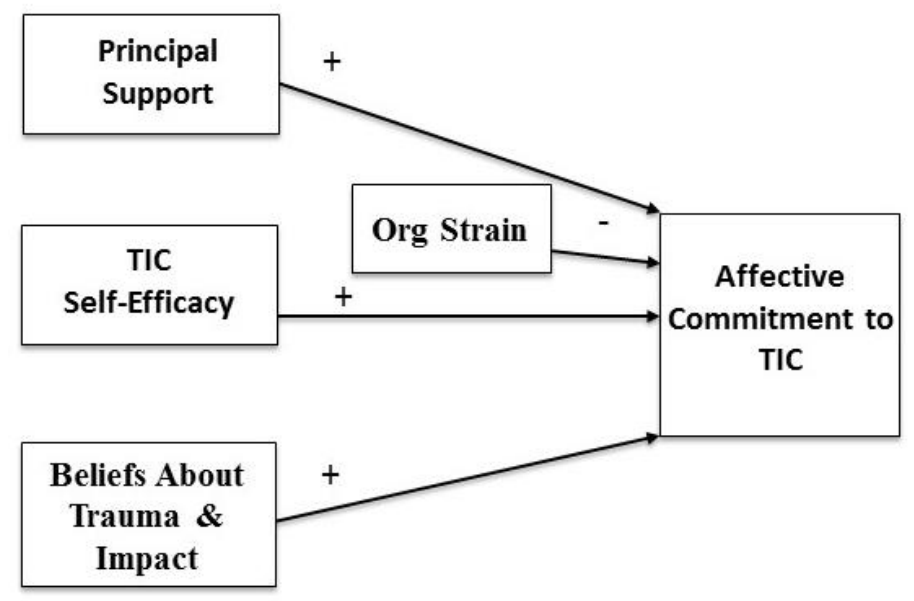

Figure 4. Direct effects of perceived principal support, TIC self-efficacy, beliefs, and organizational strain on affective commitment. Note: TIC $=$ trauma-informed care. OrgStrain = organizational strain.

\section{Indirect Effects}

Hypothesis 4. The relationship between foundational knowledge and affective commitment to TIC will be mediated by TIC self-efficacy and beliefs about trauma (see Figure 5). Specifically:

- 4a. Foundational knowledge will increase self-efficacy, which will lead to greater affective commitment to TIC.

- $\quad 4 b$. Foundational knowledge creates TIC congruent beliefs about trauma, which increases affective commitment to TIC. 


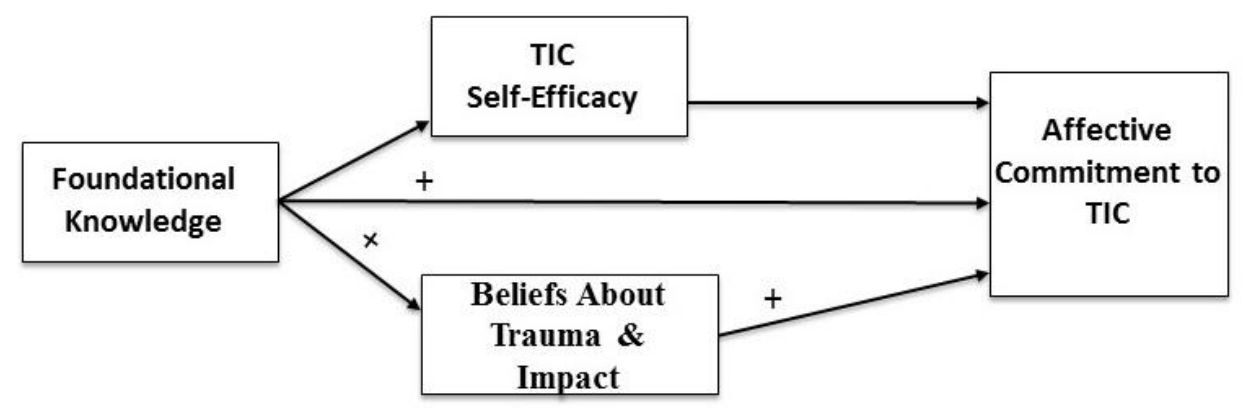

Figure 5. Mediated effects between knowledge and affective commitment through selfefficacy and beliefs about trauma. Note: TIC = trauma-informed care.

\section{Moderation}

Hypothesis 5. Organizational strain will moderate the relationship between affective commitment to TIC and perceived principal support, self-efficacy, and beliefs about trauma (see Figure 6). Specifically:

- 5a. Employees with a greater belief that there is principal support will show greater affective commitment to TIC when they perceive organizational strain to be low but not high.

- 5 b. Employees with a greater sense of self-efficacy will show greater affective commitment to TIC when they perceive organizational strain to be low but not high.

- 5c. Employees who have TIC congruent beliefs about trauma will show greater affective commitment to TIC when they perceive organizational strain to be low but not high. 


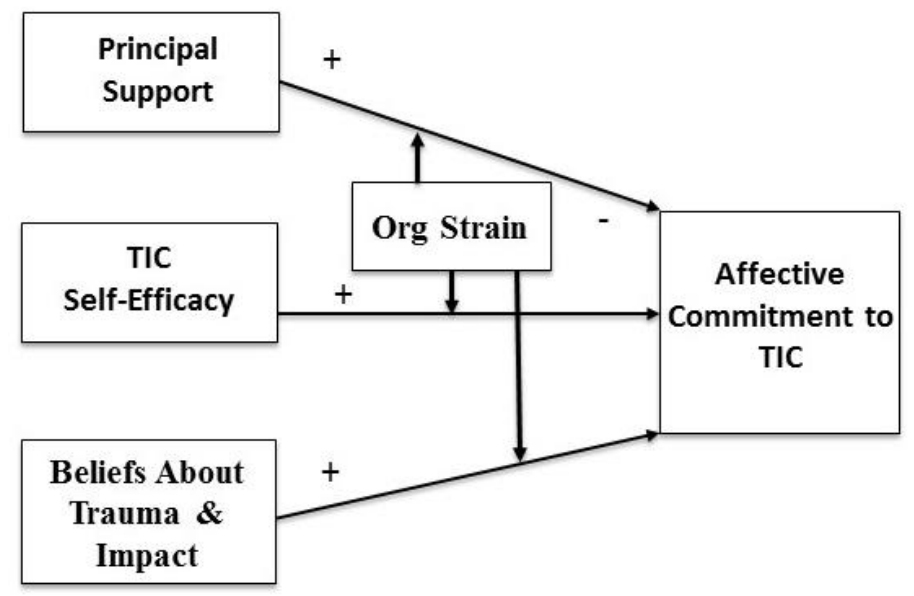

Figure 6. Moderated effects of organizational strain. Note: TIC $=$ trauma-informed care . OrgStrain $=$ organizational strain. 


\section{Chapter 4}

\section{Methods}

\section{Study Design}

This study used a cross-sectional survey design with quantitative data. A survey is an effective method for gathering the amount of data needed to address the study hypotheses using structural equation modeling. Quality results depend on both the sample and the survey items (Singleton, Straits, \& Straits, 2010). Questions were piloted for clarity and word choice. See the Instrumentation section for additional information.

In order to easily solicit voluntary and anonymous participation, an online survey was created using Qualtrics Software, version 2015 (Qualtrics, Provo, UT). Participants were solicited with an introductory email and a link to the online survey. The criteria for solicitation included attending a TIC training or working for an organization implementing this approach to service delivery. Training and education about TIC can be obtained through a variety of methods including webinars, print materials, and in-person trainings. In order to explore the relationship between knowledge and affective commitment to TIC, a variability in knowledge was desirable. However, a basic awareness was necessary to answer the questions. To this end, a filtering question was presented at the beginning of the survey asking about the respondent's awareness of TIC. Those who indicated that they were not aware of TIC were thanked for their participation and exited from the survey.

Those indicating awareness of TIC and a desire to participate in the survey were 
moved on to the informed consent form, which was included in the beginning of the survey as a question. The language from the informed consent was placed in the question text box with answer choices of yes or no. If respondents selected no, indicating that they did not consent to participate in the study, they were thanked and then exited from the survey. A selection of yes indicated that they were informed about the study and their rights as a participant and were willing to participate. Names and signatures were not solicited in order to ensure the respondent's anonymity.

After finishing the last question, the respondents were thanked for their participation and the survey ended. The completed, anonymous surveys are stored in Qualtrics so they could be downloaded for analysis. There was no interaction between the participant and the researcher. Nor was there any identifying information, with the exception of a computer-generated ID. The survey was open for 12 weeks as data were being collected. A reminder was sent after two weeks, regardless of whether the respondent had completed a survey. However, research suggests neither incentives nor reminders increase the response rate for organizational surveys (Baruch \& Holtom, 2008).

\section{Participants}

Sample recruitment. Study participants were recruited from several recent (2016) trainings or presentations on TIC offered by this researcher (in her role as a trainer for Trauma Informed Oregon) via email solicitation. The total number of individuals solicited was 420. First, participants were solicited from an all-staff training that had been attended by county employees who provide public health, mental health, and substance 
abuse services in many communities in Central Oregon. At the time of the survey, 252 individuals were working for the county health services organization and all of these employees were invited to participate. From this solicitation, 111 individuals completed the survey, a response rate of $44 \%$. Several weeks later, an email solicitation was sent to participants who had attended a TIC training earlier in the year, $\mathrm{n}=168$. These individuals represented public health home visiting services and early childhood. Twenty email addresses were no longer valid. From the 148 delivered email solicitations, 25 individuals completed the survey, a response rate of $17 \%$. Combined, the response rate was $34 \%$.

Sample characteristics. Seventy-three percent of participants $(n=86)$ self-reported as female, $10 \%(n=12)$ self-reported as male, and 17\% $(n=20)$ did not disclose gender. The participants ranged in age from 24 to 66 years old $(M=41)$. A majority (79\%) of the respondents represented the public or mental health fields. Slightly more than half $(n=60)$ worked in direct services while an additional one-third worked in management or administrative and support roles. Overall, the sample had a great deal of experience. Fewer than $4 \%$ had less than two years' experience in their field, and nearly $36 \%$ had more than 10 years of experience. Regarding prior training on trauma and traumainformed care, the respondents had been exposed to these topics through in-person training, webinars, and books. Across all three sources (in-person training, webinars, and books) respondents reported very little exposure on the topics of trauma and TIC $(\mathrm{Mdn}=2.3)$. Of those who had "some" or "a lot" of training, the most common source of information was through webinars $(n=67,63 \%)$ followed by books $(n=41,39 \%)$. Sixty- 
four percent of respondents indicated "none" or "very little" in-person training on these topics. A summary of demographic variables is provided in Appendix A.

\section{Instrumentation}

The Commitment to Trauma Informed Care survey contained questions measuring the constructs of interest, including affective commitment to TIC, foundational knowledge about TIC, beliefs about trauma and its impact, perceived self-efficacy regarding TIC, perceived principal support from peers and leaders, and organizational strain (stress and turbulence) in the workplace.

The survey items were grouped by construct in order to avoid confusion (Davis \& Venkatesh, 1996). This practice has been criticized for potential context error and inflated reliability alphas (Wilson \& Lankton, 2012); however, Goodhue and Loiacono (2002) note that these issues are not as concerning when measures are well-defined and supported in the research. Most constructs in this study were measured using valid and reliable scales. However, questions aimed at measuring Beliefs about Trauma and Foundational Knowledge about TIC were created specifically for this study. Therefore, these measures were piloted for clarity, word choice, and other considerations.

Clarity of questions. One of the areas of consideration was the use of verbs (e.g., know, understand, explain) and whether there was any difference in interpretation based on the word choice. The consensus was that any of these choices in wording was fine. Another consideration was answer choices. As a result of the piloting, it was decided that the questions should be posed in the form of a statement, such as I understand how 
trauma can affect relationships and attachment. The answer choices could then be in the form of agreement, consistent with the rest of the survey.

The beliefs about trauma scale risked being vague; therefore, making sure the questions were easy to understand was also a goal of piloting. Feedback was provided about clarity and word choices and these changes were incorporated into the survey.

Finally, for clarity and with permission from the authors of these instruments, the change referent ${ }^{3}$ was added directly into the question. A brief explanation was also included at the beginning of each section of the survey to provide clarification.

Likert scaling. According to the pilot test feedback, the six Likert answer choices were agreeable. Additionally, not sure was recommended as a response choice and was added to the survey. However, the order in which it was listed in Qualtrics put it outside the Likert range of strongly disagree to strongly agree, making its neutral quality less clear. From the preliminary data analysis, it appeared that individuals selected not sure when they neither agreed nor disagreed. Therefore, it was treated as a neutral center rather than as missing data.

\section{Measures.}

Affective commitment to trauma-informed care. Commitment is an important antecedent to behavioral change (Ajzen et al., 2009; Ajzen \& Fishbein, 2005).

\footnotetext{
${ }^{3}$ In the organizational change readiness literature, instrument items often keep the change unspecified, as is exemplified in this question from Armenakis et al. (2007), "I believe we can successfully implement this change." When a change is spelled out, this is referred to as the change referent.
} 
Herscovitch and Meyer (2002) created a measure of commitment to change that includes affective (six items), normative (six items), and continuance commitment (six items). Affective commitment translates to a "desire to support change based on beliefs about the benefits it brings" (Shin et al., 2012, p. 728). This subscale, which is related to championing behaviors, has an internal consistency reliability of $\alpha=.94$ and was included as the dependent or criterion variable in this study. With approval from the instrument creator, the reference to TIC was included in the question. "If the change can be clearly identified it is ok to specify it clearly in the instructions or the individual items" (J. Meyer, personal communication, August 8, 2016). Example questions included TraumaInformed Care is a good strategy for this organization. I think that management is [or would be] making a mistake by introducing Trauma-Informed Care. Answer choices were 1 =strongly disagree, $2=$ moderately disagree, $3=$ mildly disagree, $4=$ not sure, $5=$ mildly agree, $6=$ moderately agree, $7=$ strongly agree. The full list of questions is found in Appendix B.

Foundational knowledge for trauma-informed care. The knowledge measure captured information a respondent had received from a number of different sources, including formal face-to-face training, informal webinars and videos, print materials, and on the job mentoring or supervision. The Foundational Knowledge for Trauma-Informed Care Scale (see Appendix B) was created for the current study. This self-assessment instrument had an internal consistency reliability of $\alpha=.96$ and consisted of 30 questions measuring amount of knowledge on the topics of trauma in the workforce, principles and 
strategies of trauma-informed care, neurobiology of stress and trauma, the ACE study, historical oppression and intergenerational trauma, and issues of power and oppression related to the experience of trauma. Example questions included I understand the signs and symptoms of work-related stress including secondary traumatic stress, vicarious trauma, compassion fatigue, and burnout. I know the principles of Trauma-Informed Care. I understand that a stress response can be activated in the absence of real threat. The complete list of questions can be found in Appendix B. Answer choices were $1=$ completely untrue, $2=$ somewhat untrue, $3=$ somewhat true, $4=$ completely true. "True" was chosen instead of "strongly agree" because true is deemed a cognitive choice as opposed to agree, which conveys emotion. When a question requires that a respondent indicate how close his or her perception comes to the statement, a measure of truthfulness is often warranted (Fowler, 2013).

Principal support for trauma-informed care. The principal support variable in the study represented interest and buy-in from key individuals at an organization including leadership and peers as measured in the Organizational Change Recipients' Beliefs Scale (OCRBS; Armenakis et al., 2007). With an internal consistency reliability of $\alpha=.75$ (exploratory factor analysis), $\alpha=.69$ (confirmatory factor analysis), the authors suggested the marginal reliability coefficient might be a result of combining peers with leadership (Armenakis et al., 2007). For TIC, support is needed from both peers and leadership; therefore, each was measured in the current study.

Accordingly, six questions were used to measure principal support related to TIC, 
including the following examples: Most of my respected peers have embraced TraumaInformed Care. The top leaders support Trauma-Informed Care. Answer choices ranged from strongly disagree to strongly agree, as described earlier.

Change self-efficacy. Change self-efficacy was adopted from the Readiness for Organizational Change Scale (ROC; Holt, Armenakis, Feild, \& Harris, 2007). TIC is a transformational culture change, with unspecified changes in practice (that vary from person to person, place to place). ROC highlights the skills and confidence needed to feel efficacious and seemed more appropriate for this study than other efficacy scales (e.g., OCRBS) that are worded in reference to job performance and role. Cronbach's alpha for the ROC subscale efficacy is $\alpha=.82$ (Holt et al., 2007).

The change self-efficacy subscale in this study included the six items outlined in the ROC scale. In addition, one question was added that exactly mirrors the question "I have the skills that are needed to make this change work" but instead asked about the knowledge needed. Trauma-informed care requires both skill and knowledge; therefore, understanding an individual's confidence in both of these categories was important. This measure was hypothesized to mediate the relationship between foundational knowledge for TIC and affective commitment to TIC. Example questions include When we implement Trauma-Informed Care, I feel I can handle it with ease. When I set my mind to it, I can learn everything that will be required when Trauma-Informed Care is adopted. Answer choices ranged from strongly disagree to strongly agree, as described earlier. 
Beliefs about trauma and its impact. It was hypothesized that an important predictor in an individual's commitment to TIC was his or her beliefs about trauma, the impact of trauma on behaviors, and the role of the service system. These beliefs were hypothesized to directly and indirectly relate to commitment to TIC. To date, research exploring this concept has focused on attitudes about TIC itself, creating a gap in the literature. The Institute for Health and Recovery (2014) created an instrument that comes closest to tapping these beliefs. The questions created for this study were loosely adapted from the Staff Practice Survey instrument as part of the Developing Trauma-Informed Organizations: A Tool Kit (Institute for Health and Recovery, 2014). The Staff Practice Survey measures attitudes; however, these attitudes are limited to opinions about TIC delivery with clients and the potential for healing. Additionally, this tool is not empirically validated.

The Beliefs about Trauma and Its Impact Scale (see Appendix B) was created for the current study. This measure focused on relevant aspects of psychological trauma, including belief about prevalence, beliefs about trauma's impact on behavior and engagement, and belief that the service delivery system is involved in healing or perpetuating the trauma. All of these concepts are essential for TIC implementation. This self-assessment instrument consisted of 11 questions including the following examples: Many of the clients served by our agency have a history of trauma. Past trauma can influence current behavior of service recipients. Seeking and receiving services from our agency can be re-traumatizing for trauma survivors. The complete list of questions can 
be found in Appendix B. Answer choices ranged from strongly disagree to strongly agree, as described earlier.

It should be noted that the questions in the belief about trauma measure correspond to similar topics assessed in the knowledge scale. The difference is that the knowledge scale simply assesses the amount of knowledge an individual has received on a topic, compared to the belief scale that assesses his or her opinion (belief) about the topic. Further, the efficacy scale added an ability/capacity component that extended beyond the mere knowledge or opinion about the topic.

Organizational strain. This variable was composed of a four-item measure of organizational stress and a four-item measure of organizational turbulence, eight items total. Exploratory factor analysis was completed to ensure these two components load onto the same factor, permitting the variable to be treated as observed in the structural model.

Organizational stress was measured using the four stress items from the Organizational Climate subscale of the Organizational Readiness for Change instrument (ORC-D4; Lehman, Greener, \& Simpson, 2002). This subscale has been used extensively in research (Lehman, Greener, Rowan-Szal, \& Flynn, 2012; Weiner et al., 2008). The stress portion of the Organizational Climate subscale has an internal consistency reliability of $\alpha=.82$ (sample of directors, $\mathrm{n}=135$ ), $\alpha=.79$ (sample of staff, $\mathrm{n}=458$ ) and $\alpha=.90$ (with a program sample, $\mathrm{K}=111$ ). Example items included I am under too many pressures to do my job effectively. Staff members at my organization show signs of high 
stress and strain.

Organizational turbulence was defined as the "preponderance of changes going on in the organization at the same time as the focal change - changes that represent additional distractions and adaptation demands and thus form an important part of the context of individuals' reactions to a change" (Herold et al., 2007, p. 944). This concept was measured using the turbulence scale found in the study by Herold et al. (2007); internal consistency reliability is $\alpha=.76$. This measure had four items, including the following examples: This change toward Trauma-Informed Care is occurring during a turbulent time for our organization. This change toward Trauma-Informed Care would have been easier if we were not already dealing with a number of other changes. For both scales, the answer choices ranged from strongly disagree to strongly agree, as described earlier.

Demographic information. In addition to age and gender, demographic information was collected about years of experience, role, and amount of previous training. The full list is provided in Appendix B.

\section{Data Analysis}

Sample size and power analysis. Factor analysis and structural equation modeling (SEM) require large sample sizes (Kline, 2011). With an inadequate sample, especially in complex models with many parameters, the ability to appropriately reject the null hypothesis in the case of a true effect (e.g., power) is diminished. Kline (2011) noted that in addition to the probability value established for statistical significance, power relates to the reliability of the measures and estimation method. To ensure statistical power, Kline 
(2011) recommended a sample size close to 200. However, other researchers have recommended sample size be based on complexity of the model, suggesting 10-20 participants per observed variable. With factor analysis supporting the use of beliefs and organizational strain as observed variables (see factor analysis in the Results section), the hypothesized full model in this study included six observed variables, which, per these guidelines, would warrant a sample between 60 and 120. A sample size of 118 was adequate for the statistical analysis.

Data preparation. SEM is a statistical procedure primarily utilizing covariance between observed or latent variables in order to best explain the variance in a hypothesized model. Before testing a hypothesized model, a number of assumptions must be met. First, because this method is based on covariance, one of the most important considerations is collinearity between variables. Excessively high correlation between variables indicates redundancy and presents an opportunity to combine or eliminate variables (Kline, 2011). Due to possible overlap in measurement, knowledge, beliefs about trauma, and self-efficacy were tested for issues of collinearity. Second, the data must be free from extreme outliers, especially those that may be contributing to significant correlations. Missing data is a concern; however, there are several methods for handling this situation, described below. Finally, the assumption of univariate and multivariate normality needs to be considered for SEM. The method of estimation used by AMOS, maximum likelihood, assumes normality in the data. Data screening was done using SPSS 24. Findings are provided in Chapter 5. 
Missing data. Missing data can be problematic especially if the missing values are systematic rather than missing at random. There are several methods for handling randomly missing data. One method is to replace the missing data with a calculated score such as the mean or regression value (single imputation). This ad hoc method of dealing with missing data has disadvantages including a decrease in variance and correlations, both important measures in SEM (Byrne, 2016). Alternatively, estimating means and intercepts can be done in AMOS using a method called full information maximum likelihood (FIML). This theory-based approach uses an algorithm to estimate missing values and parameters. Although this method has been shown to outperform other methods (Byrne, 2016), there are some problems with replacing missing data in this way. First, the use of bootstrap resampling is not allowed when the missing data are replaced as part of the structural equation modeling. Second, modification indices (useful information when trying to improve model fit) are not available with missing data. For this study, given the advantages of bootstrap resampling, especially for mediated effects and non-normally distributed data (Kenny, 2016), mean imputation was the more desirable choice for replacing missing data.

Factor analysis. In order to explore whether beliefs and organizational strain could be treated as observed variables, exploratory factor analysis was conducted using SPSS 24. Composites were formed for these variables, and statistics were run using the sumscore. Findings are discussed in Chapter 5. 
Structural equation modeling (SEM). SEM is a method used to explore the relationships between multiple variables (both latent and observed) simultaneously. Because of its ability to account for error in the independent variable, it can be superior to multiple regression (Kline, 2011). Many techniques fall under the umbrella of SEM; however, they all rely on the following steps: 1) specification of the model, 2) identification of the model, 3) data collection and preparation, 4) estimation of the model, and 5) re-specification, if needed (Kline, 2011). Maximum likelihood, the default in AMOS, was the method of estimation. In addition, bootstrap resampling was performed with 1,000 samples drawn. Bias corrected confidence intervals were provided as an additional method for assessing fit. SEM was conducted using AMOS version 24 (Arbuckle, 1999).

Model specification. Specification is the first and most important step, as this is where the model is conceptualized (Kline, 2011). Depending on the number of variables, observations, and parameters, models will be simple or more complex. The current study was based on a structural path model, including both direct and indirect effects. The specified model had six observed variables, with $q=21$ observations. There were two exogenous variables (foundational knowledge and organizational strain), and four endogenous variables (principal support, self-efficacy, belief about trauma and its impact, and affective commitment to TIC). Among these variables, there were eight direct effects. Given this information, the number of parameters was 14 (direct effects plus covariance and variance). The degrees of freedom (the difference between the 
observations and number of parameters) was $d f_{\mathrm{M}}=21-14=7$.

Model identification. Identification of the model is the stage where it is determined that each parameter has a unique estimate and can be calculated by the computer program (Kline, 2011). Two requirements of identification include degrees of freedom greater or equal to zero and scale assignments for each latent variable.

The degrees of freedom of the specified model equaled 7; therefore, this criterion was met. Further, because this model was recursive ${ }^{4}$, it was automatically identified (Kline, 2011). Recursive models have uncorrelated disturbances and one-directional causation.

Model estimation. Once the data are ready for analysis, the computer compares the data to the model, estimating the fit. Maximum likelihood (ML) is the default technique for SEM and can be used if the following assumptions are met: scores are independent, endogenous variables have normal distribution, exogenous variables are independent, and specification of the model is correct (Kline, 2011). If the data are a good fit to the model, then parameters can be explained. Model testing reflects an overall model statistic (with chi-square distribution) as well as the approximate fit indices, including absolute, incremental, parsimony-adjusted, and predictive. Among the most common approximate fit indices, and the ones reported in this study, are the root-mean square error of approximation (RMSEA; Steiger, 1990) and the Comparative Fit Index (CFI; Bentler,

\footnotetext{
4 The hypothesized model has uncorrelated disturbances; however, the re-specified model adds covariation between error terms. The re-specified model is considered a bow-free pattern and is still recursive because the correlated error terms relate to endogenous variables without direct effects (Kline, 2011).
} 
1990). Both are appropriate indices when using ML (Herscovitch \& Meyer, 2002), but each has unique measurement approaches and assumptions. RMSEA, in confirming "badness of fit," estimates the discrepancy between the data and the model. Good fit is indicated by a value less than .05. Poor fit is indicated with a value greater than .10 (Browne \& Cudeck, 1993). This index is parsimony-adjusted; therefore, as degrees of freedom increase, the RMSEA value will decrease. CFI is an incremental fit index, comparing the model to a baseline (i.e. the incremental change). Values for CFI close to 1.0 indicate a good fit. If the data are not a good fit, then re-specification is needed. 


\section{Chapter 5}

\section{Results}

This chapter presents the results from the analysis described in Chapter 4. A number of statistical analyses were run to explore and describe the sample, address statistical assumptions, and address the hypotheses outlined in Chapter 3. Analysis was conducted using IBM's SPSS 24 and AMOS 24. The first portion of this chapter describes the sample and addresses assumptions underlying the statistical methods used in this study, including factor analysis and SEM. The second portion of the chapter addresses the hypotheses directly.

\section{Preliminary Data Analysis}

Missing data. Most of the variables showed low amounts of missing data, with the exception of knowledge and turbulence (see Table C1). Eight percent (8\%) of the knowledge data were missing and 7\% of the turbulence data were missing. Kline (2011) suggested that if missing data are low (e.g., $5 \%$ ), there will not be a noticeable difference between the methods of handling the absence. However, the model was also fit using FIML (see Tables 3 and 4), which produced similar results.

In addition to the sporadic data missing at random, a number of cases had such extensive missing data that they were eliminated listwise (i.e., the case is eliminated from the data set). The first question asked whether a participant was familiar with traumainformed care. If the respondent answered "no" to this question, he or she was taken to the end of the survey and thanked for participating. This occurred in 13 cases, which 
were subsequently eliminated from analysis. Further, five additional cases were eliminated because the respondents only completed the questions measuring affective commitment to TIC.

\section{Descriptive statistics of hypothesized model.}

Means. The survey resulted in ordinal (Likert scale) data; however, because the number of answer choices was greater than four, it was acceptable to treat it as continuous data (Byrne, 2016). The following is a brief overview of the average responses for individual variables.

Affective commitment to TIC was strong, with nearly $47 \%$ respondents selecting strongly agree for each question of the measure. On average, respondents moderately to strongly agreed that they were committed to TIC and thought it was a good idea for their organization $(\mathrm{M}=6.28)$. Only $9 \%$ were not sure or disagreed that they were committed to TIC. Overall, beliefs were congruent with TIC $(M=5.91)$. However, respondents only mildly agreed that service settings and programs could be re-traumatizing for service recipients and staff $(M=5.11)$. On the TIC self-efficacy measure, respondents mildly to moderately agreed that they had the skills, knowledge, and ability needed to implement TIC (M=5.46). Further, within their organizations they agreed mildly that their peers and leadership encouraged and modeled TIC (M=5.13). Respondents mildly to moderately agreed that high workload, pressure, and stress were present at their organization $(M=5.29)$. Further, organizational turbulence (change) did not seem to be an impediment 
to TIC, $(M=4.84)$. Means, standard deviations, and the range of answers are summarized in Appendix C in Table C1.

Perceived knowledge, whether through webinars, books, or in-person training, was strong. Across all topics, respondents indicated that it was somewhat to completely true that they understood and could explain the content related to trauma and TIC $(\mathrm{M}=3.22)^{5}$. The topic area with which respondents had the most perceived knowledge was historical and systemic oppression, followed by the neurobiology of trauma. The topic area with which they had the least perceived knowledge was the principles of TIC (M=2.89).

Table 1.

Mean Values for Specific Knowledge Topics

\begin{tabular}{lcc}
\hline \multicolumn{1}{c}{ Topic } & Mean & $\begin{array}{c}\text { Standard } \\
\text { Deviation }\end{array}$ \\
\hline Total Knowledge & 3.22 & .51 \\
Work-related stress & 3.12 & .52 \\
Principles of TIC & 2.89 & .63 \\
Neurobiology of trauma & 3.41 & .53 \\
ACE study and findings & 3.05 & .84 \\
Intergenerational effects & 3.04 & .80 \\
Historical and systemic & 3.47 & .64 \\
oppression & & \\
\hline
\end{tabular}

Note: TIC $=$ trauma-informed care. ACE $=$ adverse childhood experiences.

\footnotetext{
${ }^{5}$ Knowledge was measured using a 4-point Likert scale (completely true, somewhat true, somewhat untrue, and completely untrue). All other variables were measured using a 7-point Likert scale (strongly agree, moderately agree, mildly agree, not sure, mildly disagree, moderately disagree, and strongly disagree).
} 
Normality and outliers. Multivariate normality and the absence of outliers are important considerations for SEM. A visual inspection of the data using histograms and p-p plots to check for normality indicated some areas of concern. Most of the variables were negatively skewed indicating a predominance of responses at the higher end of the scale. Positive kurtosis was also a concern, as many of the variables had a leptokurtic distribution. This suggested that answer selections were mostly at the extreme ends of the scale (either agreeing strongly or disagreeing strongly). This was especially the case for the dependent variable, affective commitment to TIC. Forty-seven percent of respondents $(n=55)$ strongly agreed with every question on the affective commitment measure.

In addition to a visual inspection, skew and kurtosis were checked quantitatively using a $\mathrm{Z}$ score distribution. The $\mathrm{Z}$ scores for skew indicated that all variables, except turbulence, had significant negative skew. In addition, many of the variables had significant kurtosis, which is a greater concern considering SEM is based on covariance (see Appendix C, Table C2). Principal support and organizational strain had significant kurtosis at $p<.05$ level. Affective commitment, beliefs, and TIC self-efficacy (SelfEff) had significant kurtosis, $p<.001$. Fortunately, knowledge, beliefs about services, stress, and turbulence were not significantly kurtotic.

The $\mathrm{Z}$ statistic, described above, is a measure of univariate skew and kurtosis. Of more importance is the multivariate nature of the data, which is information provided on AMOS output. The critical ratio for multivariate skew and kurtosis should be no greater 
than 5.0 for normally distributed data. The critical ratio for kurtosis of these data was 12.66 , indicating nonnormality.

Given nonnormality, there are several approaches for handling this situation. One reliable method is to use a scaling correction, such as Satorra-Bentler chi-square (Satorra $\&$ Bentler, 1988) in order to adjust parameter estimates and $\chi^{2}$. Unfortunately, this method is not available in AMOS. Alternatively, the use of bootstrap resampling can offer a strategy for nonnormality within the data and can be more reliable than ML if the distribution is nonnormal (Kline, 2011). Bootstrapping is advantageous because it "allows the researcher to assess the stability of parameter estimates and thereby report their values with a greater degree of accuracy” (Byrne, 2016, p. 369).

Collinearity is another consideration when using SEM and can be checked by reviewing the correlations. As seen in Table 2, most of the variables showed strong correlation but not to a level of worry. Of particular concern were the correlations between knowledge, beliefs, and self-efficacy because of the possible overlap in measurement. While these correlations were significant, they were not indicating redundancy in the measure. Unsurprisingly, organizational strain was highly correlated with its two subscales but for the SEM analysis, only the composite (OrgStrain) was used. Tolerance and variance inflation factor (VIF) are indicators of problematic collinearity (Pallant, 2010). Tolerance values less than .10 indicate a concern, and VIF values above 10 indicate a concern. None of the variables met these two thresholds (see Appendix C, Table C3). 
Table 2.

Pearson Correlations for Variables in the Model

\begin{tabular}{|c|c|c|c|c|c|c|c|c|}
\hline & $\begin{array}{l}\text { Affective } \\
\text { Commitment }\end{array}$ & Knowledge & Beliefs & $\begin{array}{l}\text { Self } \\
\text { Efficacy }\end{array}$ & $\begin{array}{l}\text { Principal } \\
\text { Support }\end{array}$ & $\begin{array}{l}\text { Organizational } \\
\text { Strain }\end{array}$ & Stress & Turbulence \\
\hline $\begin{array}{l}\text { Affective } \\
\text { Commitment }\end{array}$ & 1.00 & $.602 * *$ & $.576 * *$ & $.651 * *$ & $.597 * *$ & .008 & $.258 * *$ & $-.278 * *$ \\
\hline Knowledge & & 1.00 & $.385 * *$ & $.564 * *$ & $.279 * *$ & .103 & $.246 *$ & -.065 \\
\hline Beliefs $^{\mathrm{a}}$ & & & 1.00 & $.497 * *$ & .171 & $.246^{*}$ & $.480 * *$ & -.102 \\
\hline Self-Efficacy & & & & 1.00 & $.459 * *$ & -.089 & .134 & $-.277 * *$ \\
\hline $\begin{array}{l}\text { Principal } \\
\text { Support }\end{array}$ & & & & & 1.00 & $-.221 *$ & -.083 & $-.271 * *$ \\
\hline $\begin{array}{l}\text { Organizational } \\
\text { Strain }\end{array}$ & & & & & & 1.00 & $.810 * *$ & $.783 * *$ \\
\hline Stress & & & & & & & 1.00 & $.269 * *$ \\
\hline Turbulence & & & & & & & & 1.00 \\
\hline
\end{tabular}

${ }^{a}$ Beliefs is the observed variable resulting from factor analysis. This variable contains 10 of the items rather than 11 (see factor analysis section).

$* p<.05, * * p<.01$ 
Finally, the presence of outliers can be a problem. According to the Mahalanobis distance (D) statistic, there were three cases that exceeded the 20.52 threshold for five independent variables. The Cook's distance gives an indication whether these values are heavily influencing the results and values greater than 1.0 can be problematic (Pallant, 2010). The maximum value for Cook's Distance was .238, well below the threshold.

\section{Developing Composite Variables}

In order to investigate whether beliefs and organizational strain could be used as observed variables in this analysis, exploratory factor analysis was conducted using principal components analysis (PCA).

Beliefs as an observed variable in the model. Eleven questions measuring three aspects of beliefs about trauma and its impact were included in the survey (see Appendix D, Figure D1). The three areas included beliefs about the prevalence of trauma, beliefs about its impact (e.g., effect on behavior), and beliefs that service systems can be retraumatizing for trauma survivors.

Given the presence of many strong correlations between these items (greater than .30, see Appendix D, Table D1), factor analysis was appropriate for these data (Pallant, 2010). Further, two other measures supported a factor analysis of these items. Bartlett's Test of Sphericity was significant, $\chi^{2}(55)=442.75, p=.000$, and the Kaiser-Meyer-Olkin (KMO) measure of sampling adequacy was .794, which is above the recommended .6 minimum value. Missing values were deleted pairwise. 
Principal component analysis with oblique (oblimin) rotation was used to conduct the exploratory factor analysis. Results are shown in Appendix D, Table D2. Initial analysis, based on eigenvalues greater than 1 and a review of the screeplot, suggested two components for these factors, with one item loading onto a third component (and explaining an additional $9 \%$ of the variance overall). The proposed components seemed to reflect beliefs about the prevalence and impact of trauma and beliefs that service settings can be re-traumatizing. With these two components, $54 \%$ of the variance was explained. Ideally, components have at least three items with factor loadings exceeding .512 (Field, 2009; Pallant, 2010).

Despite the numeric support of two components, theoretically trauma-informed care rests on the understanding of the prevalence and impact of trauma as well as the role that service settings and programs play. Furthermore, the proposed model in this study relied on one measure of beliefs. For this reason, another analysis was conducted with the factors fixed to one. The resulting solution explained $37 \%$ of the variance. The results are presented in Table 3. 
Table 3.

Component Matrix for Beliefs Scale - Fixed Factor Principal Components Analysis

\begin{tabular}{|c|c|}
\hline & $\begin{array}{c}\text { Component } \\
1\end{array}$ \\
\hline $\begin{array}{l}\text { S5: Working with trauma survivors can result in } \\
\text { work-related stress such as vicarious trauma. }\end{array}$ & .786 \\
\hline $\begin{array}{l}\text { P1: Many of the clients served by our agency have } \\
\text { experienced psychological trauma. }\end{array}$ & .757 \\
\hline $\begin{array}{l}\text { P2: Many of the staff in our agency have } \\
\text { experienced psychological trauma. }\end{array}$ & .745 \\
\hline $\begin{array}{l}\text { I4: When staff have experienced psychological } \\
\text { trauma (current or in the past), this can influence } \\
\text { their current behavior at work. }\end{array}$ & .703 \\
\hline $\begin{array}{l}\text { I1: Many problematic behaviors (such as substance } \\
\text { abuse) start as a way to cope with emotionally } \\
\text { traumatizing experiences. }\end{array}$ & .671 \\
\hline $\begin{array}{l}\text { S1: Seeking and receiving services from our } \\
\text { agency can be re-traumatizing for trauma } \\
\text { survivors. }\end{array}$ & .596 \\
\hline $\begin{array}{l}\text { S2: Our service setting does not create } \\
\text { psychological trauma for our service recipients. }\end{array}$ & .502 \\
\hline $\begin{array}{l}\text { I2: Past experiences of psychological trauma (for } \\
\text { instance, in childhood) cannot be linked to current } \\
\text { problematic behaviors in adulthood. }\end{array}$ & .499 \\
\hline $\begin{array}{l}\text { I3: When service recipients have experienced } \\
\text { psychological trauma (current or in the past), this } \\
\text { can influence their current behavior. }\end{array}$ & .451 \\
\hline $\begin{array}{l}\text { S3: Our programs and services do not create } \\
\text { psychological trauma for our service recipients. }\end{array}$ & .449 \\
\hline
\end{tabular}

Note: This represents the revised principal components analysis with oblimin rotation and factor fixed to one. The items are listed by letter, $\mathrm{P}=$ prevalence questions, $\mathrm{S}=$ service system questions, and $\mathrm{I}=\mathrm{impact}$ of trauma questions. Service question 4 "Service providers can absorb the psychological trauma of service recipients" was removed because the score of .253 was below the .40 cutoff. 
Given a one-factor solution, all of the items adequately loaded onto the component, with the exception of S4: Service providers can absorb the psychological trauma of service recipients. This item was removed from the measure. The remaining 10 items combined to form the observed variable beliefs used in the path analysis. The 10 items comprising beliefs explained $40 \%$ of the variance. Additionally, beliefs had high reliability, Cronbach's $\alpha=.81$.

\section{Organizational Strain (OrgStrain) as an observed variable in the model. The} proposed composite variable, organizational strain, was composed of two four-question instruments (see Appendix D, Figure D2). One instrument measured workplace stress and the other measured organizational turbulence (change).

The correlation matrix (see Appendix D, Table D3) showed strong correlations between items on each of the scales; however, the correlations between the two scale items was weak. Two other measures indicating whether these items were appropriate for factor analysis suggested this approach was suitable for these data. Bartlett's test of sphericity was significant, $\chi^{2}(28)=335.28, p=.000$, and the Kaiser-Meyer-Olkin (KMO) measure of sampling adequacy was .737, above the .6 minimum value (Pallant, 2010).

For this exploratory factor analysis, principal component analysis was conducted using orthogonal rotation (varimax) because of the low correlation between measures. Results are shown in Appendix D, Table D4. A review of the screeplot and eigenvalues 
suggested two separate components. One component explained $41 \%$ of the variance and the second component explained $23 \%$.

In the current study, the hypothesis was that organization strain (composed of stress and turbulence) would moderate the relationships between affective commitment to TIC and knowledge, beliefs, self-efficacy, and perceived principal support (hypothesis 5). Therefore, in accordance with the need to have one measure of strain and the theoretical support that stress and turbulence are related constructs (Herold et al., 2007), a fixed factor analysis was conducted (see Table 4). 
Table 4.

Component Matrix for Organizational Strain - Fixed Factor Principal Components Analysis

\begin{tabular}{|c|c|}
\hline & $\begin{array}{c}\text { Component } \\
1\end{array}$ \\
\hline $\begin{array}{l}\text { Stress 3: Staff members at my agency show signs } \\
\text { of high stress and strain. }\end{array}$ & .752 \\
\hline $\begin{array}{l}\text { Stress 4: Staff frustration is common where I } \\
\text { work. }\end{array}$ & .733 \\
\hline $\begin{array}{l}\text { Stress 2: I am under too many pressures to do my } \\
\text { job effectively. }\end{array}$ & .676 \\
\hline $\begin{array}{l}\text { Turbulence 4: This change toward Trauma- } \\
\text { Informed Care would have been easier if we were } \\
\text { not already dealing with a number of changes. }\end{array}$ & .628 \\
\hline $\begin{array}{l}\text { Turbulence } 3 \text { : We were still trying to digest earlier } \\
\text { changes when we embarked on Trauma-Informed } \\
\text { Care. }\end{array}$ & .616 \\
\hline $\begin{array}{l}\text { Turbulence 1: This change toward Trauma- } \\
\text { Informed Care is occurring during a turbulent time } \\
\text { for our organization. }\end{array}$ & .616 \\
\hline $\begin{array}{l}\text { Stress1: The heavy staff workload reduces the } \\
\text { effectiveness of my program. }\end{array}$ & .532 \\
\hline $\begin{array}{l}\text { Turbulence 2: This change toward Trauma- } \\
\text { Informed Care suffers from too many other } \\
\text { distractions. }\end{array}$ & .497 \\
\hline
\end{tabular}

With one component, these items still had strong factor loadings and explained $41 \%$ of the variance. Furthermore, the reliability of these items was high, Cronbach's $\alpha=.78$. 


\section{Hypothesis Testing}

The hypotheses outlined in Chapter 3 were addressed using a number of statistical methods including structural equation modelling, analysis of variance, and independent sample $t$-tests. The results are presented below.

\section{Hypothesis 1: The hypothesized full structural path model (including all direct,} indirect, and moderated effects) will be a good fit for the data and will explain a significant amount of variance in affective commitment to TIC. Figure 7 illustrates the full hypothesized model analyzed in this study. Based on the results of the factor analysis for beliefs and organizational strain, these two variables were included as observed variables.

According to the model fit summary and indices, the hypothesized (original) full model was not a good fit. Specifically, the hypothesized covariance matrix was significantly different from that of the population, $\chi^{2}(7)=52.709, p=.000$. Based on the modification indices (MI) and a nonsignificant estimate, $b=.009$, $\mathrm{SE}=.054, p=.862$, model improvement resulted from removing organizational strain (OrgStrain).

Additionally, MI suggested covariance be added between Err1 and Err2 and between Err 2 and Err3. Covariance between error terms indicates that these endogenous variables "share at least one common omitted cause" (Kline, 2011, p.107). According to Gaskin (2016), error terms are allowed to covary in a structural model if they are not causally correlated but rather are systematically correlated. In other words, the correlation results 
from respondents answering the scale items in a similar way. Systematic correlation rather than causal correlation is likely the case in these data. Therefore, the error terms were allowed to covary.

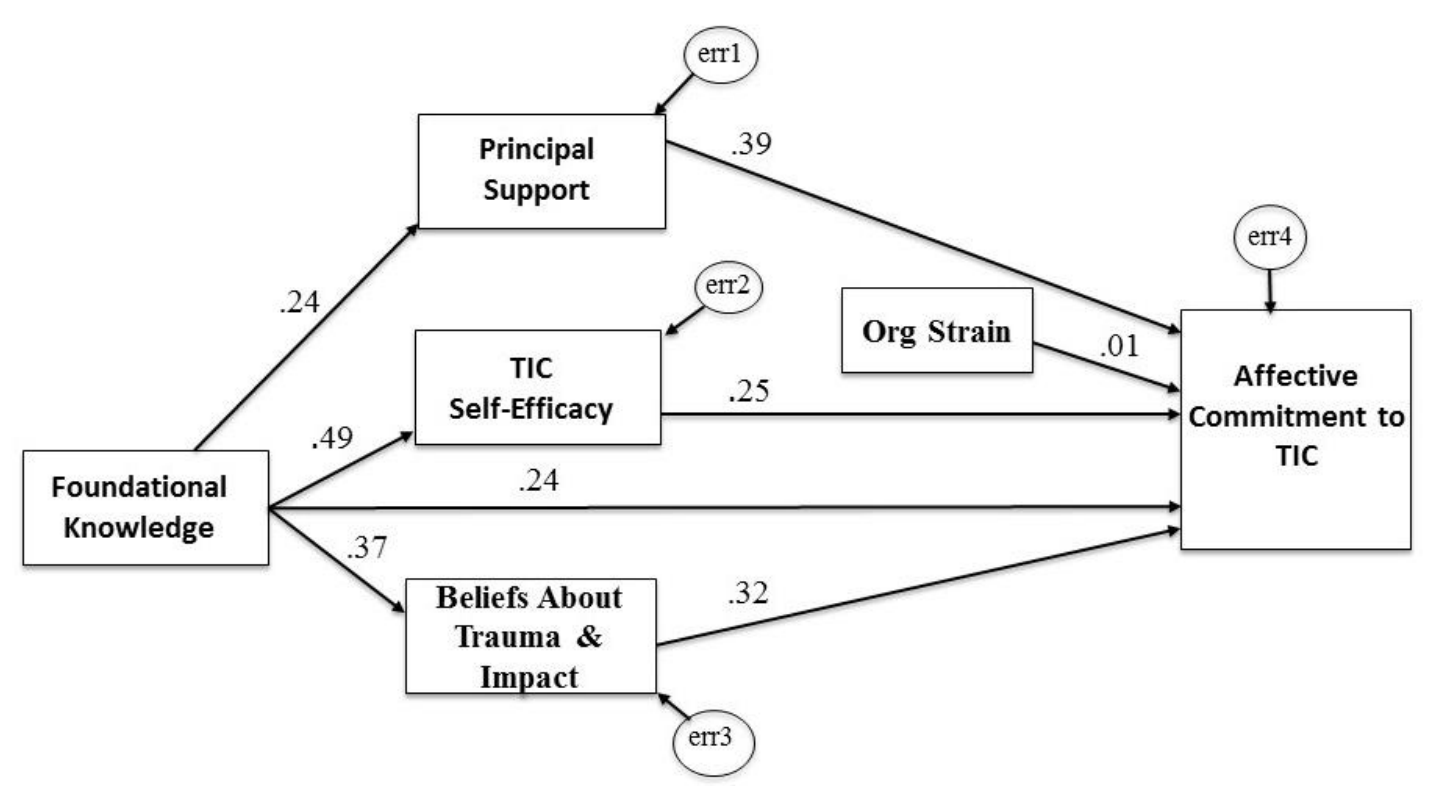

Figure 7. Standard estimates of the hypothesized model. Note: TIC $=$ trauma-informed care. OrgStrain $=$ organizational strain.

These changes resulted in a re-specified model that was not significantly different from the population model, $\chi^{2}(1)=.672, p=.412$ (see Figure 8 ). In addition to a nonsignificant chi-square value, the indices indicated a good fit (Kenny, 2015). The comparative fit index (CFI) was 1.00, which is greater than the recommended value of .96. The root mean square error of approximation (RMSEA) also supported an excellent fit with a value of .000. PCLOSE was not significant ( $\mathrm{p}=.477)$, which is desirable; however, the confidence interval was wide (e.g., straddling .05), indicating a less precise RMSEA value. This can result from having too few degrees of freedom in the model. 


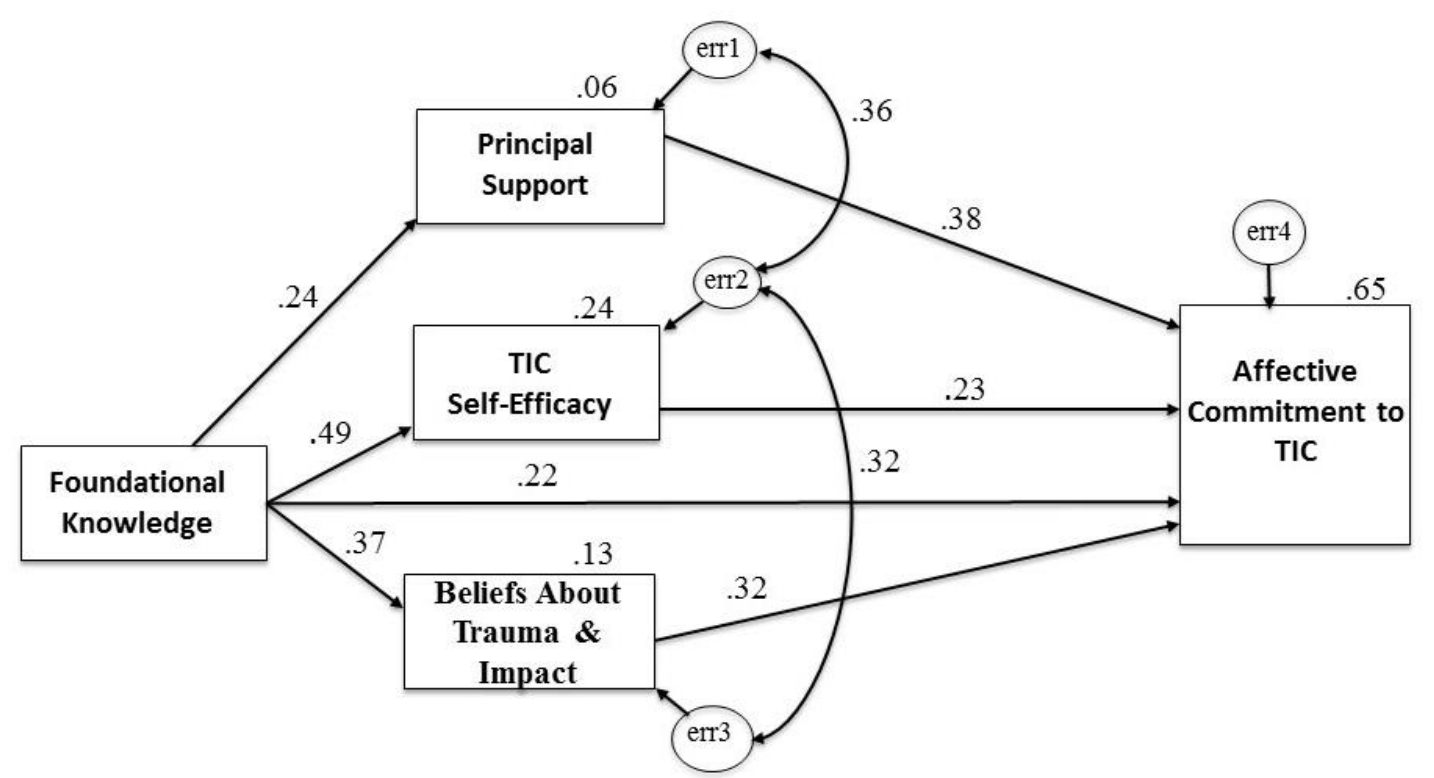

Figure 8. Standard estimates of re-specified model. Note: TIC $=$ trauma-informed care.

Parameter estimates were appropriate. CRs were all above 2.695. Standard errors were all small, which is good. No additional problems were highlighted by the modification indices.

Using ML, AMOS performed a bootstrap with 1,000 samples. In completing the 1,000 samples, 19 iterations were needed. All of the iterations were done using method 1 , which is a fast and reliable algorithm. Most of the samples were complete with 7-11 iterations.

For comparison, a model was tested using the FIML approach to missing data (see Table 5). 
Table 5.

Model Fit Indices

\begin{tabular}{lrrrrr}
\hline & $\chi^{2}$ & df & $p$ & CFI & RMSEA \\
\hline Hypothesized model $^{\mathrm{a}}$ & 52.709 & 7 & .000 & .790 & .236 \\
Final model $^{\mathrm{b}}$ & .672 & 1 & .412 & 1.000 & .000 \\
Comparison model $^{\mathrm{c}}$ & .503 & 1 & .478 & 1.000 & .000 \\
\hline
\end{tabular}

Note: $\chi^{2}=$ chi square. $\mathrm{df}=$ degrees of freedom. $P=$ probability value. $\mathrm{CFI}=$ comparative fit index. RMSEA $=$ root mean square error of approximation.

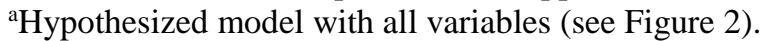

${ }^{b}$ Respecified model (see Figure 8) fit using mean imputed missing values and bootstrap resampling. ${ }^{\mathrm{c} C}$ Comparison model to the final model fit with FIML method for missing values.

With this approach, AMOS uses an algorithm to extrapolate missing values, but unfortunately, bootstrap resampling is unavailable. It's important to ensure that the model fit and indices are similar despite different approaches for missing data. The standardized estimates were quite similar between the model with mean imputation and the model using FIML (see Table 6). The method of mean imputation appeared to be slightly more conservative in its estimates than the FIML approach. The model using mean imputation was retained as the bootstrap resampling technique adds confidence in cases of nonnormal distribution (Byrne, 2016). 
Table 6.

Comparison of Estimates Using Full Information Maximum Likelihood and Mean Imputation

\begin{tabular}{|c|c|c|}
\hline \multirow[b]{2}{*}{ Direct effects } & $\beta$ & $\beta$ \\
\hline & FIML & MeanImp \\
\hline Knowledge $\rightarrow$ Affective $\left(c^{\prime}\right)$ & .286 & .223 \\
\hline Knowledge $\rightarrow$ Beliefs $\left(a_{1}\right)$ & .362 & .367 \\
\hline Knowledge $\rightarrow$ SelfEff $\left(a_{2}\right)$ & .575 & .493 \\
\hline Knowledge $\rightarrow$ PrinSupp $\left(a_{3}\right)$ & .320 & .242 \\
\hline Beliefs $\rightarrow$ Affective $\left(b_{1}\right)$ & .337 & .316 \\
\hline SelfEff $\rightarrow$ Affective $\left(b_{2}\right)$ & .132 & .234 \\
\hline PrinSupp $\rightarrow$ Affective $\left(b_{3}\right)$ & .405 & .375 \\
\hline \multicolumn{3}{|l|}{ Indirect effects } \\
\hline Knowledge $\rightarrow$ Affective $\left(a_{1} x b_{1}\right)$ & .122 & .116 \\
\hline Knowledge $\rightarrow$ Affective $\left(a_{2} x b_{2}\right)$ & .076 & .115 \\
\hline Knowledge $\rightarrow$ Affective $\left(a_{3} x b_{3}\right)$ & .130 & .091 \\
\hline Knowledge $\rightarrow$ Affective full model & .328 & .322 \\
\hline \multicolumn{3}{|l|}{ Total effect } \\
\hline Knowledge $\rightarrow$ Affective $\left(c_{1}\right)$ & .408 & .339 \\
\hline Knowledge $\rightarrow$ Affective $\left(c_{2}\right)$ & .362 & .338 \\
\hline Knowledge $\rightarrow$ Affective $\left(c_{3}\right)$ & .416 & .314 \\
\hline Knowledge $\rightarrow$ Affective full model & .613 & .545 \\
\hline \multicolumn{3}{|l|}{ Covariance } \\
\hline Err1 $\leftrightarrow$ Err2 & .351 & .365 \\
\hline Err2 $\leftrightarrow$ Err3 & .352 & .324 \\
\hline
\end{tabular}

Note: $\beta=$ standardized estimates, FIML $=$ full information maximum likelihood, MeanImp $=$ mean imputation. PrinSupp = principal support. SelfEff $=$ self-efficacy.

Overall, this model resulted in many significant effects. In fact, the amount of variance in affective commitment to TIC explained by the full model was $65 \%$. The mediated relationships between knowledge and affective commitment through TIC self- 
efficacy, beliefs about TIC, and perceived principal support explained $30 \%$ of the variance in affective commitment. All direct effects were significant in a positive direction meaning an increase in one variable caused an increase in the second variable. A discussion of the direct, indirect, and moderating effects follows.

\section{Hypothesis 2: Knowledge will have direct effects on the variables in the model.}

Specifically, knowledge will have a significant (positive) direct effect on affective commitment, TIC self-efficacy, and beliefs; however, it will not have a significant direct effect on principal support.

As hypothesized, knowledge significantly predicted TIC self-efficacy, beliefs about trauma, and affective commitment to TIC. With the greatest effect on TIC self-efficacy ( $\beta$ $=.493, p<.01)$, knowledge accounted for $24 \%$ of self-efficacy's variance. Knowledge also had a strong effect on beliefs $(\beta=.367, p<.01)$, accounting for $13 \%$ of the variance in this variable. Although a significant effect, knowledge only uniquely explained $5 \%$ of the variance in affective commitment $(\beta=.223, p<.01)$. However, as discussed below, knowledge had a greater impact through the mediated effects.

One of the surprising results, and contrary to the hypothesis, was the significant effect knowledge had on principal support $(\beta=.223, p<.01)$. Although this relationship only predicted $6 \%$ of the variance in principal support, it was an unexpected outcome. As a result, the mediated relationship between knowledge and affective commitment through principal support was considered as part of the model. 


\section{Hypothesis 3: The mediating and moderating variables in the model will have} direct effects on affective commitment to TIC. Beliefs about trauma, TIC self-efficacy, and perception of principal support of TIC from peers and leadership significantly predicted affective commitment. Of the three, principal support showed the strongest relationship such that a one-unit increase in perceived support corresponded to a nearly half unit $(b=.413)$ increase in affective commitment, and it accounted for $14 \%$ of the variance in this variable. Self-efficacy had the weakest relationship (although still statistically significant, $\beta=.234, \mathrm{p}<.01$ ), only increasing affective commitment by .235 for a one-unit increase in self-efficacy.

\section{Hypothesis 4: TIC self-efficacy and beliefs about trauma will mediate the} relationship between foundational knowledge and affective commitment to TIC. Hypothesis 2 and 3 verified significant direct effects in the model, a prerequisite to proposing mediated effects (MacKinnon, 2008). Because the direct effect between foundational knowledge and principal support was significant, an indirect pathway from foundational knowledge to affective commitment through principal support was added to the model (see Figure 9). 


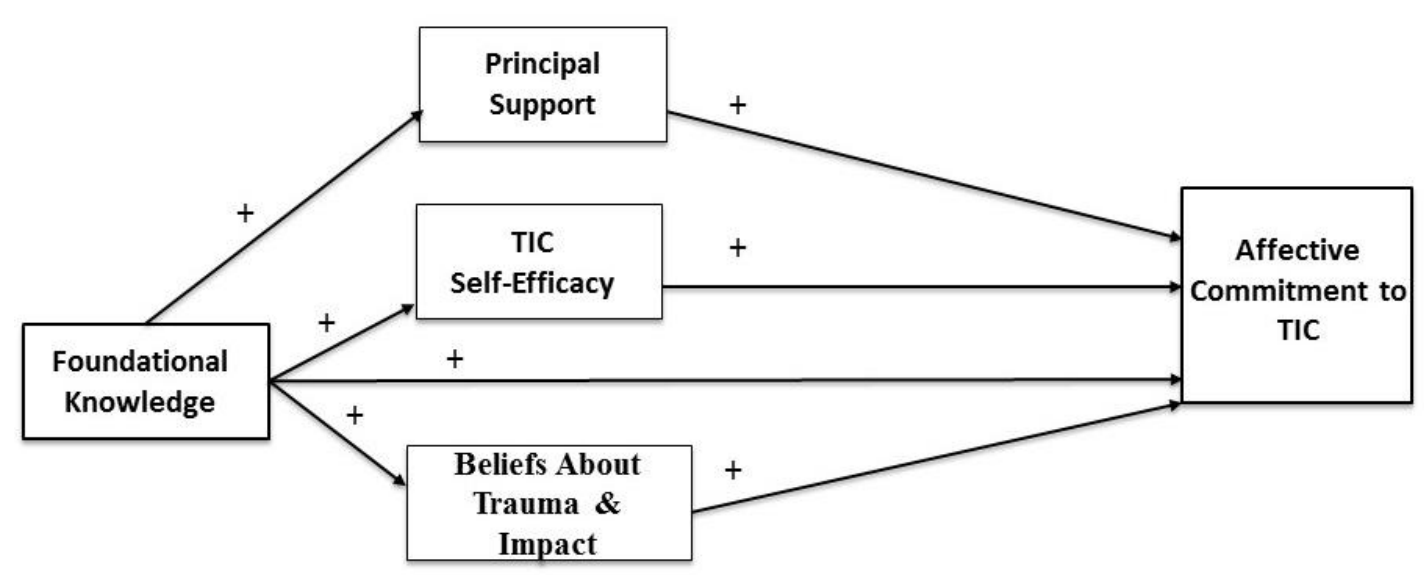

Figure 9. Indirect paths from foundational knowledge to affective commitment to trauma-informed care

In order to determine whether the mediated effect was partial, complete, or nonexistent, the direct effect of knowledge on affective commitment was observed in a saturated model without the mediators (principal support, TIC self-efficacy, and beliefs about trauma) included. See Table 7 for a summary. Once the direct effect was noted, the mediators were added back into the model one at a time. As shown in Table 7, the relationship between knowledge and affective commitment dropped in strength once the mediators were added into the model, yet it remained significant. This suggested a partially mediated relationship (MacKinnon, 2008) whereby knowledge still explained unique variance in affective commitment beyond the indirect effects.

The bootstrapped significance test for indirect effects showed a significant mediation of knowledge on affective commitment through all three of these variables. Self-efficacy 
and beliefs showed the strongest effects, $p=.003$ and $p=.001$, respectively. However, the indirect effect through principal support (PrinSupp) was also significant, $p=.020$.

This analysis supported mediation through a single mediator. However, because knowledge had three mediated relationships with affective commitment, there was also a combined effect.

Table 7.

Standardized Direct and Indirect Effects between Knowledge and Affective Commitment

\begin{tabular}{cccc}
\hline Relationship & $\begin{array}{c}\text { Direct Effect } \\
\text { Without } \\
\text { Mediators }\end{array}$ & $\begin{array}{c}\text { Direct Effect } \\
\text { With } \\
\text { Mediator }\end{array}$ & $\begin{array}{c}\text { Sig } \\
\text { Indirect } \\
\text { Effect }\end{array}$ \\
\hline Knowledge $\rightarrow$ PrinSupp $\rightarrow$ Affective & $.267^{* *}$ & $.260^{* *}$ & $p=.020$ \\
Knowledge $\rightarrow$ SelfEff $\rightarrow$ Affective & $.267^{* *}$ & $.258^{* * *}$ & $p=.003$ \\
Knowledge $\rightarrow$ Beliefs $\rightarrow$ Affective & $.267^{* *}$ & $.258^{* *}$ & $p=.001$
\end{tabular}

Note: PrinSupp = principal support. Affective $=$ affective commitment to TIC. SelfEff $=$ self-efficacy. In this test of the indirect effects through knowledge, the direct effects between PrinSupp, SelfEff, and Beliefs with affective commitment were retained. Indirect effects were added one at a time.

$* \mathrm{p}<.05 * * \mathrm{p}<.01 * * * \mathrm{P}<.001$

Table 8 provides a complete summary of standardized and unstandardized estimates for the direct, indirect, and total effects in this model. 
Table 8.

Estimates of Direct and Indirect Effects of Final Model

Effects

b (SE)

$\beta(\mathrm{SE})$

$95 \%$ CI

$\beta$

\section{Direct effects}

\begin{tabular}{l}
\hline Knowledge $\rightarrow$ Affective $\left(c^{\prime}\right)$ \\
Knowledge $\rightarrow$ Beliefs $\left(a_{1}\right)$ \\
Knowledge $\rightarrow$ SelfEff $\left(a_{2}\right)$ \\
Knowledge $\rightarrow$ PrinSupp $\left(a_{3}\right)$ \\
Beliefs $\rightarrow$ Affective $\left(b_{1}\right)$ \\
SelfEff $\rightarrow$ Affective $\left(b_{2}\right)$ \\
PrinSupp $\rightarrow$ Affective $\left(b_{3}\right)$
\end{tabular}

$.124(.042) * *$

$.223(.075) * *$

$.081-.372$

$.181(.054) * * *$

$.367(.088) * *$

$.175-.527$

$.273(.047) * * *$

$.493(.076)^{* *}$

$.343-.636$

$.122(.054)^{*}$

$.242(.108) *$

$.030-.453$

$.357(.077)^{* *}$

$.316(.078)^{* *}$

.163-.460

$.235(.089)^{* *}$

$.234(.087) * *$

.074-.409

$.413(.077)^{* * *}$

$.375(.063) * * *$

$.249-.502$

\section{Indirect Effects}

Knowledge $\rightarrow$ Affective $\left(a_{1} x b_{1}\right)^{\mathrm{a}}$
Knowledge $\rightarrow$ Affective $\left(a_{2} x b_{2}\right)^{\mathrm{a}}$
Knowledge $\rightarrow$ Affective $\left(a_{3} x b_{3}\right)^{\mathrm{a}}$
Knowledge $\rightarrow$ Affective full model

\section{Total Effects}

\begin{tabular}{l} 
Knowledge $\rightarrow$ Affective $\left(c_{1}\right)^{\mathrm{b}}$ \\
Knowledge $\rightarrow$ Affective $\left(c_{2}\right)^{\mathrm{b}}$ \\
Knowledge $\rightarrow$ Affective $\left(c_{3}\right)^{\mathrm{b}}$ \\
Knowledge $\rightarrow$ Affective full model \\
\hline
\end{tabular}

$.065(.026) * * * \quad .116$

$.064(.028)^{* *} \quad .115$

$.051(.026)^{*}$

.091

$.179(.048) * * *$

$.322(.068) * * *$

$.191-.447$

\section{Covariance}

\section{Err1 $\leftrightarrow$ Err2}

.189

.339

.188

.338

.175

.314

$.304(.063)^{* * *}$

$.545(.090)^{* * *}$

$.349-.689$

Err2 $\leftrightarrow$ Err3

$16.436(4.22)$

$.365^{* *} *$

$.190-.529$

13.608 (3.83)

$.324 *$

.101-.489

Note: $b=$ unstandardized estimates, $\beta=$ standardized estimates. Affective $=$ affective commitment to TIC. SelfEff $=$ self-efficacy . PrinSupp $=$ principal support. The specific indirect effects were calculated using a user-defined AxB estimand in AMOS created by Gaskin (2016). These represent unstandardized estimates.

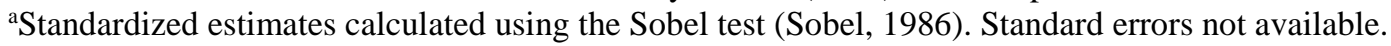

${ }^{\mathrm{b}}$ Standard errors were not available for specific total effects. AMOS provides standard errors for the full model only.

$* \mathrm{p}<.05 * * \mathrm{p}<.01 * * * \mathrm{P}<.001$ 
Bootstrap bias-controlled standard errors and confidence intervals (95\%) are provided for the standardized estimates. All indirect effects were significant and approximately equal in strength, with the path through beliefs being the strongest $(p<.001)$ and the path through principal support being the weakest $(p<.05)$. The total mediated effect from knowledge to affective commitment (through all pathways together) was significant ( $\beta$ $=.322, p<.001)$ and accounted for $10 \%$ of the variance in affective commitment.

\section{Hypothesis 5: Organizational strain will moderate the relationship between the} mediating variables and affective commitment to TIC. It was hypothesized that organizational strain would moderate the relationship between affective commitment to TIC and perceived principal support, self-efficacy, and beliefs about trauma. Moderation can be evident in the presence of interactions between single variables, or it can result in group differences across the model.

Moderated mediation. Organizational strain did not contribute to the hypothesized original model and was removed for the re-specified version. Therefore, it was unlikely that interactions would have an impact. However, to be certain, interaction terms were created and added to the model one at a time along with a direct effect from organizational strain to affective commitment. As predicted, the interactive effects of this variable on beliefs about trauma, TIC self-efficacy, and perception of principal support (peers and leadership) of TIC resulted in poor fit at the model level. Further, estimates and $p$ values were small and nonsignificant, as shown in Table 9. 
Table 9.

Interaction Effects

\begin{tabular}{lccc}
\hline & $\beta$ & SE & $p$ \\
\hline Model 1 & & & \\
OrgStrain & .023 & .073 & .707 \\
OrgStrain x PrinSupp & -.033 & .071 & .685 \\
Model 2 & & & \\
OrgStrain & .016 & .070 & .776 \\
OrgStrain x SelfEff & -.021 & .077 & .806 \\
Model 3 & & & \\
OrgStrain & .013 & .056 & .744 \\
OrgStrain x Beliefs & .020 & .061 & .722
\end{tabular}

Note: Bootstrap estimates are provided with standardized standard errors and $\mathrm{p}$ values. OrgStrain $=$ organizational strain. PrinSupp $=$ principal support. SelfEff $=$ self-efficacy.

Multigroup comparison. As another method to test moderation, group differences were evaluated using a chi-square difference score. Organizational strain (OrgStrain) was recoded as binary with scale score values above 40 (more than mildly agree that there is stress and turbulence at the organization) being assigned to the high strain group (value of 1) and values equal to or below 40 (mildly agree or disagree that there is stress and turbulence at the organization) being assigned to the low strain group (value of 0). Each group contained 59 cases. Chi-square difference test indicated that at the model level, there was no difference between the high strain group and the low strain group, $\chi^{2}(7)=$ $9.178, p=.240$. Individual paths were also tested in order to determine specific differences between the high strain and low strain groups (see Table 10). Both the high 
strain and the low strain groups had significant paths and nonsignificant paths, which differed by group.

Table 10

Standardized Estimates Compared Across High and Low Organizational Strain

\begin{tabular}{|c|c|c|c|}
\hline & $\beta$ & $\mathrm{SE}$ & $p$ \\
\hline \multicolumn{4}{|l|}{ High OrgStrain } \\
\hline Knowledge $\rightarrow$ PrinSupp & .415 & .149 & .015 \\
\hline Knowledge $\rightarrow$ SelfEff & .489 & .110 & .002 \\
\hline Knowledge $\rightarrow$ Beliefs & .415 & .117 & .003 \\
\hline Beliefs $\rightarrow$ Affective & .387 & .129 & .006 \\
\hline Knowledge $\rightarrow$ Affective & .219 & .124 & .070 \\
\hline SelfEff $\rightarrow$ Affective & .151 & .120 & .146 \\
\hline PrinSupp $\rightarrow$ Affective & .362 & .080 & .001 \\
\hline \multicolumn{4}{|l|}{ Low OrgStrain } \\
\hline Knowledge $\rightarrow$ PrinSupp & .077 & .118 & .458 \\
\hline Knowledge $\rightarrow$ SelfEff & .522 & .097 & .002 \\
\hline Knowledge $\rightarrow$ Beliefs & .299 & .149 & .065 \\
\hline Beliefs $\rightarrow$ Affective & .201 & .110 & .108 \\
\hline Knowledge $\rightarrow$ Affective & .203 & .110 & .047 \\
\hline SelfEff $\rightarrow$ Affective & .387 & .123 & .005 \\
\hline PrinSupp $\rightarrow$ Affective & .325 & .110 & .006 \\
\hline
\end{tabular}


For the high strain group, knowledge did not uniquely predict affective commitment ( $\beta$ $=.219, p=.070)$ nor did TIC self-efficacy predict affective commitment $(\beta=.151, p=$ .146). For the high strain group, the most significant predictor (as a direct effect) was the path between principal support and affective commitment $(\beta=.362, p=.001)$. For the low strain group, knowledge did not predict principal support $(\beta=.077, p=.458)$ nor did it predict beliefs ( $\beta=.299, p=.065)$. Additionally, for the low strain group, beliefs did not significantly predict affective commitment ( $\beta=.201, p=.108)$. For the low strain group, the most significant predictor (as a direct effect) was the path between knowledge and self-efficacy $(\beta=.522, p=.002)$. For the low strain group, the most significant predictor of commitment was TIC self-efficacy $(\beta=.387, p=.005)$.

Despite these individual group effects, results from chi-square difference analysis suggested that there were no significant differences between the high strain group and the low strain group on individual paths at $\mathrm{p}<.05$ (see Table 11). However, two paths were close to being significantly different between these two groups. The difference between groups for the path between beliefs and affective commitment neared significance, $\mathrm{p}=.057$. Specifically, the direct effect between beliefs and affective commitment was stronger for the high strain group $(\beta=.387)$ than it was for the low strain group $(\beta=.201)$, suggesting that under high strain conditions, TIC congruent beliefs lead to greater commitment to TIC. Perhaps in organizations with high strain, the TIC congruent beliefs create a sense of urgency, which in turn increases commitment. For the low strain group, 
TIC congruent beliefs about trauma did not significantly predict affective commitment to TIC.

Table 11.

Individual Path Comparisons between High Strain and Low Strain Groups

\begin{tabular}{lccc}
\hline & Chi-Square & df & $p$ value \\
\hline Knowledge $\rightarrow$ PrinSupp & 3.347 & 1 & .067 \\
Knowledge $\rightarrow$ SelfEff & .032 & 1 & .859 \\
Knowledge $\rightarrow$ Beliefs & .182 & 1 & .669 \\
Beliefs $\rightarrow$ Affective & 3.623 & 1 & .057 \\
Knowledge $\rightarrow$ Affective & .074 & 1 & .785 \\
SelfEff $\rightarrow$ Affective & 2.060 & 1 & .151 \\
PrinSupp $\rightarrow$ Affective & .438 & 1 & .508
\end{tabular}

Note: $\chi^{2}=$ chi square. $\mathrm{df}=$ degrees of freedom. Affective $=$ affective commitment to TIC. Paths were constrained one at a time to test significance.

The other path that neared significance on a chi-square difference test was the path between knowledge and principal support $(p=.067)$. The direct effect between knowledge and principal support was stronger for the high strain group $(\beta=.415)$ than for the low strain group $(\beta=.077)$. This difference suggested that under conditions of higher strain, an increase in knowledge leads to a more significant increase in perceived principal support. Likewise, under conditions of lower strain, an increase in knowledge does not significantly predict an increase in perceived principal support. Caution should be exercised when interpreting this finding, however, as the difference between real and perceived principal support could be highlighting a spurious relationship. Under 
conditions of high strain, peers and leadership may be more supportive of TIC which has little to do with an individual's knowledge. Instead it could be a group condition. This variable may be more appropriately considered at the organizational (group) level than the individual level.

Other group comparisons. As part of preliminary data analysis, group comparisons were also conducted using prior training and years of experience as grouping variables in order to better explain the data. Group differences were analyzed for all five variables. Results related to knowledge, beliefs, and self-efficacy were particularly important because of the possible influence on these variables from prior training and experience.

Years of experience. An analysis of variance (ANOVA) was performed between three groups to explore group differences based on years of experience. The high group included participants with 10 or more years in the field. The medium group included participants who had between 6-10 years of experience in the field, and the low group were those individuals with five or fewer years in the field.

Nonnormality in the data was not a concern given the group sizes of approximately 30 (Pallant, 2010). Levene's test for homogeneity of variance was not significant ( $p>.05$ ) for any variable, with the exception of affective commitment. In this case, the Levene's statistic was significant ( $\mathrm{p}=.003)$; therefore, the Brown-Forsythe test was used.

None of the variables showed a significant difference based on years of experience although affective commitment trended toward significance, $(p=.06)$. For all variables, those individuals with less experience had higher scores on the variables than those with 
more experience. For example, individuals with less experience showed greater TIC related self-efficacy $(M=39.26)$ than individuals with the most experience $(M=36.90)$. This relationship held across all variables, with the exception of knowledge in which case the medium experience group showed the most knowledge followed closely by the low experience group. See Appendix C, Table C3 for a summary of the data.

Prior training and other exposure. Group differences were also evaluated based on prior training/exposure. A sum score was created from the amount of previous face-toface training, webinars, and books, with a median equal to 7.0. A median split was done in order to create two groups, a high training group and a low training group.

All of the variables with the exception of principal support showed a significant difference based on prior training. Similar to the group findings with years of experience, those individuals who had more training exposure had lower scores on the variables than those who had less training. For example, individuals with less prior training exposure had more knowledge $(\mathrm{M}=106.25)$ than individuals who had more training exposure $(\mathrm{M}=87.31)$. This pattern was consistent across all five variables. See Appendix $\mathrm{C}$, Table C5 for a summary of the data. 


\section{Chapter 6}

\section{Discussion}

The purpose of this study was to explore the factors that facilitate affective commitment to TIC. Many interesting findings emerged from this research. Following are some of the key results:

- Foundational knowledge predicted commitment to TIC.

- Perceived principal support from one's organization, TIC self-efficacy, and beliefs about trauma also significantly predicted commitment to TIC.

- Foundational knowledge predicted commitment to TIC through perceived principal support from the organization, TIC self-efficacy, and beliefs about trauma (partially mediated pathways).

- Organizational strain did not predict commitment to TIC nor did it moderate the effects of the other variables in the model.

- Organizational strain, as a grouping variable (high versus low) produced different effects on the other variables included in the model.

- Among individuals perceiving high strain, commitment was not predicted by foundational knowledge.

- The strongest predictor of commitment to TIC among individuals perceiving high strain in the organization was principal support.

- Among individuals perceiving low strain, commitment was not predicted 
by beliefs about trauma.

○ The strongest predictor of commitment to TIC among individuals perceiving low strain in the organization was TIC self-efficacy.

Following, is a discussion of the findings and considerations for future research.

\section{Study Findings}

Affective commitment to trauma-informed care. Affective commitment represents favorable attitude and buy-in (Herscovitch \& Meyer 2002; Meyer et al., 2007) and often precedes a change in behavior (Ajzen \& Fishbein, 2005; Fedor et al., 2006).

Overall, participants expressed a high level of affective commitment toward TIC, moderately to strongly suggesting that TIC was a good idea for their organization. Only 9\% disagreed or were not sure. Strong support for TIC is not surprising, considering that respondents represented the fields of mental health, public health, and early childhood. Mental health providers routinely work with trauma survivors and have a first-hand knowledge of the impact of trauma (Najavits \& Hien, 2013; Savage et al., 2007). Public health, with its attention to social determinants of health (Koh et. al., 2010; Williams, Costa, Odunlami, \& Mohammed, 2008), has a keen awareness of the impact of social and environmental conditions (World Health Organization, 2014). Additionally, this group offers many services to individuals experiencing life challenges, bringing them into regular contact with the impact of adversity (see Muskett, 2014, for a review). Moreover, the early education field, with the clarion call about the importance of the first years of life, is blazing the trail for tremendous research and intervention work highlighting the 
profound impact of adversity during the developmental stages of life (Shonkoff et.al., 2012).

All variables, with the exception of organizational strain, significantly predicted affective commitment to TIC. Understanding these facilitating factors will aid in TIC implementation especially for individuals experiencing weaker commitment.

Knowledge about trauma-informed care. Knowledge about TIC was the primary predictor in this study as much of the literature suggests that acquiring foundational knowledge is the first step in becoming trauma-informed (Fallot \& Harris, 2006, 2009; Guarino et al., 2009; Hopper et al., 2010; Lebel \& Kelly, 2014; SAMHSA, 2014b). VanDeusen-Lukas and colleagues (2008) noted that implementer knowledge is one of the most important predictors of success.

Across six topics, respondents indicated that it was somewhat to completely true that they understood and could explain the content related to TIC. This finding is encouraging yet perplexing given that, in this sample, those with the greatest amount of knowledge represented the group with the least amount of previous training and the least amount of experience in the field (less than 5 years). Overall, the sample reported relatively little previous training. Training through webinars was the most common format for information acquisition, and respondents indicated that they had some prior training through this method. Through in-person training and training by books, respondents indicated very little prior experience. What explains the inverse relationship between prior training and knowledge? 
Several possibilities might justify this finding. It is common for individuals to overestimate their knowledge (Sitzmann et al., 2010), which could be the case for those individuals endorsing knowledge about TIC despite a small amount of prior training. Alternatively, those individuals with more training endorsed less knowledge. It could be that the individuals with more training and exposure are more aware of the knowledge they lack. This reflects what is known as the response shift bias (Howard \& Dailey, 1979) in which an individual essentially doesn't know what he or she doesn't know until after he or she has completed a training, class, or intervention. According to Pratt, McGuigan, and Katzev (2000), "This bias can be defined as a program-produced change in the participants' understanding of the construct being measured" (p. 342). This phenomenon is well established in the literature, calling into question pretests of knowledge (Nimon, Zigarmi, \& Allen, 2011; Schwartz \& Sprangers, 2010).

However, it could also be that the general attention toward adversity and the struggles facing trauma survivors within these fields has provided experience-based foundational knowledge that maps onto the knowledge topics in this study. In terms of specific information, it was interesting to note that historical trauma and oppression was the subject area about which respondents felt most knowledgeable. This may reflect the overlap that exists between TIC and other social awareness efforts that have taken hold across many of these disciplines, e.g., cultural competence and equity and inclusion. Respondents also felt knowledgeable about the neurobiology of trauma, a topic area that is often included in trainings and webinars. Respondents felt least knowledgeable about 
the principles of TIC, which is a surprising finding given their high level of TIC-related self-efficacy. This inconsistency could simply reflect that the self-efficacy questions were more abstract (e.g., I have the skills that are needed to make Trauma-Informed Care work) whereas the knowledge questions were more clear-cut and exhaustive (e.g., I know the principles of $T I C)$.

Knowledge was also greater among newer staff (those with fewer than five years in the field; $M=97.47$ ) compared to those who had 10 or more years of experience $(\mathrm{M}=95.76)$ although the difference was not statistically significant. TIC is a relatively new focus in the human services field garnering considerable attention in recent years (Butler \& Wolf, 2009; Reeves, 2015). Therefore, the higher levels of perceived knowledge, self-efficacy, beliefs, and affective commitment among newer staff (as compared to more veteran staff) could be a result of this increased exposure to the topic. In many states, an awareness and focus on TIC is being incorporated into state policy ${ }^{6}$. TIC is also being incorporated into educational programs (CSWE, 2008, 2012; Layne et al., 2011). As a result, it is reasonable that individuals new to the workforce would already have knowledge about and exposure to TIC. Future research should focus on the specific knowledge most predictive of successful TIC implementation. Further, because self-reported knowledge is often found to be unrelated to tested factual knowledge (Ladwig, Dalrymple, Brossard, Scheufele, \& Corley, 2012), it will be important to

\footnotetext{
${ }^{6}$ See Oregon Health Authority's policy on trauma-informed services, http://www.oregon.gov/oha/amh/trauma-policy/Trauma\%20Policy.pdf, for an example.
} 
understand if perceived knowledge is enough for someone to feel competent to apply TIC knowledge to practice.

Knowledge predicting affective commitment to TIC. As anticipated, foundational knowledge significantly predicted affective commitment to $\operatorname{TIC}(\beta=.22, p<.010)$. In addition, foundational knowledge indirectly predicted commitment through an increase in TIC-related self-efficacy, an increase in TIC congruent beliefs about trauma and its impact, and an increase in perceived principal support from peers and leadership.

Mediated path through principal support. The indirect effect from knowledge to affective commitment through principal support is a surprising finding, albeit the weakest of the mediated pathways. Included in this mediated path are a direct effect from knowledge to principal support and a direct effect from principal support to affective commitment.

Direct effect between principal support and affective commitment. A significant relationship between principal support and affective commitment was anticipated and turned out to be one of the strongest direct effects in the model $(\beta=.39, p<.001)$. In fact, a one-unit increase in principal support corresponded with a nearly $50 \%$ increase in affective commitment. Consistent with other findings in the literature recognizing the importance of leadership support (Esaki, Hopson, \& Middleton, 2014; Liu et al., 2012; VanDeusen-Lukas et al., 2008), this finding adds empirical support for the importance of principal support as it relates to TIC. Hendricks et al. (2011) noted that leadership 
commitment to TIC facilitates implementation. For organizations struggling to put TIC into practice, identifying leadership champions may be the answer.

Direct effect between knowledge and principal support. The surprising finding involved in the indirect effect through principal support was the direct connection between foundational knowledge and principal support. It was originally hypothesized that there would be no significant relationship between these two variables. Contrary to expectations, this direct effect was found to be significant $(\beta=.24, p<.05)$, and knowledge explained approximately $6 \%$ of the variance in principal support. While leadership buy-in and commitment is a recommended necessary condition for TIC (see Fallot \& Harris, 2006, 2009; Harris \& Fallot, 2001; Hendricks et al., 2011), it is less clear how an increase in foundational knowledge directly relates to an increase in perceived principal support (from coworkers and leadership). Perhaps an increase in knowledge leads an individual to simply be more aware of coworker and leadership support, reflecting a correlated but not causal relationship. However, there were no group differences in perceived principal support based on prior training or years of experience. An increase in perceived principal support could also reflect an actual increase in demonstrated support if coworkers and leadership are, themselves, becoming more knowledgeable about TIC. Although perceived principal support was measured at the individual level in the current study, it may be informative to investigate this construct at the work group or organizational level (see Limitations). It is well known in the organizational literature that work environments can create collective beliefs and 
attitudes, known as emotional contagion (McCoach, Gable, \& Madura, 2013;

Vijayalakshmi \& Bhattacharyya, 2012), which in turn can create organizational culture and climate (see Impact of Organizational Culture and Climate, below).

Mediated path through self-efficacy. Another significant indirect path from knowledge to affective commitment was mediated through TIC self-efficacy, indicating that some of the effect that knowledge has on affective commitment is created through an increase in TIC self-efficacy. This is not a surprising finding given that self-efficacy is an important indicator of change readiness (Armenakis et al., 2007).

Direct effect between self-efficacy and affective commitment. It is not surprising that an increase in self-efficacy corresponded to an increase in affective commitment, as this is well documented in the change literature (Damschroder et al., 2009; Herold et al., 2007; Herscovitch \& Meyer, 2002; Santhidran et al., 2013). Self-efficacy reflects a belief that we can successfully accomplish the requirements of a change effort (Damschroder et al., 2009). According to the Theory of Planned Behavior (see Ajzen, 1991), self-efficacy is a key component in the perceived behavioral control construct that predicts behavioral change. The direct effect from self-efficacy to affective commitment was strong ( $\beta=.23, p$ $<.01$ ), explaining $5 \%$ of the variance in commitment. The strength of this indirect effect, however, is primarily due to the strong relationship between knowledge and self-efficacy $(\beta=.49, p<.001)$.

Direct effect between knowledge and self-efficacy. Foundational knowledge and selfefficacy were strongly correlated, $(\mathrm{r}=.564)$ with a one-unit increase in knowledge leading 
to a .27-unit increase in self-efficacy. Leadership adoption is necessary for the innovation to be supported; however, individuals within the organization must also believe the new practice will be easy to implement and that it will add value (Solomons \& Spross, 2011).

Mediated path through beliefs. The final, and strongest, indirect pathway from knowledge to affective commitment that was considered in this model was mediated by beliefs about trauma. Beliefs and attitudes can be influential when adopting a traumainformed approach, as they have been shown to be predictors of behavior change (Ajzen, 2011; Ajzen \& Fishbein, 2005). For most professionals working in social services, positive attitudes including empathy, tolerance, and understanding are expectations of their service commitment (Gerdes \& Segal, 2011; Kinman \& Grant, 2011). However, little is known about how beliefs about trauma impact commitment to TIC.

In this sample, respondents moderately to strongly agreed that trauma is prevalent $(M=6.14)$ and can be connected to behavior and poor life outcomes $(M=6.60)$. They also mildly to moderately agreed that seeking and receiving services can be re-traumatizing $(M=5.53)$. However, when asked specifically about their own organization's programs and services causing trauma, they were less likely to agree with these statements (M=4.22). Is the organization with which these employees are affiliated delivering services in a trauma-informed manner, or are these employees more biased about their organization's ability to re-traumatize service recipients? According to the selfenhancement bias, it is common for individuals to have inflated and overestimated evaluations of their own performance and abilities (Alicke \& Sedikides, 2009; Sedikides 
\& Gregg, 2008) as a source of self-protection. Are these service providers biased about their organization's potential for re-traumatization? It can be that "such biases can obscure needed areas of improvement" (Neff, 2011, p. 2). Service recipient input about organizational impact and performance is central to delivering TIC (Elliott et al., 2005; Fallot \& Harris, 2009; SAMHSA, 2014b) and will provide needed feedback on this matter.

Direct effect between beliefs and affective commitment. In the current study, beliefs and affective commitment were strongly correlated $(\mathrm{r}=.576)$. This finding is logical given that the beliefs addressed in this study reflect core tenets associated with a traumainformed approach (Elliott et al., 2005; Fallot \& Harris, 2006, 2009; Hopper et al., 2010; Ko et al., 2008; SAMHSA, 2012, 2014b). Beyond being simply related, beliefs significantly predicted affective commitment, explaining $10 \%$ of its variance.

Direct effect between knowledge and beliefs. In the current study, knowledge significantly predicted beliefs, explaining 13\% of its variance. Courtois and Gold (2009) suggested that perhaps the solution to creating trauma sensitive beliefs rests entirely with more education.

As TIC takes hold across other disciplines that aren't necessarily aligned with the beliefs and attitudes most supportive of trauma survivors, it will be important to better understand the beliefs about trauma that support implementation of TIC.

Individually, the effects of these indirect paths are small; however, in combination, they account for approximately $10 \%$ of the variance of affective commitment. Even more 
important than the individual relationships and effects is the impact that all of the variables have on affective commitment. Taken as a whole, this model accounts for $65 \%$ of the variance in affective commitment. This finding suggests that in addition to providing foundational knowledge, as organizations move toward TIC implementation, they would be wise to address issues and concerns related to TIC self-efficacy, beliefs about trauma, and the involvement and support of peers and leadership.

Organizational strain moderating variables in model. The final consideration in this study was the effect that organizational strain (a combination of stress and change known as turbulence) had on the variables in the model. It was hypothesized that organizational strain would moderate the relationships between affective commitment and principal support, beliefs about trauma, and TIC self-efficacy. It is well known in the literature that organizational stress or turbulence can negatively impact employee willingness for innovation and change (Kulkarni et al., 2013). According to Herold et al. (2007), “An environment of pervasive change may negatively influence individuals' commitment to a given change, especially for those with low efficacy in dealing with change" (p. 948). The context in which the change is occurring appears to have a significant impact on the commitment to change (Coffman, 2007). Therefore, it is reasonable that organizational stress or turbulence would impact staff commitment to TIC (Vakola \& Nikolaou, 2005). Although logical, the findings did not support this hypothesis. 
Interactive effects of organizational strain. First, organizational strain was not related to affective commitment and contributed to a poor fit with the model. As a result, the variable was removed from SEM analysis. Although surprising, this finding may indicate that TIC resonates with staff for different reasons. Perhaps when organizational strain is high, employees believe TIC is needed. Alternatively, when strain is low, employees are willing and able to consider a new change effort such as TIC. Given both of these situations, rather than concluding that strain has no effect, it may be true that strain is affecting individuals in opposite ways, cancelling out any overall group effect. Bloom and Farragher (2011) recognized the profound impact of organizational stress and its resulting ripple effect. Despite confusing results in the current study, organizational strain is an important consideration, especially in terms of implementation and sustainability of TIC (Bloom \& Farragher, 2011, 2013).

Grouping effects of organizational strain. In the current study, differences between the group experiencing high strain and the group experiencing low strain was more informative. Although the overall fit of the model was not significantly different between the high strain and low strain groups, some individual path differences trended toward significant.

Significant path differences related to organizational strain. The difference between the direct effect of beliefs and affective commitment for high strain and low strain was nearly significant, $\chi^{2}(1)=3.62, p=.057$, with this path being stronger for the high strain group $(\beta=.387)$ than for the low strain group $(\beta=.201)$. This suggests that TIC congruent 
beliefs about trauma lead to greater commitment to TIC when organizational strain is perceived to be high as opposed to low. For the low strain group, TIC congruent beliefs about trauma did not significantly predict their affective commitment to TIC. As mentioned, this group difference could reflect staff's interest in TIC given high strain conditions. Perhaps in this situation, the TIC congruent beliefs compel individuals to commit to a trauma-informed approach. In the low strain condition, individuals may have TIC congruent beliefs but they do not feel as compelled to engage in something new.

The other path that nears significance on a chi-square difference test is the path between knowledge and principal support $(p=.067)$. The direct effect between knowledge and principal support was stronger for the high strain group $(\beta=.415)$ than it was for the low strain group $(\beta=.077)$. This difference suggests that under conditions of higher strain, an increase in knowledge leads to a more significant increase in perceived principal support. Likewise, under conditions of lower strain, an increase in knowledge does not significantly predict an increase in perceived principal support. It could be that under strain, employees look for support from peers and leadership differently than they do if they are not feeling stress. Again, caution should be exercised when interpreting this finding as the difference between real and perceived principal support could be highlighting a spurious relationship. It could be that under conditions of high strain, peers and leadership are actually more supportive of TIC and that it has little to do with an individual's knowledge, reflecting a group condition instead. 
The most significant predictor of commitment to TIC among the group experiencing high strain was perceived principal support from peers and leadership. The most significant predictor of commitment to TIC among the group experiencing low strain was TIC self-efficacy.

Nonsignificant paths as a result of organizational strain. Several paths were no longer significant when looking at high or low strain only. For instance, the path between knowledge and affective commitment was not significant in the high strain group $(p=.070)$, nor was the path from self-efficacy to affective commitment $(p=.146)$. Both of these paths were significant at $p<.01$ level when the full sample was considered. This finding suggests that individuals who have a sense of high strain in the organization will not be committed to TIC despite increased knowledge about the topic or the feeling of self-efficacy. Herold et al. (2007) found high turbulence related to low commitment. In organizations experiencing high strain, it may be necessary to first address the stress before introducing TIC.

In the low strain group, the paths between knowledge and principal support, and knowledge and beliefs were not significant, despite being significant in the larger sample. In addition, the path from beliefs to affective commitment was no longer significant. This finding suggests that when organizational stress and turbulence is perceived as low, foundational knowledge about TIC does not change staff beliefs about trauma and its impact nor does it change their perception of principal support for TIC. Additionally, individuals are not more likely to be committed to TIC because their beliefs are 
congruent with TIC. Perhaps this represents a status quo perspective whereby employees in low stress environments do not feel the need to alter their beliefs or their commitment to new initiatives because everything is working fine.

These findings are a reminder that organizational conditions, such as stress and turbulence, can have an impact on change readiness and implementation.

\section{Considerations}

Impact of organizational culture and climate. In addition to organizational strain, the other variables in this model are likely reflecting organizational dynamics. Social conditions can support or derail implementation; therefore, the organization level is an area in need of focus (Bloom \& Farragher, 2011). Bloom and colleagues have long understood the impact that organizational functioning can have on staff and the delivery of services (Bloom, 2006, 2010; Bloom \& Farragher, 2011, 2013). As a result of a parallel process, organizational stress can create institutional environments that start to mirror the trauma seen among the consumers seeking services, jeopardizing traumainformed service delivery (Bloom, 2006, 2010; Bloom \& Farragher, 2011, 2013).

Glisson (2007) noted that work environments, characterized as organizational climate, can be important in influencing staff function and morale as well as the successful implementation of services. Both organizational culture (the way things are done) and organizational climate (the way people perceive their work environment) seem to impact morale, job satisfaction, and staff turnover. Additionally, both influence the social context including attitudes, beliefs, norms, and values. Moreover, culture and climate 
impact employee attitudes toward change (Choi, 2011) and the success of implementation (Damschroder et al., 2009). The majority of respondents in the current study (79\%) worked for the same agency, albeit a large organization across multiple locations. It could be that there is a shared culture impacting these data. The effect of organizational climate and culture is not well understood, especially as it relates to trauma-informed care implementation. This will be an important next step for research.

Knowledge translation. Knowledge translation (KT) represents the quick movement from knowledge to application (Mackay, Petermann, Hurrell, \& Hodgins, 2015). These authors noted, "Knowledge translation is about closing the gap between what we know and what we do" (Mackay et al., 2015, p. 180). A growing field, KT represents the "synthesis, dissemination, exchange and ethically sound application of knowledge" (Paterson, Lagosky, \& Mason, 2016, p. 2).

This study presents an opportunity to think about KT from two perspectives. First, given the sparse number of empirical studies related to TIC implementation (Esaki et al., 2014; Kramer et al., 2013; Kusmaul et al., 2015; Wolf et al., 2014), KT is an important consideration as researchers ensure that overall findings are being translated into practice for the field? Second, KT can inform practitioners who are eager to convert TIC-related foundational knowledge into practical skills. Understanding how foundational knowledge relates to action will help support the change effort (VanDeusen-Lukas et al., 2008) and move organizations toward implementation of TIC. In the current study, $61 \%$ of respondents moderately to strongly agreed that they had the skills needed for TIC and 
$50 \%$ felt that they had the knowledge needed. Unfortunately, translating research into action can take years (Morris, Wooding, \& Grant, 2011). Too often, “new knowledge does not get translated into action or does so only after a lengthy lag time" (Goldner, Jenkins, \& Fischer, 2014, p. 161). Educational outreach, use of opinion leaders, and interactive educational meetings are among the strategies to increase knowledge to practice. Goldner, Jenkins, and Fischer (2013) noted that KT strategies are most successful when the information is simple and tools are available to support application. Strong leadership and willingness within the organization are two important criteria for effective KT. Among respondents of this study, $16 \%$ disagreed that they had the needed skills and $21 \%$ disagreed that they had the knowledge. What will help the uptake of knowledge for those staff not feeling adequately prepared for TIC?

\section{Next Steps}

This study presents a first step in identifying facilitating factors for staff commitment to TIC. However, refining the model is a possible next step, especially given that principal support and organizational strain resulted in surprising findings. It may be that organizational strain, in particular, will have more impact in other organizations. The role that principal support plays in the model also needs to be better understood. Is this an individual level variable or should it be considered at a work group or organizational level? Further, how is principal support affected by the other variables in the model? Direct effects between the mediating variables were not investigated in the current study but might be informative to the understanding of overall TIC readiness. 
Scale development is another possible next step. Both the knowledge and the beliefs measures were created for this study; therefore, there is opportunity for fine tuning these variables. Is perceived knowledge adequate in order to predict commitment to TIC and create a sense of self-efficacy or should actual knowledge be measured? Understanding the topics related to TIC that have the greatest impact would also be beneficial for training. With the goal of effectively translating TIC knowledge into practice, trainings could be tailored to maximize efficiency.

Although this study adds to the growing understanding of the factors affecting a commitment to TIC, more work is needed to understand how commitment relates to TIC implementation. The implementation process is "contingent and complex, predicated on what is changing, the make-up of leadership and contextual factors (internal and external) surrounding the change" (Caldwell, 2013, p. 21). Furthermore, change doesn't occur in a linear fashion, with only one change happening at a time (Langley, Smallman, Tsoukas, \& Van de ven, 2013). In fact, in most organizations, change is simultaneous and complex. As a result, individuals evaluate internal and external factors when considering change including the motivation for the change, the characteristics of leadership, and the organization's culture, climate, and commitment to resources, all variables important in creating individual readiness for change (Caldwell, 2013; Lehman et al., 2011). Continuing to explore the factors involved in a change toward TIC and the conditions on which these factors have an effect at the individual, workgroup, and organizational level will help aid TIC implementation. Fedor, Caldwell, and Herold (2006) claimed that the 
response to change is complex and must be considered at multiple levels:

"Operationalizing change simply in terms of its impact on either the individual or the work unit by itself is clearly not sufficient" (p. 20).

\section{Implications for Social Work}

With a focus on vulnerable and disadvantaged populations, social workers are well positioned to contribute to the knowledge base that will move TIC forward. Inherent in the social work code of ethics is a commitment to addressing social problems and helping those in need (National Association of Social Workers, 1999). No other discipline better understands the complexities of trauma and the critical need for trauma-informed care. Indeed, social work researchers commit to not only addressing these social problems but also to striving to improve policy, programming, and practice in an effort to create social change ${ }^{7}$. Marlowe and Adamson (2011) observed that social workers are able to integrate multiple perspectives, incorporating an understanding of cultural and environmental context as well as resilience and healing. This broad perspective will be needed as organizations work to implement a trauma-informed approach.

Ironically, despite a first-hand understanding of the impact of trauma, trauma education is not a standard component of social work education. Many scholars are recognizing the need for trauma education in social work programs, suggesting that this will aid in reducing workforce trauma (Adams \& Riggs, 2008; Bussey, 2008). Prevalence

\footnotetext{
${ }^{7}$ See Social Work Policy Institute, http://www.socialworkpolicy.org/about-the-social-work-policy-institute for more information.
} 
data suggest high levels of trauma exposure across most, if not all, of the fields of interest in social work, including child welfare, mental health, and addictions (Cohen \& Collens, 2013; CSWE, 2012; McKenzie-Mohr, 2004; Sprang, Craig, \& Clark, 2011). As a consequence, work-related trauma, including burnout, vicarious trauma, and secondary traumatic stress, is high among social workers (Bride et al., 2007; Newell \& MacNeil, 2010). Choi (2011) noted that, social workers will benefit from understanding the conditions that mediate the outcomes associated with work-related stress.

As a nascent field, research investigating TIC foundational knowledge and educational methods for acquiring knowledge is sparse (Strand, Abramovitz, Layne, Robinson, \& Way, 2014). To date, studies have primarily focused on methods of education delivery (Layne et al., 2011; Strand et al., 2014) and curriculum content (Layne et al., 2011). As one example, Layne and colleagues (2011) found that when using clinical vignettes, social work students showed "significant increases in self-efficacy in applying core trauma concepts to work with trauma exposed children and adolescents" (p. 249). Students indicated problem-based learning aided their ability to apply the knowledge to their work, an important element of KT.

The current study adds to the understanding of TIC knowledge and helps explain how knowledge relates to commitment to TIC, TIC self-efficacy, and beliefs about trauma. Social workers and social work researchers will be important to the ongoing research and will play an important role in this field moving forward. 


\section{Limitations}

This study contributes to the growing literature on trauma-informed care; however, there are limitations worth noting. Generalizing these findings to other populations is limited for a number of reasons. First, given the low response rate $(34 \%)$, the size of the sample was small. The participants were recruited from several trainings, one of which was earlier in the same year. The response rate from this particular training was only $17 \%$, suggesting sample recruitment would be more successful closer to the time of training. The other trainings resulted in a response rate of $44 \%$ overall, still lower than the average of $52.7 \%$ found in a meta-analysis of 500 studies but higher than the rate (36\%) typically found within organizations (Baruch \& Holtom, 2008).

Another limit to generalizability is that three-quarters or more of the participants $(73 \%)$ were female ${ }^{8}$. This was not surprising given the disproportionate number of females in the recruitment pool. Of the 420 invited to participate in the study, $87 \%$ $(n=364)$ were female, presenting a potential bias. How would these findings hold given a gender-balanced sample? Due to the difference in group size, a comparison by gender was not conducted.

Another significant limit to generalizability comes from the fact that $79 \%$ of the respondents work for the same organization. This organization is large $(n=252)$ with both public and mental health services, including substance abuse. Although there are many

\footnotetext{
${ }^{8}$ In response to the open-ended gender question, $73 \%$ responded female, $10 \%$ responded male, $17 \%$ did not respond.
} 
supervisors and managers overseeing services from numerous locations around the county, they all share a common director and possibly a similar organizational culture. The respondents also represent a similar geographical region and a limited number of disciplines (public and mental health). The impact of organizational culture is an important consideration and was included in the discussion section. Would these results be supported at another organization with a dissimilar climate and culture? Moreover, would these results be supported in other fields such as education or criminal justice or in other geographical locations ${ }^{9}$ ? Given the racial homogeneity of the county from which these data came, race was not included as a demographic question. It could be that there is a cross-racial difference in these variables. These are all important questions for future research, and they limit generalizability from the current study. Although the findings from this study cannot be assumed to transfer to different settings and organizations, these findings present reasonable considerations for organizations wishing to implement TIC.

The variables in this study were measured at the individual level, presenting another limitation to this research (Amis \& Aïssaoui, 2013). It is possible that workgroup or organizational influences are present. Differentiating individual versus collective beliefs and attitudes toward TIC, in the future, will provide greater clarity to findings.

\footnotetext{
${ }^{9}$ Deschutes County is a rural geographical area with 166,622 residents (US Census Bureau, 2011-2015), of whom $88 \%$ self-identify as white alone, not Hispanic or Latino.
} 
Admittedly, the current study contained referent-shifts (meaning that questions go from an "I" orientation such as I feel confident to an "other" orientation such as the top leaders support TIC). This shift in referent may have introduced confusion and inaccuracy. Rafferty, Jimmieson, and Armenakis (2013) noted that the predominant focus on individual factors of change readiness rather than workgroup and organization variables is problematic and represents a gap in the change readiness literature:

It is possible to imagine a situation where a particular individual has a high level of change readiness but is in a work group with low change readiness and works in an organization that also is characterized by low change readiness. In this situation, assessing individual readiness prior to change implementation is likely to result in an inaccurate understanding of the likelihood of change success ( $\mathrm{p}$. 129).

Another limitation is the influence the researcher may have had, indirectly, on the respondents. Many of the respondents had received training from the researcher. Known as leniency bias, there is a possibility that survey responses were affectively influenced because the respondents knew the researcher. Holding both positions, trainer and researcher, can introduce bias (Podsakoff et al., MacKenzie \& Podsakoff, 2012). For example, trainers might lead participants to learn, think, and respond in a way that somehow helps the research. To these points, the training was standardized and covered the foundational content that is presented at all TIC 101 or 201 trainings. The TIC principles are introduced, but very little is presented about implementation. The 
hypothesized factors involved in readiness were not mentioned at all during the foundational training. Moreover, the survey was voluntary and administered electronically. Potential participants were informed that the survey wouldn't be linked to their email address. Neither the organization nor the trainer knew who had participated.

Finally, there are always limitations related to survey data. For instance, respondents can be influenced by item characteristics including language choice, abstract or vague descriptions, use of common scale formats and anchors, and use of positive or negatively worded questions. The survey questions were piloted before distribution in an attempt to address clarity and social desirability of language. As far as the potential problems related to scale format and anchors, Podsakoff, MacKenzie, and Podsakoff (2012) admitted that there are potential issues but noted that using a consistent format (e.g., Likert) supports content validity. Pilot participants approved of the Likert format and answer choices.

\section{Conclusion}

The value of the current study comes from taking the model as a whole. It reinforces the understanding that organizations are complex and multifaceted and that any change effort must account for these multiple influences. If organizations get stuck implementing TIC, it might be more valuable to hone in on the perception of self-efficacy, beliefs about trauma, and perceived principal support rather than simply engaging in more training. It appears that individuals have the knowledge needed to commit to TIC (although a bit more emphasis on principles of TIC would probably be beneficial); therefore, focusing on building the skills and confidence as well as addressing underlying counter beliefs 
would be a worthwhile effort. As well, ensuring that organizational leadership support for TIC is visible across all levels of the organization should be a priority. Leaders should be modeling and championing the TIC concepts recognizing that this will filter down to direct service providers and other staff. Esaki et al. (2014) found that implementation success was related to support of leadership as leaders were the most likely to model TIC.

In organizations with high strain, it would be beneficial to the change effort to address the stress and turbulence as these factors relate to the commitment to TIC. Creating a culture of change readiness from leadership down can produce a contagion that influences individual staff readiness. 


\section{References}

Aarons, G. A. (2004). Mental health provider attitudes toward adoption of evidencebased practice: The Evidence-Based Practice Attitude Scale (EBPAS). Mental Health Services Research, 6(2), 61-74.

Adams, S. A., \& Riggs, S. A. (2008). An exploratory study of vicarious trauma among therapist trainees. Training and Education in Professional Psychology, 2(1), 26.

Ajzen, I. (2011). The theory of planned behavior: Reactions and reflections. Psychology and Health, 26(9), 1113-1127.

Ajzen, I., Czasch, C., \& Flood, M. G. (2009). From intentions to behavior: Implementation intention, commitment, and conscientiousness. Journal of Applied Social Psychology, 39(6), 1356-1372.

Ajzen, I., \& Fishbein, M. (2005). The influence of attitudes on behavior. The Handbook of Attitudes, 173, 221.

Alicke, M. D., \& Sedikides, C. (2009). Self-enhancement and self-protection: What they are and what they do. European Review of Social Psychology, 20(1), 1-48.

Alivernini, F., \& Lucidi, F. (2011). Relationship between social context, self-efficacy, motivation, academic achievement, and intention to drop out of high school: A longitudinal study. The Journal of Educational Research, 104(4), 241-252.

Amis, J. M., \& Aïssaoui, R. (2013). Readiness for change: An institutional perspective. Journal of Change Management, 13(1), 69-95. 
Appelbaum, S. H., Habashy, S., Malo, J. L., \& Shafiq, H. (2012). Back to the future: Revisiting Kotter's 1996 change model. Journal of Management Development, 31(8), 764-782.

Armenakis, A. A., Bernerth, J. B., Pitts, J. P., \& Walker, H. J. (2007). Organizational change recipients' beliefs scale development of an assessment instrument. The Journal of Applied Behavioral Science, 43(4), 481-505.

Armenakis, A. A., \& Harris, S. G. (2002). Crafting a change message to create transformational readiness. Journal of Organizational Change Management, 15(2), 169-183.

Armenakis, A. A., \& Harris, S. G. (2009). Reflections: Our journey in organizational change and research. Journal of Change Management, 9, 127-142.

Armenakis, A. A., Harris, S. G., \& Mossholder, K. W. (1993). Creating readiness for organizational change. Human Relations, 46(6), 681-703.

Baartman, L. K., \& de Bruijn, E. (2011). Integrating knowledge, skills and attitudes: Conceptualising learning processes towards vocational competence. Educational Research Review, 6(2), 125-134.

Baer, J. S., Wells, E. A., Rosengren, D. B., Hartzler, B., Beadnell, B., \& Dunn, C. (2009). Agency context and tailored training in technology transfer: A pilot evaluation of motivational interviewing training for community counselors. Journal of Substance Abuse Treatment, 37, 191-202. 
Baker, C. N., Brown, S. M., Wilcox, P. D., Overstreet, S., \& Arora, P. (2015).

Development and psychometric evaluation of the attitudes related to traumainformed care (ARTIC) scale. School Mental Health, 8(1), 61-76. doi:10.1007/s12310-015-9161-0.

Bandura, A. (1977). Self-efficacy: Toward a unifying theory of behavioral change. Psychological Review, 84(2), 191.

Bandura, A. (1986). Social foundations of thought and action: A social cognitive theory. Englewood Cliffs, NJ: Prentice-Hall.

Bandura, A. (1997). Self-efficacy: The exercise of control. New York, NY: Freeman.

Bandura, A. (2012). On the functional properties of perceived self-efficacy revisited. Journal of Management, 38(1), 9-44.

Baraldi, S., Kalyal, H. J., Berntson, E., Näswall, K., \& Sverke, M. (2010). The importance of commitment to change in public reform: An example from Pakistan. Journal of Change Management, 10(4), 347-368.

Barrett, J. H., Haslam, R. A., Lee, K. G., \& Ellis, M. J. (2005). Assessing attitudes and beliefs using the stage of change paradigm: Case study of health and safety appraisal within a manufacturing company. International Journal of Industrial Ergonomics, 35(10), 871-887. 
Bartunek, J. M., Rousseau, D. M., Rudolph, J. W., \& DePalma, J. A. (2006). On the receiving end: Sense-making, emotion, and assessments of an organizational change initiated by others. The Journal of Applied Behavioral Science, 42(2), 182-206.

Baruch, Y., \& Holtom, B. C. (2008). Survey response rate levels and trends in organizational research. Human Relations, 61(8), 1139-1160.

Bayraktar, S. (2011). Turkish preservice primary school teachers'science teaching, efficacy beliefs, and attitudes toward science: The effect of a primary teacher education program. School Science and Mathematics, 111(3), 83-92.

Beauchamp, M. R., Rhodes, R. E., Kreutzer, C., \& Rupert, J. L. (2011). Experiential versus genetic accounts of inactivity: Implications for inactive individuals' selfefficacy beliefs and intentions to exercise. Behavioral Medicine, 37(1), 8-14.

Bentler, P. M. (1990). Comparative fit indexes in structural models. Psychological Bulletin, 107(2), 238.

Black, M.C., Basile, K.C., Breiding, M.J., Smith, S.G., Walters, M.L., Merrick, M.T., Chen, J., \& Stevens, M.R. (2011). The national intimate partner and sexual violence survey: 2010 summary report. Atlanta, GA: National Center for Injury Prevention and Control, Centers for Disease Control and Prevention. Retrieved from https://www.cdc.gov/violenceprevention/nisvs/index.html. 
Blanch, A. (2003). Developing trauma-informed behavioral health systems. Alexandria, VA: National Association of State Mental Health Program Directors, National Technical Assistance Center for State Mental Health Planning.

Bloom, B. S., Englehart, M. D., Furst, E. J., Hill, W. H., \& Krathwohl, D. R. (Eds.) (1956). Taxonomy of educational objectives: The classification of educational goals, by a committee of college and university examiners. Handbook 1: Cognitive domain. New York, NY: David McKay.

Bloom, S. L. (1997). Creating sanctuary: Toward the evolution of sane societies. New York, NY: Routledge.

Bloom, S. L. (1999). Trauma theory abbreviated. Final action plan: A coordinated community-based response to family violence. Attorney General Mike Fisher's Task Force on Family Violence. Retrieved from http://www.sanctuaryweb.com/Publications/ListofPublications.aspx.

Bloom, S. L. (2006). Organizational stress as a barrier to trauma-sensitive change and system transformation. Alexandria, VA: National Technical Assistance Center for State Mental Health Planning Publications and Reports. Retrieved from http://www.sanctuaryweb.com/Publications/ListofPublications.aspx. 
Bloom, S. L. (2010). Organizational stress as a barrier to trauma informed service delivery. In M. Becker \& B. Levin (Eds.), B. A public health perspective of women's mental health (pp.295-311). New York, NY: Springer. Retrieved from https://ncfy.acf.hhs.gov/sites/default/files/docs/21360-

Organizational_Stress_as_a_Barrier_to_Trauma-Informed.pdf.

Bloom, S. \& Farragher, B. (2011). Destroying sanctuary: The crisis in human service delivery systems. New York, NY: Oxford University Press.

Bloom, S. L., \& Farragher, B. (2013). Restoring sanctuary: A new operating system for trauma-informed systems of care. New York, NY: Oxford University Press.

Bouckenooghe, D., Devos, G., \& Van den Broeck, H. (2009). Organizational change questionnaire: Climate of change, processes, and readiness: Development of a new instrument. The Journal of Psychology, 143(6), 559-599.

Bouckenooghe, D., Schwarz, G. M., \& Minbashian, A. (2015). Herscovitch and Meyer's three-component model of commitment to change: Meta-analytic findings. European Journal of Work and Organizational Psychology, 24(4), 578-595. doi:http://dx.doi.org.proxy.lib.pdx.edu/10.1080/1359432X.2014.963059

Boland, R. J., Lester, N. A., \& Williams, E. (2010). Writing multiple-choice questions. Academic Psychiatry, 34(4), 310. 
Brainerd, C. J., \& Reyna, V. F. (1990). Gist is the grist: Fuzzy-trace theory and the new intuitionism. Developmental Review, 10(1), 3-47.

Brainerd, C. J., \& Reyna, V. F. (2015). Memory and knowledge. Cognitive Modeling in Perception and Memory: A Festschrift for Richard M. Shiffrin, 173.

Bride, B. E., Radey, M., \& Figley, C. R. (2007). Measuring compassion fatigue. Clinical Social Work Journal, 35(3), 155-163.

Brossard, D., \& Shanahan, J. (2006). Do they know what they read? Building a scientific literacy measurement instrument based on science media coverage. Science Communication, 28(1), 47-63.

Brown, I. (2006). Nurses' attitudes towards adult patients who are obese: Literature review. Journal of Advanced Nursing, 53(2), 221-232.

Browne, M. W., \& Cudeck, R. (1993). Alternative ways of assessing model fit. Sage Focus Editions, 154, 136-162.

Burke, N. J., Hellman, J. L., Scott, B. G., Weems, C. F., \& Carrion, V. G. (2011). The impact of adverse childhood experiences on an urban pediatric population. Child Abuse \& Neglect, 35(6), 408-413.

Burnes, B. (2011). Introduction: Why does change fail, and what can we do about it? Journal of Change Management, 11(4), 445-450.

Burton, R. F. (2005). Multiple- choice and true/false tests: Myths and misapprehensions. Assessment \& Evaluation in Higher Education, 30(1), 65-72. 
Bussey, M. C. (2008). Trauma response and recovery certificate program: Preparing students for effective practice. Journal of Teaching in Social Work, 28(1-2), $117-144$.

Butler, L. D., \& Wolf, M. R. (2009). Trauma-informed care: Trauma as an organizing principle in the provision of mental health and social service. Trauma Psychology Newsletter, 4(3), 7-11.

Byrne, B. M. (2016). Structural equation modeling with AMOS: Basic concepts, applications, and programming. New York, NY: Routledge.

Byrne, S., Barry, D., \& Petry, N. M. (2012). Predictors of weight loss success: Exercise vs. dietary self-efficacy and treatment attendance. Appetite, 58(2), 695-698.

Caldwell, S. D. (2013). Are change readiness strategies overrated? A commentary on boundary conditions. Journal of Change Management, 13(1), 19-35.

Caldwell, S., Liu, Y., Fedor, D. B., \& Herold, D. M. (2009). Why are perceptions of change in the "eye of the beholder"? The role of age, sex, and tenure in procedural justice judgments. The Journal of Applied Behavioral Science, 45(3), 437-459.

Campbell, D. E. (2011). How to write good multiple- choice questions. Journal of Pediatrics and Child Health, 47(6), 322-325. 
Canrinus, E. T., Helms-Lorenz, M., Beijaard, D., Buitink, J., \& Hofman, A. (2012). Selfefficacy, job satisfaction, motivation and commitment: Exploring the relationships between indicators of teachers' professional identity. European Journal of Psychology of Education, 27(1), 115-132.

Case, S. M., \& Swanson, D. B. (2002). Constructing written test questions for the basic and clinical sciences (3rd ed.). Philadelphia, PA: National Board of Medical Examiners. Retrieved from http://www.nbme.org/PDF/ItemWriting_2003/2003IWGwhole.pdf.

Chen, J., \& Wang, L. (2007). Locus of control and the three components of commitment to change. Personality and Individual Differences, 42(3), 503-512.

Choi, M. (2011). Employees' attitudes toward organizational change: A literature review. Human Resource Management, 50(4), 479-500.

Choi, M., \& Ruona, W. E. (2011). Individual readiness for organizational change and its implications for human resource and organization development. Human Resource Development Review, 10(1), 46-73.

Clark, C., Young, M. S., Jackson, E., Graeber, C., Mazelis, R., Kammerer, N., \& Huntington, N. (2008). Consumer perceptions of integrated trauma-informed services among women with co-occurring disorders. The Journal of Behavioral Health Services \& Research, 35(1), 71-90. 
Coffman, J. (2007). A framework for evaluating systems initiatives. Build Initiative. Retrieved from http://pathways.nccp.org/assets/pdf/Coffman2007.pdf.

Cohen, K., \& Collens, P. (2013). The impact of trauma work on trauma workers: A metasynthesis on vicarious trauma and vicarious posttraumatic growth. Psychological Trauma: Theory, Research, Practice, and Policy, 5(6), 570.

Corbin, T. J., Purtle, J., Rich, L. J., Rich, J. A., Adams, E. J., Yee, G., \& Bloom, S. L. (2013). The prevalence of trauma and childhood adversity in an urban, hospitalbased violence intervention program. Journal of Health Care for the Poor and Underserved, 24(3), 1021-1030.

Council of Social Work Education. (2008). Educational policy and accreditation standards. Alexandria, VA: Author. Retrieved from https://www.cswe.org/Accreditation/Standards-and-Policies/2008-EPAS

Council on Social Work Education. (2012). Advanced social work practice in trauma. Alexandria, VA: Author. Retrieved from https://csbs.uni.edu/sites/default/files/allfiles/CSWE\%20\%20Trauma\%20Informed\%20Practice\%20Behaviors.pdf Council of Social Work Education. (2015). Educational policy and accreditation standards. Alexandria, VA: Author. Retrieved from https://www.cswe.org/getattachment/Accreditation/Accreditation-Process/2015EPAS/2015EPAS_Web_FINAL.pdf.aspx 
Courtois, C. A. (2002). Traumatic stress studies: The need for curricula inclusion. Journal of Trauma Practice, 1(1), 33-57.

Courtois, C. A., \& Gold, S. N. (2009). The need for inclusion of psychological trauma in the professional curriculum: A call to action. Psychological Trauma: Theory, Research, Practice, and Policy, 1(1), 3.

Damschroder, L. J., Aron, D. C., Keith, R. E., Kirsh, S. R., Alexander, J. A., \& Lowery, J. C. (2009). Fostering implementation of health services research findings into practice: A consolidated framework for advancing implementation science. Implement Science, 4(1), 50.

Damschroder, L. J., \& Hagedorn, H. J. (2011). A guiding framework and approach for implementation research in substance use disorders treatment. Psychology of Addictive Behaviors, 25(2), 194.

Davis, F. D., \& Venkatesh, V. (1996). A critical assessment of potential measurement biases in the technology acceptance model: three experiments. International Journal of Human-Computer Studies, 45(1), 19-45.

Decker, P., Durand, R., Mayfield, C. O., McCormack, C., Skinner, D., \& Perdue, G. (2012). Predicting implementation failure in organization change. Journal of Organizational Culture, Communication and Conflict, 16(2), 29. 
Devos, G., Buelens, M., \& Bouckenooghe, D. (2007). Contribution of content, context, and process to understanding openness to organizational change: Two experimental simulation studies. The Journal of Social Psychology, 147(6), 607630.

DiBattista, D., \& Kurzawa, L. (2011). Examination of the quality of multiple-choice items on classroom tests. Canadian Journal for the Scholarship of Teaching and Learning, 2(2), 4.

Dimeff, L. A., Koerner, K., Woodcock, E. A., Beadnell, B., Brown, M. Z., Skutch, J. M.,...Harned, M.S.. (2009). Which training method works best? A randomized controlled trial comparing three methods of training clinicians in dialectical behavior therapy skills. Behavioural Research and Therapy, 47, 921-930.

Dingfelder, H. E., \& Mandell, D. S. (2011). Bridging the research-to-practice gap in autism intervention: An application of diffusion of innovation theory. Journal of Autism and Developmental Disorders, 41(5), 597-609.

Dinnen, S., Kane, V., \& Cook, J. M. (2014). Trauma-informed care: A paradigm shift needed for services with homeless veterans. Professional Case Management, 19(4), 161-170.

Drzensky, F., Egold, N., \& van Dick, R. (2012). Ready for a change? A longitudinal study of antecedents, consequences and contingencies of readiness for change. Journal of Change Management, 12(1), 95-111. 
Dube, S. R., Felitti, V. J., Dong, M., Giles, W. H., \& Anda, R. F. (2003). The impact of adverse childhood experiences on health problems: Evidence from four birth cohorts dating back to 1900. Preventive Medicine, 37(3), 268-277.

Edwards, R. W., Jumper-Thurman, P., Plested, B. A., Oetting, E. R., \& Swanson, L. (2000). Community readiness: Research to practice. Journal of Community Psychology, 28(3), 291-307.

Elliott, D. E., Bjelajac, P., Fallot, R.D., Markoff, L.S., \& Reed, B.G. (2005). Traumainformed or trauma-denied: Principles and implementation of trauma-informed services for women. Journal of Community Psychology, 33(4), 461-477. doi:10.1002/jcop.20063

Esaki, N., Hopson, L., \& Middleton, J. (2014). Sanctuary model implementation from the perspective of indirect care staff. Families in Society: The Journal of Contemporary Social Services, 95(4), 261-268.

Fairburn, C. G., \& Cooper, Z. (2011). Therapist competence, therapy quality, and therapist training. Behaviour Research and Therapy, 49(6), 373-378.

Fallot, R. D., \& Harris, M. (2006). Trauma-informed services: A self-assessment and planning protocol (version 1.4). Washington, DC: Community Connections.

Fallot, R. D. \& Harris, M. (2009). Creating cultures of trauma-informed care (CCTIC): A self-assessment and planning protocol. Washington, DC: Community Connections. 
Farro, S. A., Clark, C., \& Hopkins-Eyles, C. (2011). Assessing trauma-informed care readiness in behavioral health: An organizational case study. Journal of Dual Diagnosis, 7(4), 228-241.

Fedor, D. B., Caldwell, S., \& Herold, D. M. (2006). The effects of organizational changes on employee commitment: A multilevel investigation. Personnel Psychology, $59(1), 1-29$.

Felitti, V. J., Anda, R. F., Nordenberg, D., Williamson, D. F., Spitz, A. M., Edwards, V., Koss, M.P., \& Marks, J. S. (1998). Relationship of childhood abuse and household dysfunction to many of the leading causes of death in adults: The Adverse Childhood Experiences (ACE) Study. American Journal of Preventive Medicine, 14(4), 245-258.

Field, A. (2009). Discovering statistics using SPSS. Thousand Oaks, CA: Sage.

Foster, R. D. (2010). Resistance, justice, and commitment to change. Human Resource Development Quarterly, 21(1), 3-39.

Foster-Fishman, P. G., Nowell, B., \& Yang, H. (2007). Putting the system back into systems change: A framework for understanding and changing organizational and community systems. American Journal of Community Psychology, 39(3-4), 197215.

Fowler Jr, F. J. (2013). Survey research methods. Thousand Oaks, CA: Sage. 
Fraser, J. G., Griffin, J. L., Barto, B. L., Lo, C., Wenz-Gross, M., Spinazzola, J., ... \& Bartlett, J. D. (2014). Implementation of a workforce initiative to build traumainformed child welfare practice and services: Findings from the Massachusetts Child Trauma Project. Children and Youth Services Review, 44, 233-242.

Fricchione, G. L., Borba, C. P., Alem, A., Shibre, T., Carney, J. R., \& Henderson, D. C. (2012). Capacity building in global mental health: Professional training. Harvard Review of Psychiatry, 20(1), 47-57.

Fukukura, J., Ferguson, M. J., \& Fujita, K. (2013). Psychological distance can improve decision making under information overload via gist memory. Journal of Experimental Psychology, 142(3), 658.

Gerdes, K. E., \& Segal, E. (2011). Importance of empathy for social work practice: Integrating new science. Social Work, 56(2), 141-148.

Gibbons, C., Dempster, M., \& Moutray, M. (2011). Stress, coping and satisfaction in nursing students. Journal of Advanced Nursing, 67(3), 621-632.

Gierl, M. J., \& Haladyna, T. M. (Eds.). (2012). Automatic item generation: Theory and practice. New York, NY: Routledge.

Glaser, M., Langer, T., \& Weber, M. (2013). True overconfidence in interval estimates: Evidence based on a new measure of miscalibration. Journal of Behavioral Decision Making, 26(5), 405-417. 
Glasman, L. R., \& Albarracín, D. (2006). Forming attitudes that predict future behavior: A meta-analysis of the attitude-behavior relation. Psychological Bulletin, 132(5), 778.

Glisson, C. (2007). Assessing and changing organizational culture and climate for effective services. Research on Social Work Practice, 17(6), 736-747.

Goldner, E. M., Jenkins, E. K., \& Fischer, B. (2014). A narrative review of recent developments in knowledge translation and implications for mental health care providers. The Canadian Journal of Psychiatry, 59(3), 160-169.

Goodhue, D. L., \& Loiacono, E. T. (2002, January). Randomizing survey question order vs. grouping questions by construct: An empirical test of the impact on apparent reliabilities and links to related constructs. In Proceedings of the 35th Annual Hawaii International Conference on System Sciences (pp. 3456-3465). IEEE Computer Society: Washington, D.C.

Greener, J. M., Joe, G. W., Simpson, D. D., Rowan-Szal, G. A., \& Lehman, W. E. (2007). Influence of organizational functioning on client engagement in treatment. Journal of Substance Abuse Treatment, 33(2), 139-147.

Greenhalgh, T., \& Wieringa, S. (2011). Is it time to drop the 'knowledge translation' metaphor? A critical literature review. Journal of the Royal Society of Medicine, 104(12), 501-509. 
Greenwald, R., Maguin, E., Smyth, N. J., Greenwald, H., Johnston, K. G., \& Weiss, R. L. (2008). Teaching trauma-related insight improves attitudes and behaviors toward challenging clients. Traumatology, 14(2), 1.

Guarino, K., Soares, P., Konnath, K., Clervil, R., \& Bassuk, E. (2009). Trauma-informed organizational toolkit. Rockville, MD: Center for Mental Health Services, Substance Abuse and Mental Health Services Administration, and the Daniels Fund, the National Child Traumatic Stress Network, and the W.K. Kellogg Foundation. Retrieved from www.homeless.samhsa.gov and Www.familyhomelessness.org.

Gunnar, M., \& Quevedo, K. (2007). The neurobiology of stress and development. Annual Review of Psychology, 58, 145-173.

Hahn, A., Judd, C. M., Hirsh, H. K., \& Blair, I. V. (2014). Awareness of implicit attitudes. Journal of Experimental Psychology, 143(3), 1369.

Haladyna, T. M., Downing, S. M., \& Rodriguez, M. C. (2002). A review of multiplechoice item-writing guidelines for classroom assessment. Applied Measurement in Education, 15(3), 309-333.

Haladyna, T. M., \& Rodriguez, M. C. (2013). Developing and validating test items. New York, NY: Routledge.

Hall, C. M. (2011). Policy learning and policy failure in sustainable tourism governance: From first-and second-order to third-order change? Journal of Sustainable Tourism, 19(4-5), 649-671. 
Hall, G. E., \& Hord, S. M. (2006). Implementing change: Patterns, principles, and potholes ( $2^{\text {nd }}$ ed.). Boston, MA: Allyn \& Bacon. Retrieved from http://www.mathedleadership.org/docs/coaching/PL-B-HALL.pdf

Harris, M., \& Fallot, R., Eds. (2001). Using trauma theory to design service systems. San Francisco, CA: Jossey-Bass.

Health Resources and Services Administration. (2001). Healthy People 2010. Washington DC: National Academies Press.

Henderson, G. M. (2002). Transformative learning as a condition for transformational change in organizations. Human Resource Development Review, 1(2), 186-214.

Hendricks, A., Conradi, L., \& Wilson, C. (2011). Creating trauma-informed child welfare systems using a community assessment process. Child Welfare, 90(6), 187. Retrieved from http://www.chadwickcenter.org/CTISP/images/TICWPracticeToolkit.pdf

Herold, D. M., Fedor, D. B., \& Caldwell, S. D. (2007). Beyond change management: A multi-level investigation of contextual and personal influences on employees' commitment to change. Journal of Applied Psychology, 92, 942-951.

Herold, D. M., Fedor, D. B., Caldwell, S., \& Liu, Y. (2008). The effects of transformational and change leadership on employees' commitment to a change: A multilevel study. Journal of Applied Psychology, 93(2), 346. 
Herschell, A. D., Kolko, D. J., Baumann, B. L., \& Davis, A. C. (2010). The role of therapist training in the implementation of psychosocial treatments: A review and critique with recommendations. Clinical Psychology Review, 30(4), 448-466.

Herscovitch, L., \& Meyer, J. P. (2002). Commitment to organizational change: Extension of a three-component model. Journal of Applied Psychology, 87(3), 474.

Hilliard, T. M., \& Boulton, M. L. (2012). Public health workforce research in review: A 25-year retrospective. American Journal of Preventive Medicine, 42(5), S17-S28.

Hodas, G. (2006). Responding to childhood trauma: The promise and practice of traumainformed care. Philadelphia, PA: Office of Mental Health and Substance Abuse Services. Retrieved from http://www.childrescuebill.org/VictimsOfAbuse/RespondingHodas.pdf

Hodgdon, H. B., Kinniburgh, K., Gabowitz, D., Blaustein, M. E., \& Spinazzola, J. (2013). Development and implementation of trauma-informed programming in youth residential treatment centers using the ARC framework. Journal of Family Violence, 28(7), 679-692.

Holt, D. T., Armenakis, A. A., Feild, H. S., \& Harris, S. G. (2007). Readiness for organizational change: The systematic development of a scale. The Journal of Applied Behavioral Science, 43(2), 232-255.

Holt, D. T., Helfrich, C. D., Hall, C. G., \& Weiner, B. J. (2010). Are you ready? How health professionals can comprehensively conceptualize readiness for change. Journal of General Internal Medicine, 25(1), 50-55. 
Holt, D. T., \& Vardaman, J. M. (2013). Toward a comprehensive understanding of readiness for change: The case for an expanded conceptualization. Journal of Change Management, 13(1), 9-18.

Hopper, E. K., Bassuk, E. L., \& Olivet, J. (2010). Shelter from the storm: Traumainformed care in homelessness services settings. Open Health Services and Policy Journal, 2, 131-151.

Howard, G. S., \& Dailey, P. R. (1979). Response-shift bias: A source of contamination of self-report measures. Journal of Applied Psychology, 64(2), 144-150.

Institute for Health and Recovery. (2014). Developing trauma informed organizations: A tool kit. Cambridge, MA. http://www.healthrecovery.org/publications/detail.php?p=30)

Jack-Walker, H., Armenakis, A. A., \& Bernerth, J. B. (2007). Factors influencing organizational change efforts: An integrative investigation of change content, context, process and individual differences. Journal of Organizational Change Management, 20(6), 761-773.

Jamieson, D., \& Gellermann, W. (2014). Values, ethics, and OD practice. The NTL Handbook of Organization Development and Change, 45-65.

Jaros, S. (2010). Commitment to organizational change: A critical review. Journal of Change Management, 10(1), 79-108. 
Jennings, A. (2004). Models for developing trauma-informed behavioral health systems and trauma-specific services. Alexandria, VA: National Association of State Mental Health Program Directors, National Technical Assistance Center for State Mental Health Planning.

Jones, R. A., Jimmieson, N. L., \& Griffiths, A. (2005). The impact of organizational culture and reshaping capabilities on change implementation success: The mediating role of readiness for change. Journal of Management Studies, 42(2), 361-386.

Kazemi, D. M., Wagenfeld, M., Van Horn, K. R., Levine, M. J., \& Dmochowski, J. (2011). Binge drinking among underage college students: Role of impulsivity and the transtheoretical model. Journal of Addictions Nursing, 22(4), 193-199.

Keesler, J. M. (2014). A call for the integration of trauma-informed care among intellectual and developmental disability organizations. Journal of Policy and Practice in Intellectual Disabilities, 11(1), 34-42.

Kenny, D. A. (2015). Measuring model fit. Retrieved http://davidakenny.net/cm/fit.htm Kenny, D.A. (2016) Mediation. Retrieved from http://davidakenny.net/cm/mediate.htm

Kerby, D. S., Brand, M. W., Elledge, B. L., Johnson, D. L., \& Magas, O. K. (2005). Are public health workers aware of what they don't know? Biosecurity and Bioterrorism: Biodefense Strategy, Practice, and Science, 3(1), 31-38. 
Kim, T. G., Hornung, S., \& Rousseau, D. M. (2011). Change-supportive employee behavior: Antecedents and the moderating role of time. Journal of Management, 37(6), 1664-1693.

Kinman, G., \& Grant, L. (2011). Exploring stress resilience in trainee social workers: The role of emotional and social competencies. British Journal of Social Work, 4l(2), 261-275.

Klarner, P., \& Raisch, S. (2013). Move to the beat: Rhythms of change and firm performance. Academy of Management Journal, 56(1), 160-184.

Kline, R. B. (2011). Principles and practice of structural equation modeling ( $3^{\text {rd }}$ ed.). New York, NY: Guilford.

Ko, S. J., Ford, J. D., Kassam-Adams, N., Berkowitz, S. J., Wilson, C., Wong, M., Brymer, M.J., \& Layne, C. M. (2008). Creating trauma-informed systems: Child welfare, education, first responders, health care, juvenile justice. Professional Psychology: Research and Practice, 39(4), 396-404. doi: http://dx.doi.org/10.1037/0735-7028.39.4.396

Koh, H. K., Oppenheimer, S. C., Massin-Short, S. B., Emmons, K. M., Geller, A. C., \& Viswanath, K. (2010). Translating research evidence into practice to reduce health disparities: A social determinants approach. American Journal of Public Health, 100(S1), S72-S80. 
Kramer, T. L., Sigel, B. A., Conners-Burrow, N. A., Savary, P. E., \& Tempel, A. (2013). A statewide introduction of trauma-informed care in a child welfare system. Children and Youth Services Review, 35(1), 19-24.

Krathwohl, D. R. (2002). A revision of Bloom's taxonomy: An overview. Theory into Practice, 41(4), 212-218.

Kuipers, B. S., Higgs, M., Kickert, W., Tummers, L., Grandia, J., \& Van der Voet, J. (2014). The management of change in public organizations: A literature review. Public Administration, 92(1), 1-20.

Kulkarni, S., Bell, H., Hartman, J., L., \& Herman-Smith, R.L. (2013). Exploring individual and organizational factors contributing to compassion satisfactions, secondary traumatic stress, and burnout in domestic violence service providers. Journal of the Society for Social Work and Research, 4(2), 114-130.

Kusmaul, N., Wilson, B., \& Nochajski, T. (2015). The infusion of trauma-informed carein organizations: Experience of agency staff. Human Service Organizations: Management, Leadership \& Governance, 39(1). doi: $10.1080 / 23303131.2014 .968749$

Kwahk, K. Y., \& Kim, H. W. (2008). Managing readiness in enterprise systems-driven organizational change. Behaviour \& Information Technology, 27(1), 79-87.

Ladwig, P., Dalrymple, K. E., Brossard, D., Scheufele, D. A., \& Corley, E. A. (2012). Perceived familiarity or factual knowledge? Comparing operationalizations of scientific understanding. Science and Public Policy, 39(6), 761-774. 
Langley, A., Smallman, C., Tsoukas, H., \& Van de Ven, A. H. (2013). Process studies of change in organization and management: Unveiling temporality, activity, and flow. Academy of Management Journal, 56(1), 1-13.

Layne, C. M., Ippen, C. G., Strand, V., Stuber, M., Abramovitz, R., Reyes, G., ... \& Pynoos, R. (2011). The core curriculum on childhood trauma: A tool for training a trauma-informed workforce. Psychological Trauma: Theory, Research, Practice, and Policy, 3(3), 243.

LeBel, J., \& Kelly, N. (2014). 7 Trauma-informed care. Residential Interventions for Children, Adolescents, and Families: A Best Practice Guide, 78.

Lehman, W. E. K., Greener, J. M., \& Simpson, D. D. (2002). Assessing organizational readiness for change. Journal of Substance Abuse Treatment, 22(4), 197-209.

Lehman, W. E., Greener, J. M., Rowan-Szal, G. A., \& Flynn, P. M. (2012). Organizational readiness for change in correctional and community substance abuse programs. Journal of Offender Rehabilitation, 51(1-2), 96-114.

Lehman, W. E., Simpson, D. D., Knight, D. K., \& Flynn, P. M. (2011). Integration of treatment innovation planning and implementation: strategic process models and organizational challenges. Psychology of Addictive Behaviors, 25(2), 252.

Lewin, K. (1951). Field theory in social science. New York, NY: Harper. 
Liu, Y., Caldwell, S. D., Fedor, D. B., \& Herold, D. M. (2012). When does management's support for a change translate to perceptions of fair treatment? The moderating roles of change attributions and conscientiousness. The Journal of Applied Behavioral Science, 48(4), 441-462.

Lloyd, F. J., \& Reyna, V. F. (2009). Clinical gist and medical education: Connecting the dots. JAMA, 302(12), 1332-1333.

Lowe, S. R., Blachman-Forshay, J., \& Koenen, K. C. (2015). Trauma as a public health issue: Epidemiology of trauma and trauma-related disorders. In U. Schnyder \& M. Cloitre (Eds.), Evidence Based Treatments for Trauma-Related Psychological Disorders (pp. 11-40). doi: 10.1007/978-3-319-07109-1_2.

Luiselli, J. K., Bass, J. D., \& Whitcomb, S. A. (2010). Teaching applied behavior analysis knowledge competencies to direct-care service providers: Outcome assessment and social validation of a training program. Behavior Modification, 34(5), 403414.

MacKay, R. B., \& Chia, R. (2013). Choice, chance, and unintended consequences in strategic change: A process understanding of the rise and fall of Northco Automotive. Academy of Management Journal, 56(1), 208-230.

Mackay, T. L., Petermann, L., Hurrell, C., \& Hodgins, D. (2015). Knowledge translation in gambling research: A scoping review. International Gambling Studies, 15(2), 179-195. 
MacKinnon, D. P. (2008). Introduction to statistical mediation analysis. New York, NY: Routledge.

Maes, G., \& Van Hootegem, G. (2011). Toward a dynamic description of the attributes of organizational change. In A.B. Shani, R.W. Woodman, \& W.A. Pasmore (Eds.), Research in Organizational Change and Development (pp. 191-231). Bingley, UK: Emerald Group Publishing Limited. doi:10.1108/S08973016(2011)0000019009

Markoff, L. S., Finkelstein, N., Kammere, N., Kreiner, P., \& Prost, C. A. (2005). Relational systems change. The Journal of Behavioral Health Services \& Research, 32(2), 227-240.

Marlowe, J., \& Adamson, C. (2011). Teaching trauma: Critically engaging a troublesome term. Social Work Education, 30(6), 623-634.

Martino, S., Ball, S. A., Nich, C., Canning-Ball, M., Rounsaville, B. J., \& Carroll, K. M. (2011). Teaching community program clinicians motivational interviewing using expert and train-the-trainer strategies. Addiction, 106(2), 428-441.

McAllister, D., \& Guidice, R. M. (2012). This is only a test: A machine-graded improvement to the multiple-choice and true-false examination. Teaching in Higher Education, 17(2), 193-207.

McCoach, D. B., Gable, R. K., \& Madura, J. P. (2013). Instrument development in the affective domain: School and corporate applications ( ${ }^{\text {rd }}$ ed.). New York, NY: Springer. 
McEwen, B. S. (2008). Central effects of stress hormones in health and disease: Understanding the protective and damaging effects of stress and stress mediators. European Journal of Pharmacology, 583(2), 174-185.

McKenzie-Mohr, S. (2004). Creating space for radical trauma theory in generalist social work education. Journal of Progressive Human Services, 15(2), 45-55.

McKinsey \& Company. (2008, July). Creating organizational transformations. The McKinsey Quarterly, 1-7. Retrieved from http://www.mckinseyquarterly.com (accessed 9 January 2015).

Mersky, J. P., Topitzes, J., \& Reynolds, A. J. (2013). Impacts of adverse childhood experiences on health, mental health, and substance use in early adulthood: A cohort study of an urban, minority sample in the US. Child Abuse \& Neglect, 37(11), 917-925.

Meyer, J. P., Srinivas, E. S., Lal, J. B., \& Topolnytsky, L. (2007). Employee commitment and support for an organizational change: Test of the three-component model in two cultures. Journal of Occupational and Organizational Psychology, 80(2), $185-211$.

Meyer, J. P., Stanley, L. J., \& Vandenberg, R. J. (2013). A person-centered approach to the study of commitment. Human Resource Management Review, 23(2), 190-202.

Meyers, D. C., Durlak, J. A., \& Wandersman, A. (2012). The quality implementation framework: A synthesis of critical steps in the implementation process. American Journal of Community Psychology, 50(3-4), 462-480. 
Michalopoulos, L. M., \& Aparicio, E. (2012). Vicarious trauma in social workers: The role of trauma history, social support, and years of experience. Journal of Aggression, Maltreatment \& Trauma, 21(6), 646-664.

Michel, A., Stegmaier, R., \& Sonntag, K. (2010). I scratch your back-you scratch mine: Do procedural justice and organizational identification matter for employees' cooperation during change? Journal of Change Management, 10(1), 41-59.

Miller, N. A., \& Najavits, L. M. (2012). Creating trauma-informed correctional care: A balance of goals and environment. European Journal of Psychotraumatology, 3.

Mollart, L., Skinner, V. M., Newing, C., \& Foureur, M. (2013). Factors that may influence midwives' work-related stress and burnout. Women and Birth, 26(1), 26-32.

Momsen, J. L., Long, T. M., Wyse, S. A., \& Ebert-May, D. (2010). Just the facts? Introductory undergraduate biology courses focus on low-level cognitive skills. CBE-Life Sciences Education, 9(4), 435-440.

Morris, Z. S., Wooding, S., \& Grant, J. (2011). The answer is 17 years. What is the question: Understanding time lags in translational research. Journal of the Royal Society of Medicine, 104(12), 510-520.

Morrissey, J. P., Jackson, E. W., Ellis, A. R., Amaro, H., Brown, V. B., \& Najavits, L. M. (2005). Twelve-month outcomes of trauma-informed interventions for women with co-occurring disorders. Psychiatric Services, 56(10), 1213-1222. 
Moyers, T. B., Manuel, J. K., Wilson, P. G., Hendrickson, S. M. L., Talcott, W., \& Durand, P. (2008). A randomized trial investigating training in motivational interviewing for behavioral health providers. Behavioural and Cognitive Psychotherapy, 36, 149-162.

Muskett, C. (2014). Trauma-informed care in inpatient mental health settings: A review of the literature. International Journal of Mental Health Nursing, 23(1), 51-59.

Najavits, L. M., \& Hien, D. (2013). Helping vulnerable populations: A comprehensive review of the treatment outcome literature on substance use disorder and PTSD. Journal of Clinical Psychology, 69(5), 433-479.

National Association of Social Workers. (1999). Code of ethics of the National Association of Social Workers. Washington, DC: NASW Press.

National Association of State Mental Health Program Directors. (1998). Position statement on services and supports to trauma survivors. Retrieved from https://www.theannainstitute.org/NASMHPD_Position.pdf

Neff, K. D. (2011). Self-compassion, self-esteem, and well-being. Social and Personality Psychology Compass, 5(1), 1-12.

Neves, P. (2009). Readiness for change: Contributions for employees' level of individual change and turnover intentions. Journal of Change Management, 9(2), 215-231.

Neves, P., \& Caetano, A. (2009). Commitment to change: Contributions to trust in the supervisor and work outcomes. Group \& Organization Management, 34(6), 623644. 
Newell, J. M., \& MacNeil, G. A. (2010). Professional burnout, vicarious trauma, secondary traumatic stress, and compassion fatigue. Best Practices in Mental Health, 6(2), 57-68.

Nimon, K., Zigarmi, D., \& Allen, J. (2011). Measures of program effectiveness based on retrospective pretest data: Are all created equal? American Journal of Evaluation, 32(1), 8-28.

Nordin, N. (2012). The influence of leadership behavior and organizational commitment on organizational readiness for change in a higher learning institution. Asia Pacific Education Review, 13(2), 239-249.

Oordt, M. S., Jobes, D. A., Fonseca, V. P., \& Schmidt, S. M. (2009). Training mental health professionals to assess and manage suicidal behavior: Can provider confidence and practice behaviors be altered? Suicide and Life-Threatening Behavior, 39(1), 21-32.

Oreg, S. (2003). Resistance to change: Developing an individual differences measure. Journal of Applied Psychology, 88(4), 680.

Oreg, S. (2006). Personality, context, and resistance to organizational change. European Journal of Work and Organizational Psychology, 15(1), 73-101.

Oreg, S., Vakola, M., \& Armenakis, A. (2011). Change recipients' reactions to organizational change: A 60-year review of quantitative studies. The Journal of Applied Behavioral Science, 47(4), 461-524. 
Oregon Health Authority. (2015). Trauma informed services policy. Retrieved from http://www.oregon.gov/oha/amh/trauma-policy/Trauma\%20Policy.pdf

Pallant, J. (2010). SPSS survival manual (4 ${ }^{\text {th }}$ ed.). Berkshire, UK: McGraw-Hill Education.

Paterson, M., Lagosky, S., \& Mason, R. (2016). Health promotion and knowledge translation: Two roads to the same destination? Global Health Promotion, 0(0), 15. doi: $10.1177 / 1757975916665340$.

Phokeo, V., Sproule, B., \& Raman-Wilms, L. (2004). Community pharmacists' attitudes toward and professional interactions with users of psychiatric medication. Psychiatric Services, 55(12), 1434-1436.

Podsakoff, P. M., MacKenzie, S. B., \& Podsakoff, N. P. (2012). Sources of method bias in social science research and recommendations on how to control it. Annual Review of Psychology, 63, 539-569.

Polites, G. L., \& Karahanna, E. (2012). Shackled to the status quo: The inhibiting effects of incumbent system habit, switching costs, and inertia on new system acceptance. MIS Quarterly, 36(1), 21-42.

Pratt, C. C., McGuigan, W. M., \& Katzev, A. R. (2000). Measuring program outcomes: Using retrospective pretest methodology. American Journal of Evaluation, 21(3), 341-349.

Prochaska, J. O. (2013). Transtheoretical model of behavior change. In M. D. Gellman \& J. R. Turner (Eds.), Encyclopedia of behavioral medicine (pp. 1997-2000). New York, NY: Springer. 
Prochaska, J. O., \& DiClemente, C. C. (1982). Transtheoretical therapy: Toward a more integrative model of change. Psychotherapy: Theory, Research and Practice, 19, 276-288.

Rafferty, A. E., \& Griffin, M. A. (2006). Perceptions of organizational change: A stress and coping perspective. Journal of Applied Psychology, 91(5), 1154.

Rafferty, A. E., Jimmieson, N. L., \& Armenakis, A. A. (2013). Change readiness: A multilevel review. Journal of Management, 39(1), 110-135.

Rafferty, A. E., \& Simons, R. H. (2006). An examination of the antecedents of readiness for fine-tuning and corporate transformation changes. Journal of Business and Psychology, 20(3), 325-350.

Reeves, E. (2015). A synthesis of the literature on trauma-informed care. Issues in Mental Health Nursing, 36(9), 698-709.

Reyna, V. F. (2008). A theory of medical decision making and health: Fuzzy trace theory. Medical Decision Making, 28(6), 850-865.

Reyna, V. F. (2012). A new intuitionism: Meaning, memory, and development in fuzzytrace theory. Judgment and Decision Making, 7(3), 332.

Richardson, M. M., Coryn, C. L., Henry, J., Black-Pond, C., \& Unrau, Y. (2012). Development and evaluation of the trauma-informed system change instrument: Factorial validity and implications for use. Child and Adolescent Social Work Journal, 29(3), 167-184. 
Roediger, H. L., \& Butler, A. C. (2011). The critical role of retrieval practice in longterm retention. Trends in Cognitive Sciences, 15(1), 20-27.

Rogers, E. M. (2003). The diffusion of innovations (5th ed.). New York, NY: Free Press. Sandberg, J., \& Tsoukas, H. (2011). Grasping the logic of practice: Theorizing through practical rationality. Academy of Management Review, 36(2), 338-360.

Santhidran, S., Chandran, V. G. R., \& Borromeo, J. (2013). Enabling organizational change-leadership, commitment to change and the mediating role of change readiness. Journal of Business Economics and Management, 14(2), 348-363.

Satorra, A., \& Bentler, P.M. (1988). Scaling corrections for chi-square statistics in covariance structure analysis. American Statistical Association 1988 proceedings of the Business and Economics section (pp. 308-313). Alexandria, VA: American Statistical Association.

Savage, A., Quiros, L., Dodd, S. J., \& Bonavota, D. (2007). Building trauma informed practice: Appreciating the impact of trauma in the lives of women with substance abuse and mental health problems. Journal of Social Work Practice in the Addictions, 7(1-2), 91-116.

Schwartz, C., \& Sprangers, M. (2010). Guidelines for improving the stringency of response shift research using the Thentest. Quality Of Life Research, 19, 455-464.

Sedikides, C., \& Gregg, A. P. (2008). Self-enhancement: Food for thought. Perspectives on Psychological Science, 3(2), 102-116. 
Self, D. R., \& Schraeder, M. (2009). Enhancing the success of organizational change: Matching readiness strategies with sources of resistance. Leadership \& Organization Development Journal, 30(2), 167-182.

Shin, J., Taylor, M. S., \& Seo, M. G. (2012). Resources for change: The relationships of organizational inducements and psychological resilience to employees' attitudes and behaviors toward organizational change. Academy of Management Journal, 55(3), 727-748.

Sholomskas, D. E., Syracuse-Siewert, G., Rounsaville, B. J., Ball, S. A., Nuro, K. F., \& Carroll, K. M. (2005). We don't train in vain: A dissemination trial of three strategies for training clinicians in cognitive-behavioral therapy. Journal of Consulting and Clinical Psychology, 73(1), 106.

Shonkoff, J. P., Garner, A. S., Siegel, B. S., Dobbins, M. I., Earls, M. F., McGuinn, L., ... \& Wood, D. L. (2012). The lifelong effects of early childhood adversity and toxic stress. Pediatrics, 129(1), e232-e246.

Simpson, D. D. (2002). A conceptual framework for transferring research to practice. Journal of Substance Abuse Treatment, 22(4), 171-182.

Singleton Jr, R. A., Straits, B. C., \& Straits, M. M. (2010). Approaches to social research ( $5^{\text {th }}$ ed.). New York, NY: Oxford University Press.

Sitzmann, T., Ely, K., Brown, K. G., \& Bauer, K. N. (2010). Self-assessment of knowledge: A cognitive learning or affective measure? Academy of Management Learning \& Education, 9(2), 169-191. 
Sobel, M.E. (1986). Some new results on indirect effects and their standard errors in covariance structure models. In N.B. Tuma (Ed.), Sociological methodology (pp. 159-186). San Francisco, CA: Jossey-Bass.

Solomons, N. M., \& Spross, J. A. (2011). Evidence-based practice barriers and facilitators from a continuous quality improvement perspective: An integrative review. Journal of Nursing Management, 19(1), 109-120.

Sprang, G., Craig, C., \& Clark, J. (2011). Secondary traumatic stress and burnout in child welfare workers: A comparative analysis of occupational distress across professional groups. Child Welfare, 90(6), 149.

Stirman, S. W., Gutiérrez-Colina, A., Toder, K., Esposito, G., Barg, F., Castro, F., ... \& Crits-Christoph, P. (2013). Clinicians' perspectives on cognitive therapy in community mental health settings: Implications for training and implementation. Administration and Policy in Mental Health and Mental Health Services Research, 40(4), 274-285.

Strand, V. C., Abramovitz, R., Layne, C. M., Robinson, H., \& Way, I. (2014). Meeting the critical need for trauma education in social work: A problem-based learning approach. Journal of Social Work Education, 50(1), 120-135.

Substance Abuse and Mental Health Services Administration. (2012). SAMHSA's working definition of trauma and principles and guidance for a traumainformed approach [Draft]. Rockville, MD: Author. 
Substance Abuse and Mental Health Services Administration. (2014a). Trauma informed care in behavioral health services. Treatment Improvement Protocol (TIP) Series 57. HHS Publication No. (SMA) 13-4801. Rockville, MD: Author. Substance Abuse and Mental Health Services Administration. (2014b). SAMHSA'S concept of trauma and guidance for a trauma informed approach. HHS Publication No. (SMA) xx-xxxx. Rockville, MD: Author.

Substance Abuse and Mental Health Administration. (2015). Trauma informed approach and trauma specific interventions. Retrieved from http://www.samhsa.gov/nctic/trauma-interventions

Tarrant, M., \& Ware, J. (2012). A framework for improving the quality of multiplechoice assessments. Nurse Educator, 37(3), 98-104.

Tasdemir, M. (2010). A comparison of multiple-choice tests and true-false tests used in evaluating student progress. Journal of Instructional Psychology, 37(3), 258-267.

Taylor, A., Cocklin, C., \& Brown, R. (2012). Fostering environmental champions: A process to build their capacity to drive change. Journal of Environmental Management, 98, 84-97.

Trauma Informed Oregon. (2016). Prioritize and create a work plan. Retrieved from http://traumainformedoregon.org/wp-content/uploads/2016/05/Prioritize-andCreate-Work-Plan-Considerations-Page.pdf

Tsoukas, H., \& Chia, R. (2002). On organizational becoming: Rethinking organizational change. Organization Science, 13(5), 567-582. 
United States Census Bureau. (n.d.). 2011-2015 American community survey 5-year estimates. Retrieved from https://factfinder.census.gov/faces/tableservices/jsf/pages/productview.xhtml?pid =ACS_15_5YR_B02001\&prodType=table

Vakola, M. (2014). What's in there for me? Individual readiness to change and the perceived impact of organizational change. Leadership \& Organization Development Journal, 35(3), 195-209.

Vakola, M., \& Nikolaou, I. (2005). Attitudes towards organizational change: What is the role of employees' stress and commitment? Employee Relations, 27(2), 160-174.

VanDeusen-Lukas, C. V., Meterko, M. M., Mohr, D., Seibert, M. N., Parlier, R., Levesque, O., \& Petzel, R. A. (2008). Implementation of a clinical innovation: The case of advanced clinic access in the Department of Veterans Affairs. The Journal of Ambulatory Care Management, 31(2), 94-108.

Van Niel, C., Pachter, L. M., Wade Jr, R., Felitti, V. J., \& Stein, M. T. (2014). Adverse events in children: Predictors of adult physical and mental conditions. Journal of Developmental \& Behavioral Pediatrics, 35(8), 549-551.

Vijayalakshmi, V., \& Bhattacharyya, S. (2012). Emotional contagion and its relevance to individual behavior and organizational processes: A position paper. Journal of Business and Psychology, 27(3), 363-374.

Wanberg, C. R., \& Banas, J. T. (2000). Predictors and outcomes of openness to changes in a reorganizing workplace. Journal of Applied Psychology, 85(1), 132. 
Weiner, B., Amick, H., \& Lee, S. Y. (2008). Conceptualization and measurement of organizational readiness for change: A review of the literature in health services research and other fields. Medical Care Research and Review, 65(4), 379-436.

Williams, D. R., Costa, M. V., Odunlami, A. O., \& Mohammed, S. A. (2008). Moving upstream: How interventions that address the social determinants of health can improve health and reduce disparities. Journal of Public Health Management and Practice, 14(S), S8-17. http://doi.org.proxy.lib.pdx.edu/10.1097/01.PHH.0000338382.36695.42

Wilson, E. V., \& Lankton, N. K. (2012, December). Some unfortunate consequences of nonrandomized, grouped-item survey administration in IS research. In Proceedings of the 2012 International Conference on Information Systems (ICIS), Orlando, FL. Retrieved from https://works-bepresscom.proxy.lib.pdx.edu/nancy_lankton/9/

Wisdom, J. P., Chor, K. H. B., Hoagwood, K. E., \& Horwitz, S. M. (2014). Innovation adoption: A review of theories and constructs. Administration and Policy in Mental Health and Mental Health Services Research, 41(4), 480-502.

Wolf, M. R., Green, S. A., Nochajski, T. H., Mendel, W. E., \& Kusmaul, N. S. (2014). 'We're civil servants': The status of trauma-informed care in the community. Journal of Social Service Research, 40(1), 111-120. 
World Health Organization. (2014). Closing the gap in a generation: Health equity through action on the social determinants of health. Commission on the Social Determinants of Health. Geneva, Switzerland: Author.

Yatchmenoff, D. K., Sundborg, S. A., \& Davis, M. A. (in press). Implementing Trauma Informed Care in Organizations: Observations and Recommendations on the Process. Advances in Social Work. 


\section{Appendix A}

\section{Sample Demographics}

Table A1.

Amount of Prior Training across Multiple Formats

\begin{tabular}{lllll}
\hline Training Method & A lot & Some & Very little & None \\
\hline Training: In person & $4(3.4 \%)$ & $34(28.8 \%)$ & $52(44.1 \%)$ & $17(14.4 \%)$ \\
Training: Webinars & $28(23.7 \%)$ & $39(33.1 \%)$ & $35(29.7 \%)$ & $5(4.2 \%)$ \\
Training: Books & $15(12.7 \%)$ & $26(22.0 \%)$ & $44(37.3 \%)$ & $21(17.8 \%)$
\end{tabular}

Note . Total sample $=118$; Training in Person, $\mathrm{N}=107$; Training through Webinars, $\mathrm{N}=107$; Training Through Books, $\mathrm{N}=106$.

Table A2.

Length of Time Since Training

\begin{tabular}{lcccc}
\hline "How long has it been & $<1$ month & $\begin{array}{c}2-6 \\
\text { months }\end{array}$ & $\begin{array}{l}7-12 \\
\text { months }\end{array}$ & $\begin{array}{l}\text { More than } \\
\text { one year }\end{array}$ \\
\cline { 2 - 5 } $\begin{array}{l}\text { since your last training } \\
\text { on the topics of trauma } \\
\text { and trauma-informed }\end{array}$ & 34 & 31 & 26 & 15 \\
care?" & $(28.8 \%)$ & $(26.3 \%)$ & $(22.0 \%)$ & $(12.7 \%)$ \\
\hline $\begin{array}{l}\text { Note. Total respondents on this question, n=106. } \\
\end{array}$ & & & \\
\end{tabular}


Table A3.

Occupational Field and Role

\begin{tabular}{lr}
\hline Field & $\mathrm{N}(\%)$ \\
\hline Physical health & $1(0.8 \%)$ \\
Mental health & $49(41.5 \%)$ \\
Public health & $35(29.7 \%)$ \\
Substance abuse services & $1(0.8 \%)$ \\
Criminal justice/law enforcement & $1(0.8 \%)$ \\
Education & $2(1.7 \%)$ \\
Early childhood & $10(8.5 \%)$ \\
Other & $8(6.8 \%)$ \\
\hline Role & \\
\hline Direct service & $60(50.8 \%)$ \\
Administrative or support & $14(11.9 \%)$ \\
Management & $22(18.6 \%)$ \\
Other & $11(9.3 \%)$ \\
\hline
\end{tabular}

Note. Total sample $=118$; Responses about field, $\mathrm{n}=107$; Responses about role, $\mathrm{n}=107$.

${ }^{\mathrm{a}}$ Management $=$ directors, managers, and supervisors.

Table A4.

Years of Experience

\begin{tabular}{lllll}
\hline Experience & $<2$ yrs & $2-5$ yrs & $6-10$ yrs & $\begin{array}{l}\text { More than } \\
10 \text { yrs }\end{array}$ \\
\hline Yrs at current organization & $\begin{array}{l}21 \\
(17.8 \%)\end{array}$ & $\begin{array}{c}44 \\
(37.3 \%)\end{array}$ & $\begin{array}{c}19 \\
(16.1 \%)\end{array}$ & $\begin{array}{c}23 \\
(19.5 \%)\end{array}$ \\
& & & & \\
Yrs in current field & 4 & 32 & 29 & 42 \\
& $(3.4 \%)$ & $(27.1 \%)$ & $(24.6 \%)$ & $(35.6 \%)$ \\
& & & & 82 \\
Yrs in workforce (any & 1 & 4 & 20 & $(69.5 \%)$ \\
field) & $(0.8 \%)$ & $(3.4 \%)$ & $(16.9 \%)$ & \\
\hline Note. Total sample = 118; Response about years of experience, $\mathrm{n}=107$. &
\end{tabular}




\section{Appendix B}

\section{Measures Included in the Study}

Filtering Question. Answer choices 1=strongly disagree, 2=moderately disagree, $3=$ mildly disagree, $4=$ not sure, $5=$ mildly agree, $6=$ moderately agree $7=$ strongly agree .

1. My organization is currently working to implement Trauma-Informed Care. Affective Commitment to Trauma-Informed Care. Answer choices 1=strongly disagree, $2=$ moderately disagree, $3=$ mildly disagree, $4=$ not sure, $5=$ mildly agree, 6=moderately agree $7=$ strongly agree.

1. I believe in the value of Trauma-Informed Care.

2. Trauma-Informed Care is a good strategy for this organization.

3. I think that management is [or would be] making a mistake by introducing TraumaInformed Care. $\mathrm{R}$

4. Trauma-Informed Care serves an important purpose.

5. Things would be better without Trauma-Informed Care. $\mathrm{R}$

6. Trauma-Informed Care is not necessary. $\mathrm{R}$

Foundational Knowledge About Trauma-Informed Care. The following statement introduced the knowledge section of the survey, "The following questions ask about your knowledge of trauma, the impact of trauma, and Trauma-Informed Care (TIC). Please select the answer that most closely represents your knowledge." Answer choices $1=$ completely untrue, $2=$ somewhat untrue, $3=$ somewhat true $4=$ completely true. 
Subheadings did not appear in Qualtrics.

\section{Trauma in the workforce}

1. I understand the signs and symptoms of work related stress including secondary traumatic stress, vicarious trauma, compassion fatigue, and burnout.

2. I can tell the difference between secondary traumatic stress, vicarious trauma, and burnout.

3. I can explain to others, the difference between secondary traumatic stress, vicarious trauma, and burnout.

4. I know the importance of self-care for the workforce.

\section{Principles and strategies of Trauma-Informed Care}

5. I know the principles of Trauma-Informed Care.

6. I can explain to others, the principles of Trauma-Informed Care.

7. I know how to review policy, practice, and procedures using a trauma lens.

8. I can identify strategies to be more trauma informed in my agency.

9. I understand the difference between trauma specific services and TraumaInformed Care.

\section{Neurobiology of stress and trauma}

10. I understand the reasons why individuals respond to trauma differently.

11. I can explain to others, the reasons why individuals respond to trauma differently.

12. I understand that a stress response can be activated in the absence of real threat. 
13. I understand how trauma can affect cognitive process such as memory, attention, and perception.

14. I can explain to others, how trauma can affect cognitive process such as memory, attention, and perception.

15. I understand how trauma can affect relationships and attachment.

16. I understand how trauma can affect emotional regulation.

17. I know the signs of an acute stress response.

18. I know what is happening in the mind and body during an acute stress response.

19. I understand why unresolved trauma exposure has a cumulative impact over time on individual, family, organizational, and community functioning.

\section{Adverse Childhood Experiences (ACE) Study}

20. I know about the Adverse Childhood Experiences (ACE) study conducted by Kaiser Permanente and the CDC.

21. I know which traumas were included in the ACE study.

22. I understand the dose-response relationship between adverse experiences and negative outcomes.

23. I can explain to others, the findings from the ACE study.

24. I am familiar with the ACE pyramid and how adverse childhood experiences influence health and well-being.

\section{Historical oppression and intergenerational trauma}

25. I understand how trauma can be transferred from one generation to the next. 
26. I know one method of transferring trauma from one generation to the next is biologically through altered DNA.

\section{Issues of power and oppression related to the experience of trauma}

27. I understand how economic inequities adversely affect experiences of trauma and access to resources that facilitate resilience and recovery.

28. I understand how race, class, gender, sexual orientation, religion, and national origin can result in disproportionate trauma exposure.

29. I understand how vulnerable and marginalized people and their communities can be differentially impacted by trauma.

30. I understand how historical and structural oppression can create traumatic conditions.

Principal Support for Trauma-Informed Care. Answer choices 1=strongly disagree, $2=$ moderately disagree, $3=$ mildly disagree, $4=$ not sure, $5=$ mildly agree, $6=$ moderately agree $7=$ strongly agree.

1. Most of my respected peers have embraced Trauma-Informed Care.

2. The top leaders in this organization are "walking the talk".

3. The top leaders support Trauma-Informed Care.

4. The majority of my respected peers are dedicated to making Trauma-Informed Care successful.

5. My immediate manager encourages me to support Trauma-Informed Care. 6. My immediate manager is in favor of Trauma-Informed Care. 
TIC Self-Efficacy. Answer choices 1=strongly disagree, $2=$ =moderately disagree, $3=$ mildly disagree, $4=$ not sure, $5=$ mildly agree, $6=$ moderately agree $7=$ strongly agree .

1. I do not anticipate any problems adjusting to the work I will have when TraumaInformed Care is adopted.

2. There are tasks that will be required with Trauma-Informed Care that I don't think I can do well.

3. When we implement Trauma-Informed Care, I feel I can handle it with ease.

4. I have the skills that are needed to make Trauma-Informed Care work.

5. I have the knowledge that is needed to make Trauma-Informed Care work.

6. When I set my mind to it, I can learn everything that will be required when Trauma-Informed Care is adopted.

7. My past experiences make me confident that I will be able to perform successfully after Trauma-Informed Care is adopted.

Beliefs About Trauma and its Impact. Subheadings did not appear in Qualtrics. Answer choices $1=$ =strongly disagree, $2=$ moderately disagree, $3=$ mildly disagree, $4=$ not sure, $5=$ mildly agree, $6=$ moderately agree $7=$ strongly agree.

\section{Prevalence}

1. Many of the clients served by our agency have a history of psychological trauma.

2. Many of the staff in our agency have a history of psychological trauma.

\section{Trauma's impact on behavior and engagement}

3. Many problematic behaviors (such as substance abuse) start as a way to cope with 
emotionally traumatizing experiences.

4. Past experiences of psychological trauma (for instance in childhood) cannot be linked to current problematic behavior in adulthood. $\mathrm{R}$

5. When service recipients have experienced psychological trauma (current or in the past), this can influence their current behavior.

6. When staff have experienced psychological trauma (current or in the past), this can influence their current behavior at work.

\section{Service delivery and settings can be re-traumatizing}

7. Seeking and receiving services from our agency can be re-traumatizing for trauma survivors.

8. Our service setting does not create psychological trauma for our service recipients. $\mathrm{R}$

9. Our programs and services do not create psychological trauma for our service recipients. $\mathrm{R}$

10. Service providers can absorb the psychological trauma of service recipients.

11. Working with trauma survivors can result in work related stress such as vicarious trauma.

Organizational Strain: Subheadings did not appear in Qualtrics. Answer choices $1=$ strongly disagree, $2=$ moderately disagree, $3=$ =mildly disagree, $4=$ not sure, $5=$ mildly agree, $6=$ moderately agree $7=$ strongly agree.

\section{Stress}


1. The heavy staff workload reduces the effectiveness of my program.

2. I am under too many pressures to do my job effectively.

3. Staff members at my organization show signs of high stress and strain.

4. Staff frustration is common where I work.

\section{Turbulence}

5. This change toward Trauma-Informed Care is occurring during a turbulent time for our organization.

6. This change toward Trauma-Informed Care suffers from too many other distractions.

7. We were still trying to digest earlier changes when we embarked on TraumaInformed Care.

8. This change toward Trauma-Informed Care would have been easier if we were not already dealing with a number of other changes.

Previous Training. Answer choices 1=None, 2=Very little, 3=Some, 4=A lot.

1. How much face-to-face training have you had on the topics of trauma and TraumaInformed Care?

2. How much exposure through webinars have you had on the topics of trauma and Trauma-Informed Care?

3. How much exposure through books and other written materials have you had on the topics of trauma and Trauma-Informed Care? 
4. How much training have you had from a Trauma Informed Oregon trainer on the topics of trauma and Trauma-Informed Care?

Recency of Training. Answer choices $1=$ More than one year, $2=7-12$ months, 3= 2-6 months, $4=<1$ month.

1. How long has it been since your last training on the topics of trauma and TraumaInformed Care?

Work Experience. Answer choices $1=<2$ years, $2=2-5$ years, 3= 6-10 years, $4=$ More than 10 years.

1. How many years have you worked at your current organization?

2. How many years of experience do you have in your current field?

3. How many years have you been in the workforce (in any field)?

Demographic Information. These questions were either fill in the blank or multiple choice.

1. What field do you represent (pick the best choice, or select other)?

Physical health

Mental health

Substance abuse treatment

Public health

Child welfare
Criminal justice or law enforcement Education Early childhood

Other

2. What is your role?

Direct services (I work directly with service recipients / clients) 
Administrative or support

Management / Supervisory

Other

3. If you work for a specific program, what is this program? (e.g. Nurse Family Partnership, WIC, Self Sufficiency, Family Planning etc.)

4. What is your age?

5. What is your gender? 


\section{Appendix C}

\section{Descriptive Statistics}

\section{Table C1.}

Mean Values and Range for All Variables in Model

\begin{tabular}{lllrll}
\hline & $\begin{array}{l}\text { Missing } \\
(\%)\end{array}$ & Mean & $\begin{array}{l}\text { Std } \\
\text { Dev }\end{array}$ & Min & Max \\
\hline Affective & $0.0 \%$ & 6.28 & 1.29 & 1.17 & 7.00 \\
Beliefs $^{\mathrm{a}}$ & $5.2 \%$ & 5.92 & .71 & 2.60 & 7.00 \\
$\quad$ Beliefs - Prev & $4.2 \%$ & 6.14 & 1.09 & 1.00 & 7.00 \\
Beliefs - Imp & $5.7 \%$ & 6.60 & .58 & 3.75 & 7.00 \\
$\quad$ Beliefs - Svs & $5.1 \%$ & 5.11 & 1.02 & 1.40 & 7.00 \\
Self-Efficacy & $1.2 \%$ & 5.46 & 1.12 & 1.00 & 7.00 \\
Principal Support & $0.8 \%$ & 5.13 & 1.17 & 1.17 & 7.00 \\
Organizational & $6.5 \%$ & 5.09 & 1.02 & 1.13 & 7.00 \\
strain $^{\mathrm{b}}$ & & & & & \\
$\quad$ Stress & $5.9 \%$ & 5.29 & 1.34 & 1.00 & 7.00 \\
$\quad$ Turbulence $_{\text {Knowledge }}^{\mathrm{c}}$ & $7.0 \%$ & 4.84 & 1.24 & 1.00 & 7.00 \\
Known & $8.0 \%$ & 3.22 & .51 & 1.57 & 3.97 \\
\hline
\end{tabular}

Note. These values are reported at the item level in order to compare across scales.

${ }^{a}$ Beliefs is the observed variable resulting from factor analysis. This variable contains 10 of the items rather than 11 (see factor analysis section). ${ }^{\mathrm{b}}$ OrgStrain is the observed variable resulting from factor analysis and is a combination of stress and turbulence. ${ }^{c}$ Knowledge was measured using 4-point Likert scale. All other measures used a 7-point Likert scale. 


\section{Table C2.}

\section{Indicators of Normality - Skew and Kurtosis}

\begin{tabular}{lcccccc}
\hline Variable & Skew & SE & $Z_{\text {Skew }}$ & kurtosis & SE & $Z_{\text {kurt }}$ \\
\hline Affective & -2.541 & .223 & $-11.39^{* * *}$ & 6.229 & .442 & $14.093^{* * *}$ \\
Knowledge & -.874 & .243 & $-3.60^{* * *}$ & .703 & .481 & 1.46 \\
Beliefs $^{\mathrm{a}}$ & -1.910 & .231 & $-8.27^{* * *}$ & 6.305 & .459 & $13.74^{* * *}$ \\
$\quad$ Beliefs - & -2.685 & .227 & $-11.83^{* * *}$ & 9.156 & .451 & $20.30^{* * *}$ \\
Prev & & & & & & \\
$\quad$ Beliefs - & -2.299 & .231 & $-9.95^{* * *}$ & 6.443 & .459 & $14.04^{* * *}$ \\
$\quad$ Imp & & & & & & \\
$\quad$ Beliefs - & -.520 & .228 & $-2.28^{*}$ & .424 & .453 & .94 \\
Svs & & & $-5.91^{* * *}$ & 2.627 & .449 & $5.85^{* * *}$ \\
SelfEff & -1.336 & .226 & $-4.56^{* * *}$ & 1.139 & .444 & $2.57^{*}$ \\
PrinSupp & -1.022 & .224 & $-3.05^{* *}$ & 1.070 & .459 & $2.33^{*}$ \\
OrgStrain & -.705 & .231 & $-3.56^{* * *}$ & .318 & .455 & .70 \\
$\quad$ Stress & -.816 & .229 & -1.72 & -.219 & .459 & -.48 \\
$\quad$ Turb & -.398 & .231 & & & \\
\hline
\end{tabular}

Note. $\mathrm{SE}=$ standard error. Significance is based on $\mathrm{Z}$ score distribution.

${ }^{a}$ Beliefs is the observed variable resulting from factor analysis. This variable contains 10 of the items rather than 11 (see factor analysis section). ${ }^{b}$ Beliefs about service settings and programs includes the orginal 5 items. ${ }^{c}$ OrgStrain is the observed variable resulting from factor analysis and is a combination of stress and turbulence.

$* p<.05 * * p<.01 \quad * * * p<.001$ 


\section{Table C3.}

Tolerance and Variance Inflation Factor (VIF) for All Measures

\begin{tabular}{lcc}
\hline & Tolerance & VIF \\
\hline Knowledge & .644 & 1.552 \\
Beliefs $^{\mathrm{a}}$ & .567 & 1.764 \\
Self-Efficacy & .481 & 2.080 \\
Principal Support & .752 & 1.330 \\
Stress & .648 & 1.543 \\
Turbulence & .797 & 1.255 \\
\hline
\end{tabular}

Note: Dependent variable $=$ affective commitment. Because this analysis is based on linear regression, the composite organizational strain (OrgStrain) was left out of the model and the proposed measures of stress and turbulence were included.

${ }^{a}$ Beliefs is the observed variable resulting from factor analysis. This variable contains 10 of the items rather than 11 (see Developing Composite Variables section in Chapter 5). 
Table C4.

Analysis of Variance Based on Years of Experience

\begin{tabular}{ccccc}
\hline & M & SD & $F(\mathrm{df})$ & $p$ \\
\hline Knowledge & & & $.174(2,95)$ & .841 \\
Yrs Exp_High & 95.76 & 16.99 & & \\
Yrs Exp_Med & 97.77 & 14.95 & & \\
Yrs Exp_Low & 97.47 & 12.98 & & .398 \\
SelfEff & & & $.931(2,101)$ & \\
Yrs Exp_High & 36.90 & 8.36 & & \\
Yrs Exp_Med & 38.68 & 9.00 & & .189 \\
Yrs Exp_Low & 39.26 & 6.01 & & \\
& & & & \\
Beliefs & & & & \\
Yrs Exp_High & 58.76 & 8.07 & & \\
Yrs Exp_Med & 59.00 & 7.62 & & \\
Yrs Exp_Low & 59.97 & 5.44 & & \\
PrinSupp & & & & \\
Yrs Exp_High & 30.51 & 6.50 & & \\
Yrs Exp_Med & 29.86 & 7.77 & & \\
Yrs Exp_Low & 32.69 & 5.82 & & \\
& & & & \\
Affective Commitment & & & & \\
Yrs Exp_High & 36.07 & 9.18 & & \\
Yrs Exp_Med & 37.83 & 8.88 & & \\
Yrs Exp_Low & 40.25 & 2.97 & & \\
\hline
\end{tabular}

Note: Means are scale scores.

a Levene's test of homogeneity of variance was significant. Brown-Forsythe statistic is reported. 
Table C5.

Independent Samples t-Test Based on Prior Training

\begin{tabular}{llcccc}
\hline \multirow{2}{*}{ Knowledge } & M & SD & $t$ (df) & $p$ \\
& Training_high & 87.31 & 13.98 & $-8.079(80)$ & .00 \\
& Training_low & 106.25 & 8.51 & & \\
SelfEff & Training_high & 34.24 & 8.60 & $-5.22(75)$ & .00 \\
& Training_low & 41.57 & 4.98 & & \\
Beliefs & Training_high & 57.14 & 8.42 & $-3.140(101)$ & .00 \\
& Training_low & 61.33 & 4.79 & & \\
PrinSupp & Training_high & 29.88 & 7.61 & $-1.63(90)$ & .107 \\
& Training_low & 32.00 & 5.46 & & \\
Affective & Training_high & 35.35 & 10.08 & $-3.473(59)$ & .00 \\
Commitment & Training_low & 40.39 & 2.90 & &
\end{tabular}

Note: Means are scale scores. SelfEff $=$ self-efficacy. PrinSupp $=$ principal support. Df $=$ degrees of freedom.

a Levene's test of homogeneity of variance was significant; therefore, equal variance was not assumed. 


\section{Appendix D}

\section{Exploratory Factor Analysis}

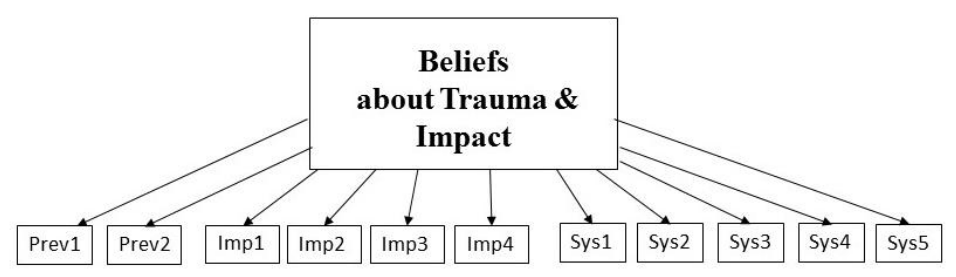

Figure D1. Observed variable beliefs

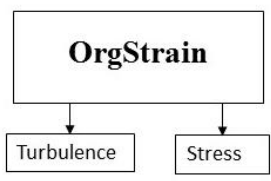

Figure D2. Observed variable organizational strain

Table D1.

Correlation Matrix of Items Measuring Beliefs

\begin{tabular}{|c|c|c|c|c|c|c|c|c|c|c|c|}
\hline & P1 & $\mathrm{P} 2$ & I1 & I2 & I3 & I4 & S1 & $\mathrm{S} 2$ & S3 & $\mathrm{S} 4$ & S5 \\
\hline $\mathrm{P} 1$ & 1.00 & .644 & .488 & .382 & .364 & .471 & .347 & .142 & .073 & .195 & .546 \\
\hline $\mathrm{P} 2$ & & 1.00 & $.467^{* *}$ & .198 & 196. & .433 & .381 & .352 & .343 & .100 & .470 \\
\hline I1 & & & 1.00 & .382 & .382 & .435 & .256 & .150 & .121 & .114 & .413 \\
\hline $\mathrm{I} 2$ & & & & 1.00 & .341 & .298 & .104 & .113 & .025 & .062 & .387 \\
\hline I3 & & & & & 1.00 & .386 & .055 & -.095 & -.129 & .095 & .370 \\
\hline I4 & & & & & & 1.00 & .292 & .211 & .197 & .104 & .547 \\
\hline $\mathrm{S} 1$ & & & & & & & 1.00 & .497 & .401 & .170 & .414 \\
\hline $\mathrm{S} 2$ & & & & & & & & 1.00 & .803 & .008 & .283 \\
\hline S3 & & & & & & & & & 1.00 & .001 & .286 \\
\hline S4 & & & & & & & & & & 1.00 & .269 \\
\hline S5 & & & & & & & & & & & 1.00 \\
\hline
\end{tabular}

Note: $\mathrm{P}=$ beliefs prevalence $\mathrm{I}=$ beliefs impact $\mathrm{S}=$ beliefs service systems 
Table D2.

Pattern Matrix for Beliefs Scale

\begin{tabular}{|c|c|c|c|}
\hline & $\begin{array}{c}\text { Comp } \\
1\end{array}$ & Comp 2 & $\begin{array}{c}\text { Comp } \\
3\end{array}$ \\
\hline $\begin{array}{l}\text { I3: When service recipients have experienced psychological } \\
\text { trauma (current or in the past), this can influence behavior. }\end{array}$ & .734 & -.330 & \\
\hline $\begin{array}{l}\text { I1: Many problematic behaviors (such as substance abuse) } \\
\text { start as a way to cope with traumatizing experiences. }\end{array}$ & .732 & & \\
\hline $\begin{array}{l}\text { P1: Many of the clients served by our agency have } \\
\text { experienced psychological trauma. }\end{array}$ & .728 & & \\
\hline $\begin{array}{l}\text { I4: When staff have experienced psychological trauma } \\
\text { (current or in the past), this can influence their current } \\
\text { behavior at work. }\end{array}$ & .681 & & \\
\hline $\begin{array}{l}\text { I2: Past experiences of psychological trauma (for instance in } \\
\text { childhood) cannot be linked to current problematic behaviors } \\
\text { in adulthood. }\end{array}$ & .679 & & \\
\hline $\begin{array}{l}\text { S5: Working with trauma survivors can result in work-related } \\
\text { stress such as vicarious trauma. }\end{array}$ & .614 & & \\
\hline $\begin{array}{l}\text { P2: Many of the staff in our agency have experienced } \\
\text { psychological trauma. }\end{array}$ & .547 & .392 & \\
\hline $\begin{array}{l}\text { S2: Our service setting does not create psychological trauma } \\
\text { for our service recipients. }\end{array}$ & & .925 & \\
\hline $\begin{array}{l}\text { S3: Our programs and services do not create psychological } \\
\text { trauma for our service recipients. }\end{array}$ & & .923 & \\
\hline $\begin{array}{l}\text { S1: Seeking and receiving services from our agency can be re- } \\
\text { traumatizing for trauma survivors. }\end{array}$ & & .588 & \\
\hline $\begin{array}{l}\text { S4: Service providers can absorb the psychological trauma of } \\
\text { service recipients. }\end{array}$ & & & .960 \\
\hline
\end{tabular}


Table D3.

Correlation Matrix of Items Measuring Organizational Strain

\begin{tabular}{lcccccccc}
\hline & Stress & Stress & Stress & Stress & Turb & Turb & Turb & Turb \\
& 1 & 2 & 3 & 4 & 1 & 2 & 3 & 4 \\
\hline Stress1 & 1.00 & .634 & .491 & .324 & .021 & .157 & .003 & .103 \\
Stress2 & & 1.00 & .598 & .483 & .124 & .112 & .135 & .257 \\
Stress3 & & & 1.00 & .700 & .298 & .131 & .218 & .201 \\
Stress4 & & & & 1.00 & .396 & .198 & .233 & .211 \\
Turbulence 1 & & & & 1.00 & .367 & .512 & .437 \\
Turbulence 2 & & & & & 1.00 & .413 & .366 \\
Turbulence 3 & & & & & & & 1.00 & .688 \\
Turbulence 4 & & & & & & & & 1.00 \\
\hline
\end{tabular}


Table D4.

Pattern Matrix for Organizational Strain

\begin{tabular}{|c|c|c|}
\hline & $\begin{array}{c}\text { Component } \\
1\end{array}$ & $\begin{array}{c}\text { Component } \\
2\end{array}$ \\
\hline $\begin{array}{l}\text { Stress2: I am under too many pressures to do my } \\
\text { job effectively. }\end{array}$ & .846 & \\
\hline $\begin{array}{l}\text { Stress3: Staff members at my agency show signs } \\
\text { of high stress and strain. }\end{array}$ & .840 & \\
\hline $\begin{array}{l}\text { Stress1: The heavy staff workload reduces the } \\
\text { effectiveness of my program. }\end{array}$ & .785 & \\
\hline Stress4: Staff frustration is common where I work. & .720 & .300 \\
\hline $\begin{array}{l}\text { Turbulence } 3 \text { : We were still trying to digest earlier } \\
\text { changes when we embarked on Trauma-Informed } \\
\text { Care. }\end{array}$ & & .867 \\
\hline $\begin{array}{l}\text { Turbulence 4: This change toward Trauma- } \\
\text { Informed Care would have been easier if we were } \\
\text { not already dealing with a number of changes. }\end{array}$ & & .799 \\
\hline $\begin{array}{l}\text { Turbulence 1: This change toward Trauma- } \\
\text { Informed Care is occurring during a turbulent time } \\
\text { for our organization. }\end{array}$ & & .744 \\
\hline $\begin{array}{l}\text { Turbulence } 2 \text { : This change toward Trauma- } \\
\text { Informed Care suffers from too many other } \\
\text { distractions. }\end{array}$ & & .635 \\
\hline
\end{tabular}

Note: Initial principal components analysis with orthogonal (varimax) rotation. 